\title{
Expansion of Einstein-Yang-Mills theory by differential operators
}

\author{
Bo Feng $\odot,{ }^{1,2, \dagger}$ Xiaodi Li $\odot,{ }^{1, *}$ and Kang Zhou $\odot^{3, *}$ \\ ${ }^{1}$ Zhejiang Institute of Modern Physics, Zhejiang University, Hangzhou 310027, P. R. China \\ ${ }^{2}$ Center of Mathematical Science, Zhejiang University, Hangzhou 310027, P. R. China \\ ${ }^{3}$ Center for Gravitation and Cosmology, College of Physical Science and Technology, \\ Yangzhou University, Yangzhou 225009, P.R. China
}

(Received 28 July 2019; published 11 December 2019)

\begin{abstract}
The factorization form of the integrands in the Cachazo-He-Yuan formalism makes the generalized Kawai-Lewellen-Tye relations manifest; thus, amplitudes of one theory can be expanded in terms of the amplitudes of another theory. Although this claim seems a rather natural consequence of the above structure, finding the exact expansion coefficients to express an amplitude in terms of another amplitudes is, nonetheless, a nontrivial task despite many efforts devoted to it in the literature. In this paper, we propose a new strategy based on using the differential operators introduced by Cheung, Shen, and Wen, and taking advantage of the fact that these operators already relate the amplitudes of different theories. Using this new method, expansion coefficients can be found effectively. Although the method should be general, to demonstrate the idea, we focus on the expansion of single trace Einstein-Yang-Mills (sEYM) amplitudes in the Kleiss-Kuijf basis and Bern-Carrasco-Johansson (BCJ) basis of Yang-Mills theory. Using the new method, the general recursive expansion to the Kleiss-Kuijf basis has been reproduced. The expansion to the BCJ basis is a more difficult problem. Using the new method, we have worked out the details for sEYM with one, two, and three gravitons. As a by-product, profound relations among two kinds of expansion coefficients, i.e., the expansion of sEYM amplitudes to the BCJ basis of Yang-Mills theory and the expansion of any color ordered Yang-Mills amplitudes to its BCJ basis, have been observed.
\end{abstract}

DOI: $10.1103 /$ PhysRevD.100.125012

\section{INTRODUCTION}

Witten's famous work [1] has opened a door to study scattering amplitudes from angles different from the standard Feynman diagrams. One of such new angles is the CachazoHe-Yuan (CHY) formalism [2-6]. The CHY formalism has made many beautiful properties manifest, such as the gauge invariance, the soft behavior [7-14], the double copy structure $[15,16]$, etc. Among those important results, two of them are related to our current work. The first one is that for all theories known now in the CHY formalism, the weight-four $\mathrm{CHY}$ integrands can be factorized as the product of two weight-two ingredients, formally written as

$$
\mathcal{I}=\mathcal{I}_{L} \times \mathcal{I}_{R}
$$

\footnotetext{
*Corresponding author. zhoukang@yzu.edu.cn.

fengbo@zju.edu.cn

"lixiaodi@zju.edu.cn

Published by the American Physical Society under the terms of the Creative Commons Attribution 4.0 International license. Further distribution of this work must maintain attribution to the author(s) and the published article's title, journal citation, and DOI. Funded by SCOAP ${ }^{3}$.
}

For each weight-two ingredient, when multiplying by another particular weight-two ingredient, i.e., the color ordered Parke-Taylor factor,

$$
\operatorname{PT}(\alpha):=\frac{1}{\left(z_{\alpha_{1}}-z_{\alpha_{2}}\right) \cdots\left(z_{\alpha_{n-1}}-z_{\alpha_{n}}\right)\left(z_{\alpha_{n}}-z_{\alpha_{1}}\right)},
$$

they define corresponding color ordered amplitudes $A_{L}(\alpha), A_{R}(\beta)$. Thus (1.1) naturally gives the well known generalized Kawai-Lewellen-Tye (KLT) [17] relations (or the double copy form), ${ }^{1}$

$$
A=\sum_{\sigma, \tilde{\sigma} \in S_{n-3}} A_{L}(n-1, n, \sigma, 1) \mathcal{S}[\sigma \mid \tilde{\sigma}] A_{R}(1, \tilde{\sigma}, n-1, n) .
$$

As pointed out in [19], the double copy form implies immediately that the original amplitude $A$ can be expanded in terms of either the amplitudes $A_{L}$ or the amplitudes $A_{R}$. For example, with a fixed $\tilde{\sigma}$ ordering, we sum $\sigma$ over all permutations of $S_{n-3}$ and denote the result as

\footnotetext{
${ }^{1}$ See Sec. 5 of [18] for a comprehensive up-to-date list of the theories connected by double-copy relations.
} 


$$
\mathcal{C}(\tilde{\sigma}):=\sum_{\sigma \in S_{n-3}} A_{L}(n-1, n, \sigma, 1) \mathcal{S}[\sigma \mid \tilde{\sigma}]
$$

then the original amplitude can be rewritten as

$$
A=\sum_{\tilde{\sigma} \in S_{n-3}} \mathcal{C}(\tilde{\sigma}) A_{R}(1, \tilde{\sigma}, n-1, n),
$$

where $\mathcal{C}(\tilde{\sigma})$ 's serve as the expansion coefficients. Above, short deduction seems to indicate that expanding amplitudes of one theory in terms of amplitudes of another theory is a trivial consequence of the double copy form (1.3). However, such a triviality is just an illusion. When trying to get the exact expansion coefficients $\mathcal{C}$, one will find it is very difficult task. If we use the expression (1.4) directly, one needs to do the sum over $(n-3)$ ! terms. Since analytical expressions for both parts $A_{L}(n-1$, $n, \sigma, 1)$ and $\mathcal{S}[\sigma \mid \tilde{\sigma}]$ are very complicated, it is no wonder why, in practice, no one uses this method except for in some special cases. ${ }^{2}$ Much effort has been devoted to avoid the mentioned technical difficulties. In [21], using the string theory, the single trace Einstein-Yang-Mills (sEYM) amplitudes with just one graviton have been expanded in terms of the color ordered Yang-Mills (YM) amplitudes. In [22], using the heterotic string theory, evidence of the expansion of general EYM amplitudes in terms of YM amplitudes has also been presented, but explicit expansions are done only for some special cases, such as up to three gravitons, or up to two color traces. In [23], using the fact that expansion coefficients $\mathcal{C}$ 's in the Kleiss-Kuijf (KK) basis can be identified with the BCJ numerators of the DelDuca-Dixon-Maltoni (DDM) chain [24], an assumption of algebraic expressions has been made with the proper hints from Feynman diagrams of the DDM chain. After that, the gauge invariance and other properties have been imposed on the assumption; thus, expansion coefficients up to five gravitons have been finally determined. In $[25,26]$, using the CHY integrand and the techniques developed and presented in [27-30], such as the cross ratio identities etc., expansion coefficients of sEYM amplitudes up to four gravitons have been presented. In these works, using the CHY integrands instead of amplitudes directly is a big simplification, since the analytical expressions of CHY integrands are much easier to write down while analytical expressions of amplitudes are very hard to imagine. However, since the cross ratio identities have a lot of equivalent forms, results obtained by this way contain a lot of freedoms; thus, it is very hard to observe the general patterns from these explicit examples. A different approach has been used in [19] to attack this difficult problem: with the

\footnotetext{
${ }^{2}$ In [20], since all amplitudes are the maximally helicity violating amplitudes, the analytical expressions are very simple, thus one can carry out the $(n-3)$ ! sum recursively.
}

proper arrangement of the known expansion coefficients for one, two, and three gravitons, gauge symmetry consideration has led us to a conjecture having the natural iterative expansion pattern. Thus, the expansion coefficients for an arbitrary number of gravitons in the sEYM theory can be systematically worked out. With the guidance of the conjectured pattern in [19], rigorous proof of the conjecture has been given in [31] by carefully dealing with the CHY integrands of sEYM theory, especially the Pfaffian structure. Based on these results, expansions for multitrace EYM amplitudes as well as nonlinear sigma model amplitudes have been given in $[32,33]$, again using the gauge symmetry considerations plus the manipulations of the CHY integrands.

The second important result coming from the $\mathrm{CHY}$ formalism is that different theories have some deep connections, where KLT-like relations are just a reflection of them. In [6], using three operations, i.e., dimension reduction, squeezing, and generalized dimension reduction, various theories can be deduced from the Einstein gravity theory. Recently, the web of connections of different theories given in [6] have also been reproduced in [34] from a different point of view. It is found that one can define different kinds of differential operators, which act on variables formed by Lorentz contractions among external momenta and polarization vectors. When the proper combinations of differential operators act on the on-shell amplitudes of one theory, amplitudes of another theory will be reached. Since these two different strategies lead to the same web picture, there must be a connection between them. Such a connection has been explained in $[35,36]$, by showing that when acting proper combinations of differential operators on the CHY integrands of Einstein gravity theory, the corresponding $\mathrm{CHY}$ integrands of other theories have been produced.

The above two results lead naturally to the following question: since, by using differential operators, one can connect different theories, could we use them to establish expansion relations as given in (1.5)? In this paper, we will show that indeed one can do that. The basic idea is the following: by applying differential operators at the both sides of (1.5), one gets differential equations for expansion coefficients. Solving them, one obtains the wanted coefficients. In fact, we achieve more than just finding coefficients. We will show that by proper choices of building blocks, we can derive the expansion relation (1.5) and find coefficients at the same time. More explicitly, when we act on differential operators on amplitudes of sEYM theory at the left-hand side, we land naturally to YM amplitudes at the right-hand side. The emergence of relations (1.5) comes from the web connections between different theories through differential operators as explained in [34].

Although our new method is general, in this paper, we will mostly focus on the expansion of sEYM amplitudes. The reason is that as shown in $[6,34]$, by acting differential 
operators on amplitudes of the sEYM theory, ${ }^{3}$ one can get amplitudes of all other theories in the web (see also $[35,36])$. Thus, if we can solve the expansion of sEYM amplitudes using the new method, such a method should be applicable to all other theories. Furthermore, when we talk about the expansion, we need to be careful about two different kinds of expansions. The factorization form of CHY integrands leads naturally to the expansion form (1.5), where the independent basis is $(n-3)$ ! color ordered amplitudes with three legs fixed. This basis is called the Bern-Carrasco-Johansson (BCJ) basis. As we will show, with different choices of building blocks, our new method will give either the expansion to the $\mathrm{BCJ}$ basis, or the expansion to the KK basis, which has $(n-2)$ ! color ordered amplitudes with only two legs fixed (for example, the first and the last legs). Results reviewed in the above references (such as $[19,21-23,25,26,31-33]$ ) are mostly expanded in the KK basis. Although using the BCJ relations among KK basis [15,37-40] one can connect these two kinds of expansions; such a connection is not so easy to get as we will show in this paper. Another motivation of our current paper is to expand sEYM amplitudes directly in the BCJ basis without relying on expansion in the KK basis.

Having the above clarifications, the tasks of the paper are the following two: (1) First we rederive expansions in the KK basis using only differential operators; (2) second, we derive expansion in the $\mathrm{BCJ}$ basis directly. Our derivations will deepen our understanding of the intricate connections between on-shell amplitudes of different theories. For example, we will see how the expansion coefficients of sEYM amplitudes in (1.5) and the expansion coefficients [see (2.14)]) of arbitrary color ordered YM amplitudes in its $\mathrm{BCJ}$ basis are related to each other.

The plan of the paper is the following. In Sec. II, we present some backgrounds needed for later discussions. They include the expansion of arbitrary color ordered YM amplitudes in its BCJ basis, the recursive expansion of sEYM amplitudes in the KK basis of YM amplitudes, and the differential operators used heavily in this paper. In Sec. III, we rederive the recursive expansion of sEYM amplitudes in the KK basis of YM amplitudes using the proposed new method, i.e., using differential operators. As emphasized in previous paragraphs, results in this section show that the differential operator method is an independent systematical new method to determine the expansion. In Sec. IV, we apply the same method to Einstein gravity theory and Born-Infeld theory. In Sec. V, starting from the expansion of sEYM amplitudes in the KK basis of YM amplitudes, with careful algebraic manipulations, we translate the $\mathrm{KK}$ basis to the $\mathrm{BCJ}$ basis and thus get the

\footnotetext{
${ }^{3}$ The CHY integrand of gravity theory is defined by the Pfaffian of a matrix, which, when expanding, becomes the sum of integrands of sEYM theory with different gluon structures.
}

expansion in the BCJ basis. In Sec. VI, following the similar idea of Sec. III but with a different choice of building blocks, we derive the expansion of sEYM amplitudes in the BCJ basis with one, two, and three gravitons by applying differential operators directly. In Sec. VII, some discussions have been given. Some technical calculations are collected in the Appendixes. In Appendix A, we show that terms with an index cycle structure will drop out from the expansion. In Appendix B, we discuss the structure of functions when imposing manifestly gauge invariant conditions. These structures provide the building blocks used in the expansion in the BCJ basis. In Appendix C, some omitted calculation details in Sec. VI have been given.

\section{SOME BACKGROUNDS}

In this section, we will review some known results. They will serve as the backgrounds of our discussions in the whole paper. This section contains three parts. In the first part, we review the expansion of arbitrary color ordering YM amplitudes to its BCJ basis. Especially, we have rewritten the expansion coefficients in a compact form comparing to thOse given in $[15,38]$. In the second part, we review the recursive expansion of SEYM amplitudes in the KK basis of YM amplitudes given in $[19,31,33]$. The result will be reproduced by using differential operators proposed in this paper. In the third part, we review differential operators introduced in [34]. They are our main tools. As a simple application of these tools, three simple but nontrivial new relations for EYM amplitudes have been derived in (2.31), (2.37), and (2.38).

\section{A. The expansion of color ordered YM amplitudes to its BCJ basis}

It is well known that all color ordered Yang-Mills amplitudes are not independent of each other. There are two kinds of relations among them. The first kind is the KK relation [41], which is given by

$$
A(1, \alpha, n, \beta)=\sum_{ш}(-)^{n_{\beta}} A\left(1, \alpha ш \beta^{T}, n\right)
$$

where $\alpha, \beta$ are two ordered subsets of external gluons and $n_{\beta}$ is the number of elements in the subset $\beta$, while $\beta^{T}$ means the reversing of the ordered subset $\beta$. The sum is over all possible shuffles of two ordered subsets. All shuffles can be obtained recursively as

$$
\begin{aligned}
& \alpha ш \varnothing=\alpha, \quad \varnothing ш \beta=\beta, \\
& \left\{\alpha_{1}, \ldots, \alpha_{m}\right\} ш\left\{\beta_{1}, \ldots, \beta_{k}\right\} \\
& \quad=\left\{\alpha_{1},\left\{\alpha_{2}, \ldots, \alpha_{m}\right\} ш \beta\right\}+\left\{\beta_{1}, \alpha ш\left\{\beta_{2}, \ldots, \beta_{k}\right\}\right\} .
\end{aligned}
$$


Using the KK relations (2.1), one can expand arbitrary color ordered Yang-Mills amplitudes in the basis, where two legs are fixed at the two ends (hence, we will call such a basis the "KK basis").

The second kind are the BCJ relations [15]. To present these relations compactly, let us define some notations first. Given two ordered sets $\Xi=\left\{\xi_{1}, \xi_{2}, \ldots, \xi_{n}\right\}$ and $\beta=$ $\left\{\beta_{1}, \ldots, \beta_{r}\right\}$ where the set $\beta$ is the subset of $\Xi$, for a given element $p \in \Xi$ with its position $K$ in $\Xi$ (i.e., $\xi_{K}=p$ ), we define

(1) The momentum $X_{p}$ : It is given by

$$
X_{p}=\sum_{i=1}^{K-1} k_{\xi_{i}}
$$

i.e., the sum of momenta of these elements, which are on the left-hand side of $p$ in the set $\Xi$.

(2) The momentum $Y_{p}$ : It is given by

$$
Y_{p}=\sum_{i=1, \xi_{i} \notin \beta}^{K-1} k_{\xi_{i}},
$$

i.e., the sum of momenta of these elements, which are on the left-hand side of $p$ in the set $\Xi$, but do not belong to the set $\beta$.

(3) The momenta $W_{p}^{(L, L)}, W_{p}^{(L, R)}, W_{p}^{(R, L)}, W_{p}^{(R, R)}$ : To define them, we require $p \in \beta$, thus $p$ split the set $\beta$ into two subsets $\beta_{p}^{L}$ and $\beta_{p}^{R}$, i.e., the collections of elements on the left-hand side of $p$ and on the righthand side of $p$, respectively. Now we define

$$
W_{p}^{(L, L)}=\sum_{i=1, \xi \notin \beta_{p}^{R}}^{K-1} k_{\xi_{i}},
$$

i.e., $W_{p}^{(L, L)}$ is the sum of momenta of these elements, which are on the left-hand side of $p$ in the set $\Xi$, but do not belong to the subset $\beta_{p}^{R}$. In other words, $W_{p}^{(L, L)}$ is the sum of momenta of these elements at the left-hand side of $p$ in the set $\Xi$ satisfying either the condition that they do not belong to the set $\beta$ or the condition that they belong to the subset $\beta_{p}^{L}$. This is the reason we call it $W_{p}^{(L, L)}$, where the first $L$ is for these elements belonging to $\Xi \backslash \beta$, and the second $L$ is for these elements belonging to $\beta_{p}^{L}$. Similarly, we define

$$
\begin{aligned}
W_{p}^{(L, R)} & =\sum_{i=1, \xi \notin \beta_{p}^{L}}^{K-1} k_{\xi_{i}}, \\
W_{p}^{(R, L)} & =\sum_{i=K+1, \xi \notin \beta_{p}^{R}}^{n} k_{\xi_{i}}, \\
W_{p}^{(R, R)} & =\sum_{i=K+1, \xi \notin \beta_{p}^{L}}^{n} k_{\xi_{i}} .
\end{aligned}
$$

After the above preparations, let us begin with the (generalized) fundamental BCJ relation [38]. If we divide the set $\{2, \ldots, n-1\}$ into two ordered subsets $\alpha=$ $\left\{a_{1}, \ldots, \alpha_{m}\right\}$ and $\beta=\left\{\beta_{1}, \ldots, \beta_{t}\right\}$ (so $m+t=n-2$ ), the relations are given by

$$
\sum_{\Psi}\left(\sum_{i=1}^{t} k_{\beta_{i}} \cdot X_{\beta_{i}}\right) A(1, \alpha \omega \beta, n)=0 .
$$

Using these relations, one can easily solve

$$
A_{n+1}^{\mathrm{YM}}(1, p,\{2, \ldots, n-1\}, n)=\frac{-\left(k_{p} \cdot X_{p}\right)}{\left(k_{p} \cdot k_{1}\right)} A_{n+1}^{\mathrm{YM}}(1,2,\{3, \ldots, n-1\} ш\{p\}, n)
$$

for $\Xi=\{1,\{2, \ldots, n-1\} ш\{p\}, n\}$ and $\beta=\{p\}$, and

$$
\begin{aligned}
A_{n+2}^{\mathrm{YM}}(1, p, q,\{2, \ldots, n-1\}, n)= & \frac{\left(k_{p} \cdot k_{1}+k_{q} \cdot\left(Y_{q}+k_{p}\right)\right)\left(k_{p} \cdot\left(Y_{p}+k_{q}\right)\right)}{\mathcal{K}_{1 p q} \mathcal{K}_{1 p}} A_{n+1}^{\mathrm{YM}}(1,2,\{3, \ldots, n-1\} ш\{q, p\}, n) \\
& +\frac{\left(k_{p} \cdot\left(Y_{p}-k_{1}\right)\right)\left(k_{q} \cdot\left(Y_{q}+k_{p}\right)\right)}{\mathcal{K}_{1 p q} \mathcal{K}_{1 p}} A_{n+2}^{\mathrm{YM}}(1,2,\{3, \ldots, n-1\} ш\{p, q\}, n)
\end{aligned}
$$

for $\Xi=\{1,\{2, \ldots, n-1\} ш\{p, q\}, n\}$ and $\beta=\{p, q\}$. For simplicity, we have omitted the $\sum_{\omega}$ on the right-hand side when its meaning is obvious and defined

$$
\mathcal{K}_{\alpha}=\sum_{i<j ; i, j \in \alpha} k_{i} \cdot k_{j}
$$

for any set $\alpha$. 
As shown in [38], by recursively using the relations (2.9), one can expand arbitrary color ordered Yang-Mills amplitudes in the BCJ basis, i.e., those color ordered YangMills amplitudes with three legs fixed at some particular positions (for example, at the first, second, and the last positions), as

$$
\begin{aligned}
& A_{n}\left(1, \beta_{1}, \ldots, \beta_{r}, 2, \alpha_{1}, \ldots, \alpha_{n-r-3}, n\right) \\
& \quad=\sum_{\{\xi\} \in\{\beta\} \sqcup \mathcal{P}\{\alpha\}} \mathcal{C}_{\{\alpha\},\{\beta\} ;\{\xi\}} A_{n}(1,2,\{\xi\}, n) .
\end{aligned}
$$

The expansion coefficients (we will call them the "BCJ coefficients") are first conjectured in [15] and then proved in [38]. Using our new defined notations, they are given by

$$
\mathcal{C}_{\{\alpha\},\{\beta\} ;\{\xi\}}=\prod_{k=1}^{r} \frac{\mathcal{F}_{\beta_{k}}(\{\alpha\},\{\beta\} ;\{\xi\})}{\mathcal{K}_{1 \beta_{1} \ldots \beta_{k}}},
$$

where

$$
\begin{aligned}
\mathcal{F}_{\beta_{k}}(\{\alpha\},\{\beta\} ;\{\xi\}) \\
=\theta\left(\xi_{\beta_{k}}-\xi_{k-1}\right)\left\{k_{\beta_{k}} \cdot W_{\beta_{k}}^{(R, R)}+\theta\left(\xi_{\beta_{k+1}}-\xi_{\beta_{k}}\right) \mathcal{K}_{1 \beta_{1} \ldots \beta_{k}}\right\} \\
\quad+\theta\left(\xi_{\beta_{k-1}}-\xi_{k}\right)\left\{-k_{\beta_{k}} \cdot\left(W_{\beta_{k}}^{(L, R)}-k_{1}\right)\right. \\
\left.\quad-\theta\left(\xi_{\beta_{k}}-\xi_{\beta_{k+1}}\right) \mathcal{K}_{1 \beta_{1} \ldots \beta_{k}}\right\}
\end{aligned}
$$

with $W$ 's defined in (2.8) and (2.6) and $\theta(x)=1$ when $x>0$ and $\theta(x)=0$, otherwise. ${ }^{4}$ Results (2.13), (2.14), and (2.15) are a little bit complicated and we give some explanations:

(i) In (2.13), we have defined a new notation ${w_{\mathcal{P}}}_{\text {}}$, the "partial ordered shuffle", which is the sum over all permutations that maintain only the relative order of the subset $\{\alpha\}$ (i.e., the ordering of elements of the subset $\{\beta\}$ in $\{\xi\}$ can be arbitrary).

(ii) The expression (2.14) tells us that for each element $\beta_{k} \in \beta$, there is an associated kinematic factor. The BCJ coefficients are the products of these factors.

(iii) For the set $\{\xi\}=\left\{\xi_{1}, \ldots, \xi_{n-3}\right\}$, we use the $\xi_{\beta_{k}}$ to denote the position of the element $\beta_{k}$ in $\xi$. Also, we define the auxiliary elements $\beta_{0}, \beta_{r+1}$ with positions $\xi_{\beta_{0}} \equiv+\infty$ and $\xi_{\beta_{r+1}}=0$; i.e., $\beta_{0}$ is always treated at the rightmost position and $\beta_{r+1}$ is always treated at the leftmost position. With this understanding, the $\theta(x)$ 's are well defined for all $\beta_{k}$ with $k=1,2, \ldots, r$.

Having explained the meanings of (2.13), (2.14), and (2.15), let us apply them to one example:

\footnotetext{
${ }^{4}$ It is worth emphasizing that in the definition of (2.15), there are three orderings $\{\alpha\},\{\beta\}$, and $\{\xi\}$. To define $W$ 's we need both of them, i.e., $\beta=\left\{\beta_{1}, \ldots, \beta_{r}\right\}$ and $\Xi=\{1,2, \xi, n\}$. To define $\theta(x)$ only $\{\xi\}$ is needed.
}

$$
\begin{aligned}
& A_{n+2}^{\mathrm{YM}}(1, p, q, r,\{2, \ldots, n-1\}, n) \\
& =\sum_{\rho \in S_{3}} \mathcal{C}[\{p, q, r\} ; \rho\{p, q, r\}] \\
& \quad \times A_{n+3}^{\mathrm{YM}}(1,2,\{3, \ldots, n-1\} ш\{\rho\{p, q, r\}\}, n)
\end{aligned}
$$

where the sum is over all permutations $\rho$ of three legs $\{p, q, r\}$. Coefficients in (2.16) involve two ordered lists: the first one $\{p, q, r\}$ is the remembering of the original ordering on the left-hand side, while the second one $\rho\{p, q, r\}$ is the ordering in the expansion on the righthand side. For these six coefficients with orderings $\rho\{p, q, r\}$, we have

(i) The order $\{p, q, r\}$ : For $p, \xi_{0}=+\infty>\xi_{p}$ and $\xi_{p}<\xi_{q}$, so we have

$$
-k_{p} \cdot\left(W_{p}^{(L, R)}-k_{1}\right)=-k_{p} \cdot\left(Y_{p}-k_{1}\right) .
$$

For $q, \xi_{p}<\xi_{q}$ and $\xi_{q}<\xi_{r}$, so we have

$$
\begin{aligned}
k_{q} \cdot W_{q}^{(R, R)}+\mathcal{K}_{1 p q} & =-k_{q} \cdot X_{q}+\mathcal{K}_{1 p q} \\
& =-k_{q} \cdot\left(Y_{q}+k_{p}\right)+\mathcal{K}_{1 p q}
\end{aligned}
$$

where momentum conservation has been used. For $r$, $\xi_{q}<\xi_{r}$ and $\xi_{r}>0$, so we have

$$
k_{r} \cdot W_{r}^{(R, R)}=-k_{r} \cdot X_{r}
$$

This ordering is a special case since it is the same ordering compared to the original ordering. This case is easy to generalize to a set with arbitrary length $m$ and we have the following results. For $\beta_{r}$, which is not the first or the last elements of $\beta$, the corresponding kinematic factor is

$$
k_{\beta_{r}} \cdot W_{\beta_{r}}^{(R, R)}+\mathcal{K}_{1 \beta_{1} \ldots \beta_{r}}=-\left(k_{\beta_{r}} \cdot X_{\beta_{r}}-\mathcal{K}_{1 \beta_{1} \ldots \beta_{r}}\right) .
$$

For the first element, the corresponding kinematic factor is

$$
-k_{\beta_{1}} \cdot\left(W_{\beta_{1}}^{(L, R)}-k_{1}\right)=-k_{\beta_{1}} \cdot\left(Y_{\beta_{1}}-k_{1}\right) .
$$

For the last element, the corresponding kinematic factor is

$$
k_{\beta_{m}} \cdot W_{\beta_{m}}^{(R, R)}=k_{\beta_{m}} \cdot \bar{Y}_{\beta_{m}}=-k_{\beta_{m}} \cdot X_{\beta_{m}} .
$$

(ii) The order $\{p, r, q\}$ : For $p, \xi_{0}=+\infty>\xi_{p}$ and $\xi_{p}<$ $\xi_{q}$, so we have $-k_{p} \cdot\left(W_{p}^{(L, R)}-k_{1}\right)=-k_{p} \cdot\left(Y_{p}-k_{1}\right)$. For $q, \quad \xi_{p}<\xi_{q}$ and $\xi_{q}>\xi_{r}$, so we have $k_{q} \cdot W_{q}^{(R, R)}=k_{q} \cdot \bar{Y}_{q}=-k_{q} \cdot X_{q}$. For $r, \xi_{q}>\xi_{r}$ 
and $\xi_{r}>0$, so we have $-k_{r} \cdot\left(W_{r}^{(L, R)}-k_{1}\right)-$ $\mathcal{K}_{1 p q r}=-k_{r} \cdot\left(Y_{r}-k_{1}\right)-\mathcal{K}_{1 p q r}$.

(iii) The order $\{q, p, r\}$ : For $p, \xi_{0}=+\infty>\xi_{p}$ and $\xi_{p}>\xi_{q}$, so we have $-k_{p} \cdot\left(W_{p}^{(L, R)}-k_{1}\right)-\mathcal{K}_{1 p}=$ $-k_{p} \cdot X_{p}$. For $q, \xi_{p}>\xi_{q}$ but $\xi_{q}<\xi_{r}$, so we have $-k_{q} \cdot\left(W_{q}^{(L, R)}-k_{1}\right)=-k_{q} \cdot\left(Y_{q}-k_{1}\right)$. For $r, \xi_{q}<$ $\xi_{r}$ and $\xi_{r}>0$, so we have $k_{r} \cdot W_{r}^{(R, R)}=-k_{r} \cdot X_{r}$.

(iv) The order $\{q, r, p\}$ : For $p, \xi_{0}=+\infty>\xi_{p}$ and $\xi_{p}>\xi_{q}$, so we have $-k_{p} \cdot\left(W_{p}^{(L, R)}-k_{1}\right)-\mathcal{K}_{1 p}=$ $-k_{p} \cdot X_{p}$. For $q, \xi_{p}>\xi_{q}$ and $\xi_{q}<\xi_{r}$, so we have $-k_{q} \cdot\left(W_{q}^{(L, R)}-k_{1}\right)=-k_{q} \cdot\left(Y_{q}-k_{1}\right)$. For $r, \xi_{q}<\xi_{r}$ and $\xi_{r}>0$, so we have $k_{r} \cdot W_{r}^{(R, R)}=-k_{r} \cdot\left(X_{r}+k_{p}\right)$.

(v) The order $\{r, p, q\}$ : For $p$, we have $\xi_{0}=+\infty>\xi_{p}$ and $\xi_{p}<\xi_{q}$, so we will have $-k_{p} \cdot\left(W_{p}^{(L, R)}-k_{1}\right)=$ $-k_{p} \cdot\left(X_{p}-k_{1}\right)$. For $q$, we have $\xi_{p}<\xi_{q}$ and $\xi_{q}>\xi_{r}$, so it is $k_{q} \cdot W_{q}^{(R, R)}=-k_{q} \cdot X_{q}$. For $r, \xi_{q}>\xi_{r}$ and $\xi_{r}>0, \quad$ so it is $-k_{r} \cdot\left(W_{r}^{(L, R)}-k_{1}\right)-\mathcal{K}_{1 p q r}=$ $-k_{r} \cdot\left(Y_{r}-k_{1}\right)-\mathcal{K}_{1 p q r}$.

(vi) The order $\{r, q, p\}$ : This case is also special, since the ordering $\{r, q, p\}$ is the reversing of the original one $\{p, q, r\}$. In general, for the ordering $\beta^{T}$, each element $\beta_{r}$ has the corresponding kinematic factor

$$
\begin{aligned}
& -k_{\beta_{r}} \cdot\left(W_{\beta_{r}}^{(L, R)}-k_{1}\right)-\mathcal{K}_{1 \beta_{1} \ldots \beta_{r}} \\
& =-k_{\beta_{r}} \cdot\left(X_{\beta_{r}}-k_{1}\right)-\mathcal{K}_{1 \beta_{1} \ldots \beta_{r}} .
\end{aligned}
$$

For later convenience, we collect these six coefficients as follows:

$$
\begin{aligned}
& \mathcal{C}[\{p, q, r\} ;\{p, q, r\}]=\frac{-\left(k_{p} \cdot\left(Y_{p}-k_{1}\right)\right)}{\mathcal{K}_{1 p}} \times \frac{\left(\mathcal{K}_{1 p q}-k_{q} \cdot X_{q}\right)}{\mathcal{K}_{1 p q}} \times \frac{-\left(k_{r} \cdot X_{r}\right)}{\mathcal{K}_{1 p q r}} \\
& \mathcal{C}[\{p, q, r\} ;\{p, r, q\}]=\frac{-\left(k_{p} \cdot\left(Y_{p}-k_{1}\right)\right)}{\mathcal{K}_{1 p}} \times \frac{-\left(k_{q} \cdot X_{q}\right)}{\mathcal{K}_{1 p q}} \times \frac{-k_{r} \cdot\left(Y_{r}-k_{1}\right)-\mathcal{K}_{1 p q r}}{\mathcal{K}_{1 p q r}} \\
& \mathcal{C}[\{p, q, r\} ;\{q, p, r\}]=\frac{-k_{p} \cdot X_{p}}{\mathcal{K}_{1 p}} \times \frac{-\left(k_{q} \cdot\left(Y_{q}-k_{1}\right)\right)}{\mathcal{K}_{1 p q}} \times \frac{-k_{r} \cdot X_{r}}{\mathcal{K}_{1 p q r}} \\
& \mathcal{C}[\{p, q, r\} ;\{q, r, p\}]=\frac{-k_{p} \cdot X_{p}}{\mathcal{K}_{1 p} \times \frac{-\left(k_{q} \cdot\left(Y_{q}-k_{1}\right)\right)}{\mathcal{K}_{1 p q}} \times \frac{-k_{r} \cdot\left(X_{r}+k_{p}\right)}{\mathcal{K}_{1 p q r}}} \\
& \mathcal{C}[\{p, q, r\} ;\{r, p, q\}]=\frac{-\left(k_{p} \cdot\left(X_{p}-k_{1}\right)\right)}{\mathcal{K}_{1 p}} \times \frac{-k_{q} \cdot X_{q}}{\mathcal{K}_{1 p q}} \times \frac{-k_{r} \cdot\left(Y_{r}-k_{1}\right)-\mathcal{K}_{1 p q r}}{\mathcal{K}_{1 p q r}} \\
& \mathcal{C}[\{p, q, r\} ;\{r, q, p\}]=\frac{-k_{p} \cdot X_{p}}{\mathcal{K}_{1 p}} \times \frac{-k_{q} \cdot\left(X_{q}-k_{1}\right)-\mathcal{K}_{1 p q}}{\mathcal{K}_{1 p q}} \times \frac{-k_{r} \cdot\left(Y_{r}-k_{1}\right)-\mathcal{K}_{1 p q r}}{\mathcal{K}_{1 p q r}} .
\end{aligned}
$$

Before ending this subsection, let us make an observation. For color ordered YM amplitudes, we have two bases: one is the $\mathrm{KK}$ basis with $(n-2)$ ! elements and another one is the BCJ basis with $(n-3)$ ! elements. As it has been seen in many examples, when we expand in the KK basis, the expansion coefficients can be properly chosen to be polynomial functions, while when we expand in the BCJ basis, the expansion coefficients will be rational functions in general. It is a simple but very useful observation. The reason for the polynomial functions rather than rational functions is clear: we must have some poles in these coefficients to match up with pole structures at both sides of the expansion.

\section{B. Expansion of SEYM amplitudes by YM amplitudes}

As explained in the Introduction, in this paper we will focus on the expansion of sEYM amplitudes in terms of YM amplitudes. As mentioned before, the expansion in the
KK basis has been conjectured first by gauge symmetry consideration[19] and then proved using the CHY integrands in [31]. In this subsection, we recall the recursive expansion of sEYM amplitudes $A_{|\mathrm{H}|}(1,2 \ldots r \| \mathrm{H})$ given in $[19,31,33]$

$$
\begin{aligned}
A_{r, \mathrm{H} \mid}^{\mathrm{EYM}} & (1,2 \ldots r \| \mathrm{H}) \\
= & \sum_{\boldsymbol{h} \mid \tilde{h}=\mathrm{H} \backslash h_{a}}\left[C_{h_{a}}(\boldsymbol{h}) A_{r+|\mathrm{H}|-|\tilde{h}|,|\tilde{h}|}^{\mathrm{EYM}}(1,\{2 \ldots r-1\}\right. \\
& \left.\left.\uplus\left\{\boldsymbol{h}, h_{a}\right\}, r \| \tilde{h}\right)\right],
\end{aligned}
$$

where the $H$ is the list of gravitons. The sum in (2.22) is over all partial ordered splitting of the list $\mathrm{H} \backslash h_{a}$, i.e., $\mathrm{H} \backslash h_{a}=\boldsymbol{h} \bigcup \tilde{\mathrm{h}}$, where different orderings of the subset $\boldsymbol{h}$ are treated to be different splittings while the orderings of $\tilde{h}$ do not matter. Suppose $\boldsymbol{h}=\left\{i_{s}, i_{s-1} \ldots i_{1}\right\}$, the coefficient $C_{h_{a}}(\boldsymbol{h})$ can be written as 


$$
C_{h_{a}}(\boldsymbol{h})=\epsilon_{h_{a}} \cdot f_{i_{1}} \cdot f_{i_{2}} \ldots f_{i_{s-1}} \cdot f_{i_{s}} \cdot Y_{i_{s}},
$$

where $\left(f_{i}\right)^{\mu \nu}=\left(k_{i}\right)^{\mu}\left(\epsilon_{i}\right)^{\nu}-\left(k_{i}\right)^{\nu}\left(\epsilon_{i}\right)^{\mu}$ is the field strength, and when $\boldsymbol{h}=\emptyset, C_{h_{a}}(\emptyset)=\epsilon_{h_{a}} \cdot Y_{h_{a}}$. The definition of $Y_{q}$ (2.4) is according to the set $\Xi=\{11,\{2 \ldots r-1\} \omega$ $\left.\left\{\boldsymbol{h}, h_{a}\right\}, r\right\}$ and the set $\beta=\left\{\boldsymbol{h}, h_{a}\right\}$.

The expression (2.22) is manifestly invariant under all permutations and gauge transformations of gravitons in the set $\mathrm{H} \backslash h_{a}$. The $h_{a}$ is special in the expansion and will be called the fiducial graviton. The full permutation and gauge invariances for all gravitons, although not explicitly indicated, are guaranteed by the generalized BCJ relations [38] (see explicit proof given in [42]). When we use (2.22) recursively, we can expand any sEYM amplitude in the KK basis of YM amplitudes with polynomial coefficients. In this paper, we will only use differential operators to reproduce the recursive expansion formula (2.22) and thus provide a new strategy to find the expansion.

Before ending this subsection, let us give an important remark. Because of the generalized BCJ relations (2.9), even imposing the polynomial conditions, the coefficients $C_{h_{a}}(\boldsymbol{h})$ 's in (2.23) cannot be unique, since one can always add these zero combinations. Such a freedom can easily be fixed by making some choices for expansion coefficients of some elements in the KK basis.

\section{Differential operators}

In [34], to study connections of amplitudes of different theories, some kinds of differential operators have been defined. The first kind is the trace operator $\mathcal{T}_{i j}$

$$
\mathcal{T}_{i j} \equiv \partial_{\epsilon_{i} \epsilon_{j}},
$$

where $\epsilon_{i} \epsilon_{j} \equiv \epsilon_{i} \cdot \epsilon_{j}$ is the Lorentz invariant contraction (similar understanding holds for all operators in this paper). The second kind is the insertion operator defined by

$$
\mathcal{T}_{i k j} \equiv \partial_{k_{i} \epsilon_{k}}-\partial_{k_{j} \epsilon_{k}} .
$$

As pointed out in [34], $\mathcal{T}_{i k j}$ itself is not a gauge invariant operator, but when it acts on those objects obtained after acting on a trace operator, it is effectively gauge invariant. Insertion operators will be extensively used in our paper. When it acts on the sEYM amplitudes, the operator $\mathcal{T}_{i k j}$ has the physical meaning of inserting the graviton $k$ between $i$ and $j$ when $i, j$ are nearby in a trace. If $i, j$ are not adjacent in a trace, for example, $\mathcal{T}_{i k(i+2)}$, we can write it as

$$
\mathcal{T}_{i k(i+2)}=\mathcal{T}_{i k(i+1)}+\mathcal{T}_{(i+1) k(i+2)} ;
$$

thus, the physical meaning is clear. If $i, j$ are not in the same trace, the physical meaning is not clear. The third kind of useful operator is the gauge invariant differential operator

$$
\mathcal{W}_{i} \equiv \sum_{v}\left(k_{i} v\right) \partial_{v \epsilon_{i}}
$$

where the sum is over all Lorentz contractions with $\epsilon_{i}$. When it is applied, $\epsilon_{i}$ is effectively replaced by $k_{i}$ in any expression. Thus, if an expression is gauge invariant, the action of $\mathcal{W}_{i}$ will give zero result. The fourth kind of useful operators are longitudinal operators defined via

$$
\mathcal{L}_{i} \equiv \sum_{j \neq i} k_{i} k_{j} \partial_{k_{j} \epsilon_{i}},
$$

and the related operators

$$
\mathcal{L}_{i j} \equiv-k_{i} k_{j} \partial_{\epsilon_{i} \epsilon_{j}}
$$

The roles of $\mathcal{L}_{i}$ and $\mathcal{L}_{i j}$ are to add derivative interactions in vertexes while getting rid of polarization vectors. Using them, we can define the operators $\mathcal{L}$ and $\tilde{\mathcal{L}}$ as [34]

$$
\mathcal{L} \equiv \prod_{i} \mathcal{L}_{i}, \quad \tilde{\mathcal{L}} \equiv \sum_{\rho \in \text { pair }} \prod_{i, j \in \rho} \mathcal{L}_{i j}
$$

At the algebraic level, the actions of $\mathcal{L}$ and $\tilde{\mathcal{L}}$ are different. However, if we consider the combination $\mathcal{L} \cdot \mathcal{T}_{a b} \mathbf{P f} \mathbf{f}^{\prime} \Psi$, and let subscripts of $\mathcal{L}_{i}$ 's and $\mathcal{L}_{i j}$ 's run through all legs in $\{1,2, \ldots, n\} \backslash\{a, b\}$, the effects of $\mathcal{L}$ and $\tilde{\mathcal{L}}$ are same and give a result which has a meaningful explanation. We will see it later.

As the simple applications of these kinds of differential operators, we derive three generalized relations for general EYM amplitudes. For simplicity we will use the sEYM amplitudes to demonstrate the idea. The first relation is the generalized color ordered reversed relation of EYM amplitudes:

$$
\begin{aligned}
& A^{\mathrm{EYM}}\left(1,2, \ldots,(n-1), n ;\left\{h_{g}\right\}\right) \\
& \quad=(-)^{n} A^{\mathrm{EYM}}\left(n,(n-1), \ldots, 2,1 ;\left\{h_{g}\right\}\right),
\end{aligned}
$$

which is reduced to the familiar color ordered reversed relation for pure YM amplitudes when $\left\{h_{g}\right\}=\emptyset$. To derive this, we use the fact that the ordering $(1,2, \ldots,(n-1), n)$ of sEYM amplitudes can be created by the following combination of operators:

$\mathcal{T}[1,2, \ldots,(n-1), n]=\mathcal{T}_{(n-2)(n-1) n} \cdots \mathcal{T}_{23 n} \mathcal{T}_{12 n} \mathcal{T}_{1 n}$,

acting on the amplitudes of gravity theory. Using the fact that $\mathcal{T}_{1 n}=\mathcal{T}_{n 1}$ and $\mathcal{T}_{i k j}=-\mathcal{T}_{j k i}$, we get 


$$
\begin{aligned}
& \mathcal{T}_{(n-2)(n-1) n} \cdots \mathcal{T}_{23 n} \mathcal{T}_{12 n} \mathcal{T}_{1 n} \\
& \quad=(-)^{n-2} \mathcal{T}_{n(n-1)(n-2)} \cdots \mathcal{T}_{n 32} \mathcal{T}_{n 21} \mathcal{T}_{n 1} \\
& \quad=(-)^{n-2} \mathcal{T}[n,(n-1), \ldots, 2,1] .
\end{aligned}
$$

Substituting it to (2.32), we find

$\mathcal{T}[1,2, \ldots,(n-1), n]=(-)^{n} \mathcal{T}[n,(n-1), \ldots, 2,1]$.

Then the relation (2.31) can be trivially obtained by applying (2.34) on the gravity amplitude $A^{G}(\{1, \ldots, n\} \cup$ $\left.\left\{h_{g}\right\}\right)$.

The second relation is the generalized photon decoupling relation of EYM amplitudes. By definition, we have

$$
\begin{aligned}
\mathcal{T}_{1 h n}= & \partial_{\epsilon_{h} \cdot k_{1}}-\partial_{\epsilon_{h} \cdot k_{n}} \\
= & \partial_{\epsilon_{h} \cdot k_{1}}-\partial_{\epsilon_{h} \cdot k_{2}}+\partial_{\epsilon_{h} \cdot k_{2}}-\partial_{\epsilon_{h} \cdot k_{3}}+\cdots \\
& +\partial_{\epsilon_{h} \cdot k_{n-1}}-\partial_{\epsilon_{h} \cdot k_{n}} \\
= & \mathcal{T}_{1 h 2}+\mathcal{T}_{2 h 3}+\cdots+\mathcal{T}_{(n-1) h n} .
\end{aligned}
$$

Putting them together gives

$$
\mathcal{T}_{1 h 2}+\mathcal{T}_{2 h 3}+\cdots+\mathcal{T}_{(n-1) h n}+\mathcal{T}_{n h 1}=0 .
$$

Applying this identity on $A^{\mathrm{EYM}}\left(1, \ldots, n ; h \cup\left\{h_{i}\right\}\right)$ leads to

$$
\sum_{ш} A^{\mathrm{EYM}}\left(1, h ш\{2, \ldots, n\} ;\left\{h_{i}\right\}\right)=0,
$$

which is the wanted generalized photon decoupling relation for EYM amplitudes.

The third relation is the generalized KK relation, which is given by

$$
\begin{aligned}
& A^{\mathrm{EYM}}\left(1, \alpha, n, \beta ;\left\{h_{g}\right\}\right) \\
& \quad=\sum_{\Psi}(-)^{n_{\beta}} A^{\mathrm{EYM}}\left(1, \alpha \omega \beta^{T}, n ;\left\{h_{g}\right\}\right) .
\end{aligned} .
$$

First we want to point out that when $\alpha$ is the empty subset, it reduces to the generalized color-ordered reserved relation (2.31). The proof of the relation (2.38) is extremely similar to the proof of (2.31). Suppose $\beta=\left\{b_{1}, b_{2}, \ldots, b_{r}\right\}$, we have

$$
\begin{aligned}
A^{\mathrm{EYM}} & \left(1, \alpha, n, \beta ;\left\{h_{g}\right\}\right) \\
& =A^{\mathrm{EYM}}\left(\alpha, n, \beta, 1 ;\left\{h_{g}\right\}\right) \\
& =\mathcal{T}_{n b_{1} 1} \mathcal{T}_{b_{1} b_{2} 1} \cdots \mathcal{T}_{b_{r-1} b_{r} 1} A^{\mathrm{EYM}}\left(\alpha, n, 1 ;\left\{h_{g}\right\} \cup \beta\right) \\
& =(-)^{n_{\beta}} \mathcal{T}_{1 b_{1} n} \mathcal{T}_{1 b_{2} b_{1}} \cdots \mathcal{T}_{1 b_{r} b_{r-1}} A^{\mathrm{EYM}}\left(1, \alpha, n ;\left\{h_{g}\right\} \cup \beta\right) .
\end{aligned}
$$

In the third step, the relation $\mathcal{T}_{i k j}=-\mathcal{T}_{j k i}$ and the cyclic symmetry have been used. To understand the meaning of the last line in (2.39), recall that all insertion operators are commutative and by the explanation of (2.26), the operator $\mathcal{T}_{1 b_{1} n}$ inserts $b_{1}$ at any position between 1 and $n$, and thus turns $A^{\mathrm{EYM}}\left(1, \alpha, n ;\left\{h_{g}\right\} \cup \beta\right)$ to $A^{\mathrm{EYM}}\left(1, \alpha ш b_{1}, n ;\left\{h_{g}\right\} \cup\right.$ $\left.\beta \backslash \beta_{1}\right)$. The next action of $\mathcal{T}_{1 b_{2} b_{1}}$ gives $A^{\mathrm{EYM}}(1$, $\left.\alpha ш\left\{\beta_{2}, b_{1}\right\}, n ;\left\{h_{g}\right\} \cup \beta \backslash\left\{b_{1}, \beta_{2}\right\}\right)$. Continuing the action until the last one, the last line in (2.39) gives $(-)^{n_{\beta}} A^{\mathrm{EYM}}\left(1, \alpha ш \beta^{T}, n ;\left\{h_{g}\right\}\right)$; thus, the generalized $\mathrm{KK}$ relation (2.38) is obtained.

\section{EXPANSION IN THE KK BASIS BY DIFFERENTIAL OPERATORS}

In this section, we will propose a new strategy to derive the expansion of sEYM amplitudes in the KK basis of YM amplitudes by differential operators given in [34]. Before doing so, we make some remarks:

(1) Although it is well known that SEYM amplitudes can be expanded in terms of YM amplitudes, we do not need to assume this in the beginning. From the presentation in this section, we will see that assuming the factorization of polarization tensors $\epsilon_{i}^{\mu \nu}$ of gravitons into two polarization vectors $\epsilon$ and $\tilde{\epsilon}$ of gluons (i.e., $\epsilon_{i}^{\mu \nu}=\epsilon_{i}^{\mu} \tilde{\epsilon}_{i}^{\nu}$ ), the web connections given in [34] will naturally lead to the expansion of sEYM amplitudes in terms of YM amplitudes.

(2) Although, in general, the expansion coefficients in the KK basis can be rational functions as in $[25,26]$, one can arrange them properly using the generalized $\mathrm{BCJ}$ relations (2.9) to get polynomial coefficients as written in (2.22). If we impose the condition that the expansion coefficients are polynomial, the KK basis can be treated as independent up to the generalized BCJ relations (2.9). ${ }^{5}$ Under this understanding, we see that the expansion coefficients in the KK basis have the freedom caused by the generalized BCJ relations.

(3) Since we have assumed the factorization of polarization tensors of gravitons, we consider building blocks for the polarization vectors $\epsilon$. For a given graviton, the expansion coefficients must be the function of Lorentz invariant combinations $\left(k_{i} \cdot k_{j}\right)$, $\left(\epsilon_{i} \cdot k_{j}\right)$, as well as $\left(\epsilon_{i} \cdot \epsilon_{j}\right) .^{6}$ Among them, $\left(\epsilon_{i} \cdot k_{j}\right)$ and $\left(\epsilon_{i} \cdot \epsilon_{j}\right)$ are the building blocks for the expansion when applying the differential operators. One reason for using these building blocks is that we can

\footnotetext{
${ }^{5}$ If we use the language of algebraic geometry, generalized BCJ relations (2.9) will generate an ideal in the polynomial ring of $(n-2)$ ! variables. The KK basis will be the independent basis in the quotient ring.

${ }^{6}$ Here we have excluded the contraction $\epsilon_{i} \cdot \tilde{\epsilon}_{i}$. In other words, we have assumed that two polarization vectors coming from the same graviton should not contract with each other. This is a very natural assumption and could be seen from the traditional Feynman rules of gravitons.
} 
transform the differential equations of expansion to linear algebraic equations of unknown coefficients of building blocks. One will see that such a choice of building blocks will naturally lead to the expansion in the KK basis.

(4) The mass dimension of expansion coefficients is $|H|$, the number of gravitons.

(5) For the expansion of sEYM amplitudes $A_{n, m}^{\mathrm{EYM}}(1, \ldots$, $\left.n ;\left\{h_{1}, \ldots, h_{m}\right\}\right)$, the KK basis is not general since the ordering of $\{1,2, \ldots, n\}$ will be kept for each amplitude of the basis with nonzero coefficient. This fact can easily be understood from the pole structures of both sides and be proved using the insertion operators. Keeping only these KK bases with the ordering $\{1,2, \ldots, n\}$ has also partially fixed the freedoms caused by the generalized BCJ relations (2.9).

The above five points come from general considerations. Based on them, the structure of this section is the following. In the first subsection, we consider the SEYM amplitudes with only one graviton. The calculations in this part are straightforward. In the second subsection, we consider the sEYM amplitudes with two gravitons. This part is very important because it contains the recursive strategy used in the third subsection for general situations. Based on the idea in the second part, the recursive expansion for sEYM amplitudes with an arbitrary number of gravitons has been presented in the third subsection. Thus, we have shown that using differential operators, we could indeed derive the expansion of sEYM amplitudes in the KK basis of YM amplitudes as given in (2.22).

\section{A. The case with one graviton}

According to the principles of Lorentz invariance and momentum conservation, the sEYM amplitude with one graviton $A_{n, 1}^{\mathrm{EYM}}\left(1, \ldots, n ; h_{1}\right)$ can always be written as

$$
A_{n, 1}^{\mathrm{EYM}}\left(1, \ldots, n ; h_{1}\right)=\sum_{i=1}^{n-1}\left(\epsilon_{h_{1}} \cdot k_{i}\right) \tilde{B}_{i},
$$

where $\left(\epsilon_{h_{1}} \cdot k_{i}\right)$ 's ${ }^{7}$ constitute linearly independent building blocks with coefficients $\tilde{B}_{i}$ being functions of $\tilde{\epsilon}_{h_{1}}$ and $\left(k_{i} \cdot k_{j}\right)$. By our experiences, to simplify the calculation we can choose a new basis of building blocks and the expansion is

$$
A_{n, 1}^{\mathrm{EYM}}\left(1, \ldots, n ; h_{1}\right)=\sum_{i=1}^{n-1}\left(\epsilon_{h_{1}} \cdot K_{i}\right) B_{i},
$$

with $K_{i}=\sum_{j=1}^{i} k_{j}, i=1, \ldots, n-1$.

\footnotetext{
${ }^{7}$ The $\epsilon_{h_{1}} \cdot k_{n}$ is not independent by the momentum conservation.
}

Now we use differential operators to determine $B_{i}$ 's. To do so, we apply insertion operators $\mathcal{T}_{a h_{1}(a+1)}$ with $a=$ $1, \ldots, n-1$ to the expansion (3.2). Since $\mathcal{T}_{a h_{1}(a+1)}=$ $\partial_{\epsilon_{h_{1}} \cdot k_{a}}-\partial_{\epsilon_{h_{1}} \cdot k_{a+1}}$ does not act on $B_{i}$, we have

$$
\begin{aligned}
& \mathcal{T}_{a h_{1}(a+1)} A_{n, 1}^{\mathrm{EYM}}\left(1, \ldots, n ; h_{1}\right) \\
& =A_{n+1}^{\mathrm{YM}}\left(1, \ldots, a, h_{1}, a+1, \ldots, n-1\right) \\
& =\sum_{i=1}^{n-1}\left\{\mathcal{T}_{a h_{1}(a+1)}\left(\epsilon_{h_{1}} \cdot K_{i}\right)\right\} B_{i} \\
& =\sum_{i=1}^{n-1} \delta_{a, i} B_{i}=B_{a} .
\end{aligned}
$$

The above manipulations show that after applying differential operators to the building blocks, we obtain linear algebraic equations for these $B_{i}$ 's. With the smart choice of building blocks, these linear equations are very easy to solve

$$
B_{i}=A_{n+1}^{\mathrm{YM}}\left(1, \ldots, i, h_{1}, i+1, \ldots, n-1\right)
$$

Here is a subtlety for the solution (3.4). As we have remarked, the KK basis is independent only up to the generalized BCJ relations (2.9). Thus, in principle, one can add these terms at the right-hand side of (3.4). But with the polynomial condition as well as the mass dimension condition of coefficients, one can see that (3.4) is the only allowed solution. Similar phenomena will appear in later subsections and we will not discuss them further. Putting the solutions back, we get

$$
\begin{aligned}
& A_{n, 1}^{\mathrm{EYM}}\left(1, \ldots, n ; h_{1}\right) \\
& \quad=\sum_{i=1}^{n-1}\left(\epsilon_{h_{1}} \cdot K_{i}\right) A_{n+1}^{\mathrm{YM}}\left(1, \ldots, i, h_{1}, i+1, \ldots, n-1\right) \\
& \quad=\sum_{\varpi}\left(\epsilon_{h_{1}} \cdot Y_{h_{1}}\right) A_{n+1}^{\mathrm{YM}}\left(1,\{2, \ldots, n-1\} ш\left\{h_{1}\right\}, n\right),
\end{aligned}
$$

where in the second line we have used the definition of $w$ and $Y_{h_{1}}$ [see (2.4)].

Although this example is trivial, it reveals the essence of our strategy: (1) The expansion in the KK basis of YM amplitudes is the natural consequence of web connections established in [34] without relying other information such as the double copy form (1.3). (2) The differential operators act on building blocks only, and we get linear algebraic equations for unknown coefficients, which are much easier to solve than differential equations. This transmutation reflects the important role of building blocks. 


\section{B. The case with two gravitons}

Now we move to the less trivial case $A_{n, 2}^{\mathrm{EYM}}$. This example will show how to generalize our strategy to arbitrary amplitudes $A_{n, m}^{\mathrm{EYM}}$. As in the previous subsection, we expand $A_{n, 2}^{\mathrm{EYM}}$ according to the building blocks of $\epsilon_{h_{1}}$ as

$$
A_{n, 2}^{\mathrm{EYM}}\left(1, \ldots, n ; h_{1}, h_{2}\right)=\sum_{i=1}^{n-1}\left(\epsilon_{h_{1}} \cdot K_{i}\right) B_{i}+\left(\epsilon_{h_{1}} \cdot k_{h_{2}}\right)\left(\epsilon_{h_{2}} \cdot D_{h_{2}}\right)+\left(\epsilon_{h_{1}} \cdot \epsilon_{h_{2}}\right) E_{h_{2}} \cdot
$$

To determine $B_{i}$, we use insertion operators $\mathcal{T}_{a h_{1}(a+1)}$ with $a=1, \ldots, n-1$. After acting on both sides, we arrive

$$
\mathcal{T}_{a h_{1}(a+1)} A_{n, 2}^{\mathrm{EYM}}\left(1, \ldots, n ; h_{1}, h_{2}\right)=A_{n+1,1}^{\mathrm{EYM}}\left(1, \ldots, a, h_{1}, a+1, \ldots, n ; h_{2}\right)=B_{a} .
$$

Putting the solutions back, (3.6) becomes

$A_{n, 2}^{\mathrm{EYM}}\left(1, \ldots, n ; h_{1}, h_{2}\right)=\sum_{\Psi}\left(\epsilon_{h_{1}} \cdot Y_{h_{1}}\right) A_{n+1,1}^{\mathrm{EYM}}\left(1,\{2, \ldots, n-1\} ш\left\{h_{1}\right\}, n ; h_{2}\right)+\left(\epsilon_{h_{1}} \cdot k_{h_{2}}\right)\left(\epsilon_{h_{2}} \cdot D_{h_{2}}\right)+\left(\epsilon_{h_{1}} \cdot \epsilon_{h_{2}}\right) E_{h_{2}}$.

To determine the remaining two variables $D_{h_{2}}, E_{h_{2}}$, we use the gauge invariance of the graviton $h_{2}{ }^{8}$ There are two approaches. The first approach is that since the first term at the rhs of (3.8) is $h_{2}$ gauge invariant already, after $\epsilon_{h_{2}} \rightarrow k_{h_{2}}$ in the remaining two terms, we get

$$
\left(\epsilon_{h_{1}} \cdot k_{h_{2}}\right)\left(k_{h_{2}} \cdot D_{h_{2}}\right)+\left(\epsilon_{h_{1}} \cdot k_{h_{2}}\right) E_{h_{2}}=0 \Rightarrow\left(k_{h_{2}} \cdot D_{h_{2}}\right)+E_{h_{2}}=0
$$

The second approach is to consider the commutation relation of insertion operators and gauge invariant operators [see (2.25) and (2.27) $]^{9}$

$$
\left[\mathcal{T}_{i j k}, \mathcal{W}_{l}\right]=\delta_{i l} \mathcal{T}_{i j}-\delta_{k l} \mathcal{T}_{j k}
$$

where $\mathcal{T}_{j k}$ is the trace operator (2.24). For the current case, we apply the commutation relation $\left[\mathcal{T}_{h_{2} h_{1} n}, \mathcal{W}_{h_{2}}\right]=\mathcal{T}_{h_{2} h_{1}}$ to both sides of (3.8) and get

$$
\begin{aligned}
{\left[\mathcal{T}_{h_{2} h_{1} n}, \mathcal{W}_{h_{2}}\right] A_{n, 2}^{\mathrm{EYM}}\left(1, \ldots, n ; h_{1}, h_{2}\right) } & =-\mathcal{W}_{h_{2}} \mathcal{T}_{h_{2} h_{1} n} A_{n, 2}^{\mathrm{EYM}}\left(1, \ldots, n ; h_{1}, h_{2}\right)=-\left(k_{h_{2}} \cdot D_{h_{2}}\right) \\
\mathcal{T}_{h_{2} h_{1}} A_{n, 2}^{\mathrm{EYM}}\left(1, \ldots, n ; h_{1}, h_{2}\right) & =E_{h_{2}} .
\end{aligned}
$$

No matter which approach is used, now (3.8) becomes

$$
A_{n, 2}^{\mathrm{EYM}}\left(1, \ldots, n ; h_{1}, h_{2}\right)=\sum_{\Psi}\left(\epsilon_{h_{1}} \cdot Y_{h_{1}}\right) A_{n+1,1}^{\mathrm{EYM}}\left(1,\{2, \ldots, n-1\} ш\left\{h_{1}\right\}, n ; h_{2}\right)+\left(\epsilon_{h_{1}} \cdot f_{h_{2}} \cdot D_{h_{2}}\right),
$$

where the gauge invariance of $h_{2}$ is manifest. To determine the last variable $D_{h_{2}}$, we expand it according to the building blocks of $\epsilon_{h_{2}}$ as

$A_{n, 2}^{\mathrm{EYM}}\left(1, \ldots, n ; h_{1}, h_{2}\right)=\sum_{\Psi}\left(\epsilon_{h_{1}} \cdot Y_{h_{1}}\right) A_{n+1,1}^{\mathrm{EYM}}\left(1,\{2, \ldots, n-1\} ш\left\{h_{1}\right\}, n ; h_{2}\right)+\sum_{i=1}^{n-1}\left(\epsilon_{h_{1}} \cdot f_{h_{2}} \cdot K_{i}\right) H_{i}+\left(\epsilon_{h_{1}} \cdot f_{h_{2}} \cdot k_{h_{1}}\right) H_{h_{1}} \cdot$

To determine the $H_{i}$ 's, we apply the combinations $\mathcal{T}_{a h_{2}(a+1)} \mathcal{I}_{h_{2} h_{1}(a+1)}, a=1, \ldots, n-1$ of two insertion operators, and get

\footnotetext{
${ }^{8}$ The gauge invariance of graviton $h_{i}$ means that the Ward's identity, i.e., the amplitude, vanishes under the replacement $\epsilon_{h_{i}} \rightarrow k_{h_{i}}$.

${ }^{9}$ Although for this simple example, the first approach is simpler, but when trying to generalize to general cases, the second approach is more suitable.
} 


$$
\begin{aligned}
& \mathcal{T}_{a h_{2}(a+1)} \mathcal{T}_{h_{2} h_{1}(a+1)} A_{n, 2}^{\mathrm{EYM}}\left(1, \ldots, n ; h_{1}, h_{2}\right)=A_{n+2}^{\mathrm{YM}}\left(1, \ldots, a, h_{2}, h_{1}, a+1, n\right) \\
& =\mathcal{T}_{a h_{2}(a+1)}\left\{-\sum_{i=a+1}^{n-1} A_{n+1,1}^{\mathrm{EYM}}\left(1, \ldots, i, h_{1}, i+1, \ldots, n ; h_{2}\right)+\sum_{i=1}^{n-1}\left(\epsilon_{h_{2}} \cdot K_{i}\right) H_{i}+\left(\epsilon_{h_{2}} \cdot k_{h_{1}}\right) H_{h_{1}}\right\} \\
& =-\sum_{i=a+1}^{n-1} A_{n+2}^{\mathrm{YM}}\left(1, \ldots, a, h_{2}, a+1, \ldots, i, h_{1}, i+1, \ldots, n\right)+H_{a} .
\end{aligned}
$$

From it we can solve

$$
H_{a}=\sum_{\Psi} A_{n+2}^{\mathrm{YM}}\left(1, \ldots, a, h_{2},\{a+1, \ldots, n-1\} ш\left\{h_{1}\right\}, n\right),
$$

so the expansion becomes

$$
\begin{aligned}
A_{n, 2}^{\mathrm{EYM}}\left(1, \ldots, n ; h_{1}, h_{2}\right)= & \sum_{\varpi}\left(\epsilon_{h_{1}} \cdot Y_{h_{1}}\right) A_{n+1,1}^{\mathrm{EYM}}\left(1,\{2, \ldots, n-1\} ш\left\{h_{1}\right\}, n ; h_{2}\right)+\left(\epsilon_{h_{1}} \cdot f_{h_{2}} \cdot k_{h_{1}}\right) H_{h_{1}} \\
& +\sum_{\Psi}\left(\epsilon_{h_{1}} \cdot f_{h_{2}} \cdot Y_{h_{2}}\right) A_{n+2}^{\mathrm{YM}}\left(1,\{2, \ldots, n-1\} ш\left\{h_{2}, h_{1}\right\}, n\right) .
\end{aligned}
$$

Now only the $H_{h_{1}}$ is unknown. This one is manifestly gauge invariant and symmetric because $\left(\epsilon_{h_{1}} \cdot f_{h_{2}} \cdot k_{h_{1}}\right) H_{h_{1}}=$ $\frac{1}{2} \operatorname{tr}\left(f_{h_{1}} f_{h_{2}}\right) H_{h_{1}}$. To fix it, we use the special operator $\mathcal{T}_{j h_{1} h_{2}} \mathcal{T}_{j h_{2} h_{1}}$. While acting on the right-hand side of (3.16) it gives

$$
\begin{aligned}
R= & \mathcal{T}_{j h_{1} h_{2}} \mathcal{T}_{j h_{2} h_{1}}\left\{\sum_{\Psi}\left(\epsilon_{h_{1}} \cdot Y_{h_{1}}\right) A_{n+1,1}^{\mathrm{EYM}}\left(1,\{2, \ldots, n-1\} w\left\{h_{1}\right\}, n ; h_{2}\right)\right. \\
& \left.+\sum_{\Psi}\left(\epsilon_{h_{1}} \cdot f_{h_{2}} \cdot Y_{h_{2}}\right) A_{n+2}^{\mathrm{YM}}\left(1,\{2, \ldots, n-1\} ш\left\{h_{2}, h_{1}\right\}, n\right)+\left(\epsilon_{h_{1}} \cdot f_{h_{2}} \cdot k_{h_{1}}\right) H_{h_{1}}\right\}=H_{h_{1}},
\end{aligned}
$$

when acting on the left-hand side, the physical meaning of "insertion" is not clear at the level of amplitudes. However, as shown in $[35,36]$, its action at the level of CHY integrands is still clear. Doing it leads to

$$
L=\mathcal{T}_{j h_{1} h_{2}} \mathcal{T}_{j h_{2} h_{1}} A_{n, 2}^{\mathrm{EYM}}\left(1, \ldots, n ; h_{1}, h_{2}\right)=\int d \mu \mathrm{PT}(1,2, \ldots, n)\left\{\mathcal{T}_{j h_{1} h_{2}} \mathcal{T}_{j h_{2} h_{1}} \mathrm{Pf}_{H}\right\} \operatorname{Pf}^{\prime} \Psi
$$

To continue, we use the Pfaffian expansion given in $[43,44]$ and keep only terms contributing under these operators. The related terms ${ }^{10}$ are $\left(\Psi_{\left(h_{1}\right)} \Psi_{\left(h_{2}\right)}-\Psi_{\left(h_{1} h_{2}\right)}\right)$ with

$$
\Psi_{\left(h_{1}\right)}=C_{h_{1} h_{1}}=-\sum_{a \neq h_{1}} \frac{\epsilon_{h_{1}} \cdot k_{a}}{\sigma_{h_{1} a}}, \quad \Psi_{\left(h_{2}\right)}=C_{h_{2} h_{2}}=-\sum_{a \neq h_{2}} \frac{\epsilon_{h_{2}} \cdot k_{a}}{\sigma_{h_{2} a}}, \quad \Psi_{\left(h_{1} h_{2}\right)}=\frac{\operatorname{tr}\left(f_{h_{1}} \cdot f_{h_{2}}\right)}{2 \sigma_{h_{1} h_{2}} \sigma_{h_{2} h_{1}}} .
$$

Using them, we find

\footnotetext{
${ }^{10}$ For more details, please see Appendix A.
} 


$$
\begin{aligned}
& \mathcal{T}_{j h_{1} h_{2}} \mathcal{T}_{j h_{2} h_{1}} \operatorname{Pf}\left(\Psi_{H}\right) \\
& =\mathcal{T}_{j h_{1} h_{2}} \mathcal{T}_{j h_{2} h_{1}}\left\{\sum_{a \neq h_{1}} \frac{\epsilon_{h_{1}} \cdot k_{a}}{\sigma_{h_{1} a}} \sum_{b \neq h_{2}} \frac{\epsilon_{h_{2}} \cdot k_{b}}{\sigma_{h_{2} b}}-\frac{\operatorname{tr}\left(f_{h_{1}} \cdot f_{h_{2}}\right)}{2 \sigma_{h_{1} h_{2}} \sigma_{h_{2} h_{1}}}\right\} \\
& =\left(\frac{1}{\sigma_{h_{1} j}}-\frac{1}{\sigma_{h_{1} h_{2}}}\right)\left(\frac{1}{\sigma_{h_{2} j}}-\frac{1}{\sigma_{h_{2} h_{1}}}\right)-\frac{1}{\sigma_{h_{1} h_{2}} \sigma_{h_{2} h_{1}}} \\
& =\frac{\sigma_{j h_{2}}}{\sigma_{h_{1} j} \sigma_{h_{1} h_{2}}} \frac{\sigma_{j h_{1}}}{\sigma_{h_{2} j} \sigma_{h_{2} h_{1}}}-\frac{1}{\sigma_{h_{1} h_{2}} \sigma_{h_{2} h_{1}}}=0 .
\end{aligned}
$$

Since the left-hand side is zero, we find $H_{h_{1}}=0$.

Assembling all pieces together, we obtain the expansion of $A_{n, 2}^{\mathrm{EYM}}$ as

$$
\begin{aligned}
A_{n, 2}^{\mathrm{EYM}}\left(1, \ldots, n ; h_{1}, h_{2}\right) \\
=\sum_{\amalg}\left(\epsilon_{h_{1}} \cdot Y_{h_{1}}\right) A_{n+1,1}^{\mathrm{EYM}}\left(1,\{2, \ldots, n-1\} ш\left\{h_{1}\right\}, n ; h_{2}\right) \\
\quad+\sum_{\Psi}\left(\epsilon_{h_{1}} \cdot f_{h_{2}} \cdot Y_{h_{2}}\right) A_{n+2}^{\mathrm{YM}}\left(1,\{2, \ldots, n-1\} ш\left\{h_{2}, h_{1}\right\}, n\right) .
\end{aligned}
$$

It is a recursive expansion of $A_{n, 2}^{\mathrm{EYM}}$ by $A_{n+1,1}^{\mathrm{EYM}}$ and $A_{n+2}^{\mathrm{YM}}$. We can get the complete expansion of $A_{n, 2}^{\mathrm{EYM}}$ in terms of purely YM amplitudes by putting back the known expansion of $A_{n+1,1}^{\mathrm{EYM}}$ in (3.5).

From the above calculations we see again that the expansion of sEYM amplitudes to the KK basis of YM amplitudes is the natural consequence of web connections established in [34] by differential operators. Furthermore, we see that to find the expansion, we could do it step by step. At the first step, we organize the expansion according to the building blocks of $\epsilon_{h_{1}}$ and then by using insertion and gauge invariant differential operators to determine some coefficients. At the second step, we organize the remaining unknown variables according to the building blocks of the polarization vector of next gravitons, and then solve some variables again. Repeating the procedure, we will finally determine the expansion. In the next subsection, we will see more details about the iterative operations.

A nontrivial thing in the above derivation is the possible appearance of the term $\left(\epsilon_{h_{1}} \cdot f_{h_{2}} \cdot k_{h_{1}}\right) H_{h_{1}}=$ $\frac{1}{2} \operatorname{tr}\left(f_{h_{1}} f_{h_{2}}\right) H_{h_{1}}$. It satisfies all general conditions, such as the multilinearity of $\epsilon_{h_{i}}$, gauge invariance, polynomial, etc. To show its vanishing, we need to consider the action of differential operators at the level of $\mathrm{CHY}$ integrands instead of at the level of amplitudes directly. The vanishing result has been carefully proved in Appendix A, which may imply some interesting things at the level of sEYM amplitudes.

\section{The case with $\boldsymbol{m}$ gravitons}

Having outlined our derivation strategy in the previous subsection, we consider the expansion of arbitrary sEYM amplitudes $A_{n, m}^{\mathrm{EYM}}$. Let us begin with the expansion according to the building blocks of graviton $h_{1}$

$$
A_{n, m}\left(1,2, \ldots, n ;\left\{h_{1}, \ldots, h_{m}\right\}\right)=\sum_{i=1}^{n-1}\left(\epsilon_{h_{1}} \cdot K_{i}\right) B_{i}+\sum_{j=2}^{m}\left(\epsilon_{h_{1}} \cdot k_{h_{j}}\right)\left(\epsilon_{h_{j}} \cdot D_{h_{j}}\right)+\sum_{j=2}^{m}\left(\epsilon_{h_{1}} \cdot \epsilon_{h_{j}}\right) E_{h_{j}}
$$

At the first step we apply the insertion operators $\mathcal{T}_{a h_{1}(a+1)}$ with $a=1, \ldots, n-1$ to (3.22) and get

$$
\mathcal{T}_{a h_{1}(a+1)} A_{n, m}(1,2, \ldots, n ; \mathbf{h})=A_{n+1, m-1}\left(1,2, \ldots, a, h_{1}, a+1, \ldots, n ; \mathbf{h} /\left\{h_{1}\right\}\right)=B_{a},
$$

where we use $\mathbf{h}$ to denote the set of gravitons $\left\{h_{1}, \ldots, h_{m}\right\}$. Thus, (3.22) becomes

$$
\begin{aligned}
A_{n, m}(1,2, \ldots, n ; \mathbf{h})= & \sum_{\Psi}\left(\epsilon_{h_{1}} \cdot Y_{h_{1}}\right) A_{n+1, m-1}\left(1,\{2, \ldots, n-1\} \omega\left\{h_{1}\right\}, n ; \mathbf{h} /\left\{h_{1}\right\}\right) \\
& +\sum_{j=2}^{m}\left(\epsilon_{h_{1}} \cdot k_{h_{j}}\right)\left(\epsilon_{h_{j}} \cdot D_{h_{j}}\right)+\sum_{j=2}^{m}\left(\epsilon_{h_{1}} \cdot \epsilon_{h_{j}}\right) E_{h_{j}}
\end{aligned}
$$

At the second step, using the gauge invariance conditions, i.e., the commutation relations $\left[\mathcal{T}_{h_{a} h_{1} h}, \mathcal{W}_{h_{a}}\right]=\mathcal{T}_{h_{a} h_{1}}$ with $a=2, \ldots, m$ to (3.24), we get

$$
\left[\mathcal{T}_{h_{a} h_{1} h}, \mathcal{W}_{h_{a}}\right] A_{n, m}(1,2, \ldots, n ; \mathbf{h})=-\left(k_{h_{a}} \cdot D_{h_{a}}\right)=\mathcal{T}_{h_{a} h_{1}} A_{n, m}(1,2, \ldots, n ; \mathbf{h})=E_{h_{a}},
$$

thus (3.24) becomes 


$$
A_{n, m}(1,2, \ldots, n ; \mathbf{h})=\sum_{\Psi}\left(\epsilon_{h_{1}} \cdot Y_{h_{1}}\right) A_{n+1, m-1}\left(1,\{2, \ldots, n-1\} w\left\{h_{1}\right\}, n ; \mathbf{h} /\left\{h_{1}\right\}\right)+\sum_{j=2}^{m}\left(\epsilon_{h_{1}} \cdot f_{h_{j}} \cdot D_{h_{j}}\right) .
$$

One needs to notice that $D_{h_{j}}$ 's are required to be gauge invariant for gravitons $h \neq h_{1}, h_{j}{ }^{11}$

To determine the $D_{h_{j}}$, we need to expand $\left(\epsilon_{h_{j}} \cdot D_{h_{j}}\right)$ in (3.24) further according to $h_{j}$ 's building blocks

$$
\left(\epsilon_{h_{j}} \cdot D_{h_{j}}\right)=\sum_{j_{2}=2, j_{2} \neq j}^{m}\left(\epsilon_{h_{j}} \cdot k_{h_{j_{2}}}\right)\left(\epsilon_{h_{j_{2}}} \cdot D_{h_{j} h_{j_{2}}}\right)+\sum_{j_{2}=2, j_{2} \neq j}^{m}\left(\epsilon_{h_{j}} \cdot \epsilon_{h_{j_{2}}}\right) E_{h_{j} h_{j_{2}}}+\sum_{i=1}^{n-1}\left(\epsilon_{h_{j}} \cdot K_{i}\right) B_{h_{j} i}+\left(\epsilon_{h_{j}} \cdot k_{h_{1}}\right) B_{h_{j} h_{1}} .
$$

Because of the gauge invariance of $D_{h_{j}}$, we can apply the commutation relations $\left[\mathcal{T}_{h_{a} h_{j} n}, \mathcal{W}_{h_{a}}\right]=\mathcal{T}_{h_{a} h_{j}}($ with $a=2, \ldots, m$, but $a \neq j)$ to (3.27) and get $E_{h_{j} h_{j_{2}}}=-\left(k_{h_{j_{2}}} \cdot D_{h_{j} h_{j_{2}}}\right)$. Then (3.27) becomes

$$
\left(\epsilon_{h_{j}} \cdot D_{h_{j}}\right)=\sum_{j_{2}=2, j_{2} \neq j}^{m}\left(\epsilon_{h_{j}} \cdot f_{h_{j_{2}}} \cdot D_{h_{j} h_{j_{2}}}\right)+\sum_{i=1}^{n-1}\left(\epsilon_{h_{j}} \cdot K_{i}\right) B_{h_{j} i}+\left(\epsilon_{h_{j}} \cdot k_{h_{1}}\right) B_{h_{j} h_{1}},
$$

and (3.26) becomes

$$
\begin{aligned}
A_{n, m}(1,2, \ldots, n ; \mathbf{h})= & \sum_{\Psi}\left(\epsilon_{h_{1}} \cdot Y_{h_{1}}\right) A_{n+1, m-1}\left(1,\{2, \ldots, n-1\} ш\left\{h_{1}\right\}, n ; \mathbf{h} /\left\{h_{1}\right\}\right)+\sum_{j=2}^{m} \sum_{j_{2}=2, j_{2} \neq j}^{m}\left(\epsilon_{h_{1}} \cdot f_{h_{j}} \cdot f_{h_{j_{2}}} \cdot D_{h_{j} h_{j_{2}}}\right) \\
& +\sum_{j=2}^{m} \sum_{i=1}^{n-1}\left(\epsilon_{h_{1}} \cdot f_{h_{j}} \cdot K_{i}\right) B_{h_{j} i}+\sum_{j=2}^{m}\left(\epsilon_{h_{1}} \cdot f_{h_{j}} \cdot k_{h_{1}}\right) B_{h_{j} h_{1}} .
\end{aligned}
$$

To determine $B_{h_{j} i}$ 's we apply the products of two insertion operators, i.e., $\mathcal{T}_{a h_{j}(a+1)} \mathcal{I}_{h_{j} h_{1}(a+1)}$ with $a=1, \ldots, n-1$ to $(3.29)$

$$
\begin{aligned}
\mathcal{T}_{a h_{j}(a+1)} \mathcal{T}_{h_{j} h_{1}(a+1)} A_{n, m}(1,2, \ldots, n ; \mathbf{h}) & =A_{n+2, m-2}\left(1,2, \ldots, a, h_{j}, h_{1}, a+1, \ldots, n ; \mathbf{h} /\left\{h_{1}, h_{j}\right\}\right) \\
& =-\sum_{i=a+1}^{m} A_{n+2, m-2}\left(1,2, \ldots, a, h_{j}, a+1, i, h_{1}, i+1, \ldots, n ; \mathbf{h} /\left\{h_{1}, h_{j}\right\}\right)+B_{h_{j} a},
\end{aligned}
$$

thus obtaining $B_{h_{j} a}=A_{n+2, m-2}\left(1,2, \ldots, a, h_{j},\{a+1, \ldots, n-1\} w\left\{h_{1}\right\}, n ; \mathbf{h} /\left\{h_{1}, h_{j}\right\}\right)$. Next, doing a similar thing (i.e., by acting on proper operators $\mathcal{T}_{k h_{1} h_{j}} \mathcal{T}_{k h_{j} h_{1}}$ on the CHY integrand) like in the previous subsection, one can show the vanishing of $B_{h_{j} h_{1}}$. Thus, (3.29) becomes

$$
\begin{aligned}
A_{n, m}(1,2, \ldots, n ; \mathbf{h})= & \sum_{\varpi}\left(\epsilon_{h_{1}} \cdot Y_{h_{1}}\right) A_{n+1, m-1}\left(1,\{2, \ldots, n-1\} ш\left\{h_{1}\right\}, n ; \mathbf{h} /\left\{h_{1}\right\}\right) \\
& +\sum_{j=2}^{m} \sum_{\varpi}\left(\epsilon_{h_{1}} \cdot f_{h_{j}} \cdot Y_{h_{j}}\right) A_{n+2, m-2}\left(1,\{2, \ldots, n-1\} w\left\{h_{j}, h_{1}\right\}, n ; \mathbf{h} /\left\{h_{1}, h_{j}\right\}\right) \\
& +\sum_{j=2}^{m} \sum_{j_{2}=2, j_{2} \neq j}^{m}\left(\epsilon_{h_{1}} \cdot f_{h_{j}} \cdot f_{h_{j_{2}}} \cdot D_{h_{j} h_{j_{2}}}\right),
\end{aligned}
$$

where $D_{h_{j} h_{j_{2}}}$ 's are also gauge invariant for remaining gravitons.

\footnotetext{
${ }^{11}$ It is because $A_{n+1, m-1}\left(1,\{2, \ldots, n-1\} ш\left\{h_{1}\right\}, n ; \mathbf{h} /\left\{h_{1}\right\}\right)$ is gauge invariant for the remaining gravitons and $\left(\epsilon_{h_{1}} \cdot f_{h_{j}} \cdot D_{h_{j}}\right)$ 's are linearly independent.
} 
Now we compare (3.26) and (3.31). We see that to get (3.31) from (3.26), we need to just do the replacement

$$
D_{h_{j}}^{\mu} \rightarrow \sum_{\Psi} Y_{h_{j}}^{\mu} A_{n+2, m-2}\left(1,\{2, \ldots, n-1\} \Psi\left\{h_{j}, h_{1}\right\}, n ; \mathbf{h} /\left\{h_{1}, h_{j}\right\}\right)+\sum_{j_{2}=2, j_{2} \neq j}^{m}\left(f_{h_{j_{2}}} \cdot D_{h_{j} h_{j_{2}}}\right)^{\mu} .
$$

This replacement is derived by using the proper products of insertion operators, which act either at the amplitude level or at the CHY-integrand level as shown in above. Now the pattern is clear. To expand $\left(\epsilon_{h_{j_{2}}} \cdot D_{h_{j} h_{j_{2}}}\right)$, we act on with the products of three insertion operators and find the result is equivalent to the replacement

$$
D_{h_{j} h_{j_{2}}}^{\mu} \rightarrow \sum_{\Psi} Y_{h_{j_{2}}}^{\mu} A_{n+3, m-3}\left(1,\{2, \ldots, n-1\} \Psi\left\{h_{j_{2}}, h_{j}, h_{1}\right\}, n ; \mathbf{h} /\left\{h_{1}, h_{j}, h_{j_{2}}\right\}\right)+\sum_{j_{3}=2, j_{3} \neq j, j_{2}}^{m}\left(f_{h_{j_{3}}} \cdot D_{h_{j} h_{j_{2}} h_{j_{3}}}\right)^{\mu} .
$$

Continuing the procedure until the coefficient $D$ does not contain any polarization vector $\epsilon$ (so the iteration stops), we obtain the complete recursive expansion of $A_{n, m}^{\mathrm{EYM}}$ as

$$
\begin{aligned}
A_{n, m}(1,2, \ldots, n ; \mathbf{h})= & \sum_{\Psi}\left(\epsilon_{h_{1}} \cdot Y_{h_{1}}\right) A_{n+1, m-1}\left(1,\{2, \ldots, n-1\} ш\left\{h_{1}\right\}, n ; \mathbf{h} /\left\{h_{1}\right\}\right) \\
& +\sum_{j=2}^{m}\left(\epsilon_{h_{1}} \cdot f_{h_{j}} \cdot Y_{h_{j}}\right) A_{n+2, m-2}\left(1,\{2, \ldots, n-1\} ш\left\{h_{j}, h_{1}\right\}, n ; \mathbf{h} /\left\{h_{1}, h_{j}\right\}\right) \\
& +\sum_{j=2}^{m} \sum_{j_{2}=2, j_{2} \neq j}^{m} \sum_{\Psi}\left(\epsilon_{h_{1}} \cdot f_{h_{j}} \cdot f_{h_{j_{2}}} \cdot Y_{h_{j_{2}}}\right) A_{n+3, m-3}\left(1,\{2, \ldots, n-1\} ш\left\{h_{j_{2}}, h_{j}, h_{1}\right\}, n ; \mathbf{h} /\left\{h_{1}, h_{j}, h_{j_{2}}\right\}\right) \\
& +\cdots \\
& +\sum_{j=2}^{m} \sum_{j_{2}=2, j_{2} \neq j}^{m} \ldots \sum_{j_{m} \neq j, j_{2}, \ldots, j_{m-1}} \sum_{\Psi}\left(\epsilon_{h_{1}} f_{h_{j}} \cdots f_{h_{j_{m}}} Y_{h_{j_{m}}}\right) A_{n+m}\left(1,\{2, \ldots, n-1\} ш\left\{h_{j_{m}}, h_{j_{m-1}}, \ldots, h_{1}\right\}, n\right) .
\end{aligned}
$$

In the above derivation, an important thing is that at each step, we need to show building blocks like $\left(\epsilon_{h_{1}} \cdot f_{h_{j}} \cdot f_{h_{j_{2}}} \cdot k_{h_{1}}\right),\left(\epsilon_{h_{1}} \cdot f_{h_{j}} \cdot f_{h_{j_{2}}} \cdot k_{h_{j}}\right)$, and $\left(\epsilon_{h_{1}} \cdot f_{h_{j}} \cdot\right.$ $\left.f_{h_{j_{2}}} \cdot k_{h_{j_{2}}}\right)$ with $j=2, \ldots, m$ having vanished coefficients. These building blocks belong to the "index circle structure" and in Appendix A we will prove their coefficients are zero.

Now we have reached our first goal, i.e., to expand sEYM amplitudes in the KK basis of YM amplitudes, by using differential operators. Compared to other methods appearing in the literature, our method provides a different angle. In the whole procedure, the use of building blocks is very crucial. By using them, we have translated the problem to the solving of linear algebraic equations. The solutions are naturally the KK basis of YM amplitudes. In other words, instead of assuming it, we have derived the fact that sEYM amplitudes can be expanded in terms of YM amplitudes.

\section{FURTHER APPLICATIONS}

As explained in the Introduction, the expansion of sEYM amplitudes in the KK basis of YM amplitudes is the primary example. In this section, we will demonstrate this claim by two new examples. In the first subsection, we will try to derive the expansion of gravity amplitudes using differential operators. Using the same strategy and results from the previous section, the expansion can be written down straightforwardly. In the second subsection, by using the observation that all other amplitudes can be obtained from acting on gravity amplitudes with proper differential operators [6,34], we derive the expansion of amplitudes of the Born-Infeld theory.

\section{A. Expansion of gravity amplitudes in the KK basis of YM amplitudes}

Let us consider the gravity amplitude $A^{\mathrm{G}}$ with $m$ gravitons. Expanding it according to the building blocks of $h_{1}$, we write

$$
A_{m}^{\mathrm{G}}\left(\left\{h_{i}\right\}\right)=\sum_{f=2}^{m-1}\left(\epsilon_{h_{1}} \cdot k_{h_{f}}\right)\left(\epsilon_{h_{f}} \cdot \mathcal{B}_{f}\right)+\sum_{g=2}^{m}\left(\epsilon_{h_{1}} \cdot \epsilon_{h_{g}}\right) \mathcal{D}_{g}
$$

where $k_{h_{m}}$ is eliminated using the momentum conservation. To determine $\mathcal{D}_{g}$, one can use the trace operator $\mathcal{T}_{h_{1} h_{q}}$, 
which turns the gravity amplitude to the sEYM amplitude with just two gluons. Applying it on the lhs of (4.1), it gives

$$
\mathcal{T}_{h_{1} h_{q}} A_{m}^{\mathrm{G}}\left(\left\{h_{i}\right\}\right)=A_{2, m-2}^{\mathrm{EYM}}\left(h_{q}, h_{1} ;\left\{h_{i}\right\} /\left\{h_{1}, h_{q}\right\}\right),
$$

while acting on the rhs it gives

$$
T_{h_{1} h_{q}}\left(\sum_{g=1}^{m}\left(\epsilon_{h_{1}} \cdot \epsilon_{h_{g}}\right) \mathcal{D}_{g}\right)=\mathcal{D}_{q} .
$$

Comparing two sides, we solve

$$
\mathcal{D}_{q}=A_{2, m-2}^{\mathrm{EYM}}\left(h_{q}, h_{1} ;\left\{h_{i}\right\} /\left\{h_{1}, h_{q}\right\}\right) .
$$

The vector $\mathcal{B}_{f}^{\mu}$ can be determined from $\mathcal{D}_{f}$ via the gauge invariance of graviton $h_{f}$. Let us consider the commutation relation

$$
\left[\mathcal{T}_{h_{f} h_{1} h_{m}}, \mathcal{W}_{h_{f}}\right]=\mathcal{T}_{h_{1} h_{f}} .
$$

Applying this relation on (4.1) gives

$-k_{h_{q}} \cdot \mathcal{B}_{q}=\mathcal{D}_{q}=A_{2, m-2}^{\mathrm{EYM}}\left(h_{q}, h_{1} ;\left\{h_{i}\right\} /\left\{h_{1}, h_{q}\right\}\right)$,

where the gauge invariant condition $\mathcal{W}_{h_{f}} A_{m}^{\mathrm{G}}\left(\left\{h_{i}\right\}\right)=0$ has been used. Thus we get ${ }^{12}$

$$
\begin{aligned}
A_{m}^{\mathrm{G}}\left(\left\{h_{i}\right\}\right)= & \sum_{f=2}^{m-1}\left(\epsilon_{h_{1}} \cdot f_{h_{f}} \cdot \mathcal{B}_{f}\right)+\left(\epsilon_{h_{1}} \cdot \epsilon_{h_{m}}\right) \\
& \times A_{2, m-2}^{\mathrm{EYM}}\left(h_{m}, h_{1} ;\left\{h_{i}\right\} /\left\{h_{1}, h_{m}\right\}\right) .
\end{aligned}
$$

To determine $\mathcal{B}_{f}^{\mu}$, s from the relations (4.6), we just need to find the expanded formula of sEYM amplitudes $A_{2, m-2}^{\mathrm{EYM}}\left(h_{q}, h_{1} ;\left\{h_{g}\right\} /\left\{h_{1}, h_{q}\right\}\right)$, which have been solved in the previous section using the differential operators and are given in (2.22). A special feature of the current situation is that there are no gluons $\{2, \ldots, r-1\}$ in (2.22) and we are left with only $\left\{\boldsymbol{h}, h_{a}\right\}$. Because of this fact, the coefficient given in (2.23) will always have $Y_{i_{s}}^{\mu}=k_{h_{q}}^{\mu}$ in our case, which is exactly the combination $-k_{h_{q}} \cdot \mathcal{B}_{q}$ in (4.6). In other words, using (2.22) and (2.23), after removing the $Y_{i_{s}}^{\mu}$, we get $B_{f}^{\mu}$ immediately.

It seems that the set of amplitudes that appeared in the above expansion is larger than the $\mathrm{KK}$ basis, since the appearance of the ordering $\left(h_{q}, \boldsymbol{h}, h_{a}, h_{1}\right)$ in (2.22). However, when we expand the sEYM amplitudes, the fiducial graviton $h_{a}$ can be chosen arbitrarily. Thus, we can always choose $h_{a}=h_{m}$ in (2.22). Then one can use the cyclic symmetry to rewrite any ordering $\left(h_{q}, \boldsymbol{h}, h_{m}, h_{1}\right)$ as

\footnotetext{
${ }^{12}$ We want to point out that the expansion form (4.7) has also appeared in [19] based on the Pfaffian expansion given in [43].
}

$\left(h_{1}, h_{q}, \boldsymbol{h}, h_{m}\right)$. On the other hand, the $\mathcal{D}_{m}$ term $A_{2, m-2}^{\mathrm{EYM}}\left(h_{m}, h_{1} ;\left\{h_{i}\right\} /\left\{h_{1}, h_{m}\right\}\right)$ equals to $A_{2, m-2}^{\mathrm{EYM}}\left(h_{1}, h_{m}\right.$; $\left.\left\{h_{i}\right\} /\left\{h_{1}, h_{m}\right\}\right)$. Thus, every amplitude appearing in the expansion has the ordering $\left(h_{1}, \ldots, h_{m}\right)$ and belongs to the chosen KK basis.

As a simple example, let us consider the expansion of three-graviton amplitude $A^{\mathrm{G}}\left(\left\{h_{1}, h_{2}, h_{3}\right\}\right)$. Using (4.7), (2.22), and (2.23) we get

$$
\begin{aligned}
A_{3}^{\mathrm{G}} & \left(\left\{h_{1}, h_{2}, h_{3}\right\}\right) \\
= & -\left(\epsilon_{h_{1}} \cdot f_{h_{2}} \cdot \epsilon_{h_{3}}\right) A_{3}^{\mathrm{YM}}\left(h_{2}, h_{3}, h_{1}\right) \\
& +\left(\epsilon_{h_{1}} \cdot \epsilon_{h_{3}}\right) A_{2,1}^{\mathrm{EYM}}\left(h_{3}, h_{1} ; h_{2}\right) \\
= & \left(\left(\epsilon_{h_{1}} \cdot \epsilon_{h_{3}}\right)\left(\epsilon_{h_{2}} \cdot k_{h_{1}}\right)-\left(\epsilon_{h_{1}} \cdot f_{h_{2}} \cdot \epsilon_{h_{3}}\right)\right) A_{3}^{\mathrm{YM}}\left(h_{1}, h_{2}, h_{3}\right) .
\end{aligned}
$$

The coefficient $\left(\left(\epsilon_{h_{1}} \cdot \epsilon_{h_{3}}\right)\left(\epsilon_{h_{2}} \cdot k_{h_{1}}\right)-\left(\epsilon_{h_{1}} \cdot f_{h_{2}} \cdot \epsilon_{h_{3}}\right)\right)$ is just the three-point YM amplitude $A_{3}^{\mathrm{YM}}\left(h_{1}, h_{2}, h_{3}\right)$; thus, the KLT relation $A_{3}^{\mathrm{G}}\left(\left\{h_{1}, h_{2}, h_{3}\right\}\right)=A_{3}^{\mathrm{YM}}\left(h_{1}, h_{2}, h_{3}\right)$ $A_{3}^{\mathrm{YM}}\left(h_{1}, h_{2}, h_{3}\right)$ has been reproduced.

\section{B. Expansion of Born-Infeld amplitudes in the KK basis of YM amplitudes}

As pointed out in [34], starting from the expansion of gravity amplitudes

$$
\begin{aligned}
A_{m}^{\mathrm{G}}(\{g\})= & \sum_{\varpi} C(ш) A^{\mathrm{YM}}(1,2 ш 3 ш \cdots ш(m-2) \\
& ш(m-1), m)
\end{aligned}
$$

expansions of amplitudes of different theories can be obtained by applying different combinations of differential operators on both sides of (4.9) simultaneously. The action of operators can be divided to two types. If the operators $\mathcal{O}^{\tilde{\varepsilon}}$ are defined via the polarization vectors $\tilde{\epsilon_{g}}$, both $A_{m}^{\mathrm{G}}(\{g\})$ and $A_{m}^{\mathrm{YM}}(1,2 ш 3 ш \cdots \omega(m-2) ш(m-1), m)$ will be transformed to amplitudes of other theories, while expansion coefficients are not modified. For example, one can use this procedure to expand YM amplitudes in terms of biadjoint scalar amplitudes, with the same coefficients. The second type is more interesting, where the operators $\mathcal{O}^{\epsilon}$ are defined via the polarization vectors $\epsilon_{g}$. While it turns the gravity amplitudes to amplitudes of other theories on the lhs, it modifies only expansion coefficients on the rhs. Using this observation we will expand amplitudes of the Born-Infeld (BI) theory in the KK basis of YM amplitudes in this subsection.

The $n$-point BI amplitudes can be created from the gravity ones by applying differential operators as

$$
A_{n}^{\mathrm{BI}}(\{g\})=\mathcal{L} \mathcal{T}_{a b} A_{n}^{\mathrm{G}}(\{g\}),
$$


where $\{g\}=\{1, \ldots, n\}$. The operator $\mathcal{L}$ is defined in $(2.30)$ as $\mathcal{L}=\prod_{i} \mathcal{L}_{i}$ with $i$ running over $\{g\} /\{a, b\}$ and $\mathcal{L}_{i} \equiv$ $\sum_{j}\left(k_{i} \cdot k_{j}\right) \partial_{\epsilon_{i} k_{j}}$ for $j \in\{g\} / i$. With the observation

$$
\mathcal{T}_{a b} A_{n}^{\mathrm{G}}(\{g\})=A_{2, n-2}^{\mathrm{EYM}}(a, b ;\{g\} /\{a, b\}),
$$

we have

$$
A_{n}^{\mathrm{BI}}(\{g\})=\mathcal{L} A_{2, n-2}^{\mathrm{EYM}}(a, b ;\{g\} /\{a, b\}) .
$$

Without loss of generality, let us choose $a=1$ and $b=n$. From the results in the previous subsection, we know how to expand the amplitude $A_{2, n-2}^{\mathrm{EYM}}(1, n ;\{g\} /\{1, n\})$ in terms of the YM ones as

$$
A_{2, n-2}^{\mathrm{EYM}}(1, n ;\{g\} /\{1, n\})=\sum_{ш} C(ш) A_{n}^{\mathrm{YM}}(1,2 ш 3 ш \cdots ш(n-1), n) .
$$

Applying the operator $\mathcal{L}$ on the lhs of (4.13) gives the BI amplitude $A_{n}^{\mathrm{BI}}(\{g\})$. For the rhs, $\mathcal{L}$ acts only on coefficients $C(w)$ since the YM amplitudes do not carry the polarization vectors $\epsilon$. The operator $\mathcal{L}$ turns all $\left(\epsilon_{i} \cdot k_{j}\right)$ 's to $\left(k_{i} \cdot k_{j}\right)$ 's; therefore, only terms with the form $\prod_{i}\left(\epsilon_{i} \cdot K_{i}\right)$ can survive under the action of $\mathcal{L}$ with $K_{i}$ 's being the sum of some external momenta. In $C_{n-2,2}(\omega)$, such a part is given by $\prod_{i}\left(\epsilon_{i} \cdot X_{i}\right)$. Carrying it out, the rhs gives

$$
\mathcal{L}\left(\sum_{ш} C(ш) A_{n}^{\mathrm{YM}}(1,2 ш 3 ш \cdots ш(n-1), n)\right)=\sum_{ш}\left(\prod_{i}\left(k_{i} \cdot X_{i}\right)\right) A_{n}^{\mathrm{YM}}(1,2 ш 3 ш \cdots ш(n-1), n) .
$$

Comparing the lhs with the rhs, the expansion of the BI amplitudes is obtained as

$$
A_{n}^{\mathrm{BI}}(\{g\})=\sum_{\varpi}\left(\prod_{i=2}^{n-1}\left(k_{i} \cdot X_{i}\right)\right) A_{n}^{\mathrm{YM}}(1,2 ш 3 ш \cdots ш(n-1), n)
$$

with the very compact expansion coefficients.

The expansion of BI amplitudes can also be derived via another definition of the operator $\mathcal{L}$,

$$
\mathcal{L}=\sum_{\rho \in \operatorname{pair}} \prod_{\{i, j\} \in \rho} \mathcal{L}_{i j}
$$

where $\mathcal{L}_{i j} \equiv-\left(k_{i} \cdot k_{j}\right) \partial_{\epsilon_{i} \epsilon_{j}}$ and the pairs $\{i, j\}$ run over the set $\{g\} /\{1, n\}$. Applying this definition on the rhs of (4.13), the survived terms are those in which each polarization vector $\epsilon_{i}$ is contracted with another polarization vector $\epsilon_{j}$. Using the results (2.22) and (2.23), such a part is found to be

$$
\sum_{\left|\mathrm{Or}_{k}\right|_{\text {even }}} \sum_{w}\left(\prod_{k=1}^{t}(-)^{\frac{\left|\mathrm{Or}_{k}\right|}{2}} M_{k}(ш)\right) A_{n}^{\mathrm{YM}}\left(1, \mathrm{Or}_{1} ш \mathrm{Or}_{2} ш \cdots ш \mathrm{Or}_{t}, n\right)
$$

where $\mathrm{Or}_{1} \cup \mathrm{Or}_{2} \cup \cdots \mathrm{Or}_{t}=\{2, \ldots, n-1\}$, and the summation $\sum_{\left|\mathrm{Or}_{k}\right|_{\text {even }}}$ is over all possible ordered splitting that the lengths of all subsets are even. Furthermore, $M_{k}(\omega)$ for the length- $r$ subset $\mathrm{Or}_{k}=\left\{\gamma_{1}, \gamma_{2}, \cdots \gamma_{r}\right\}$ is defined as ${ }^{13}$

$$
M_{k}(\omega)=\left(\epsilon_{\gamma_{r}} \cdot \epsilon_{\gamma_{r-1}}\right)\left(k_{\gamma_{r-1}} \cdot k_{\gamma_{r-2}}\right)\left(\epsilon_{\gamma_{r-2}} \cdot \epsilon_{\gamma_{r-3}}\right) \cdots\left(k_{\gamma_{3}} \cdot k_{\gamma_{2}}\right)\left(\epsilon_{\gamma_{2}} \cdot \epsilon_{\gamma_{1}}\right)\left(k_{\gamma_{1}} \cdot Z_{\gamma_{1}}\right) .
$$

Acting $\mathcal{L}$ on $M_{k}$, it gives

$$
N_{k}(\Psi)=(-)^{\frac{\left|\mathrm{O}_{k}\right|}{2}}\left(k_{\gamma_{r}} \cdot k_{\gamma_{r-1}}\right)\left(k_{\gamma_{r-1}} \cdot k_{\gamma_{r-2}}\right)\left(k_{\gamma_{r-2}} \cdot k_{\gamma_{r-3}}\right) \cdots\left(k_{\gamma_{3}} \cdot k_{\gamma_{2}}\right)\left(k_{\gamma_{2}} \cdot k_{\gamma_{1}}\right)\left(k_{\gamma_{1}} \cdot Z_{\gamma_{1}}\right)
$$

Then we get

\footnotetext{
${ }^{13}$ The definition of $Z_{i}$ can be found in [19]. Since it has not been used much in this paper, we have not reviewed it in Sec. II.
} 


$$
A_{n}^{\mathrm{BI}}(\{g\})=\sum_{\left|\mathrm{Or}_{k}\right|_{\text {even }}} \sum_{\Psi}\left(\prod_{k=1}^{t} N_{k}(ш)\right) A_{n}^{\mathrm{YM}}\left(1, \mathrm{Or}_{1} ш \mathrm{Or}_{2} ш \cdots ш \mathrm{Or}_{t}, n\right) .
$$

These two expressions (4.15) and (4.20) should be equal if both are correct. Let us verify such an equality for the simplest four-point example. For the four-point case, (4.15) gives

$$
A_{4}^{\mathrm{BI}}(\{1,2,3,4\})=\left(k_{2} \cdot k_{1}\right)\left(k_{3} \cdot\left(k_{1}+k_{2}\right)\right) A_{4}^{\mathrm{YM}}(1,2,3,4)+\left(k_{2} \cdot\left(k_{1}+k_{3}\right)\right)\left(k_{3} \cdot k_{1}\right) A_{4}^{\mathrm{YM}}(1,3,2,4),
$$

while (4.20) gives

$$
A_{4}^{\mathrm{BI}}(\{1,2,3,4\})=\left(k_{2} \cdot k_{3}\right)\left(k_{3} \cdot k_{1}\right) A_{4}^{\mathrm{YM}}(1,3,2,4),
$$

with the chosen order $2 \prec 3$. These two results are equal to each other only after using BCJ relations (2.13). The example shows that although (4.20) looks more complicated, it gives shorter expressions. As an interesting remark, if we reverse the logic, i.e., assuming the equivalence between two differential operators, we will arrive at the equivalence of results (4.15) and (4.20). From them, we can derive the generalized fundamental BCJ relations (2.9).

\section{EXPANSION OF SEYM AMPLITUDES IN THE BCJ BASIS OF YM AMPLITUDES}

As indicated in the Introduction, the expansion of sEYM amplitudes in terms of YM amplitudes can be divided into two types: the expansion in the KK basis and the expansion in the BCJ basis. Up to now, expansion in the literature is in the KK basis, where the expansion coefficients are much simpler. In this paper, we will initiate the investigation of expanding in the $\mathrm{BCJ}$ basis. There are two different approaches we could think of. In the first approach, we start from the expansion in the KK basis (2.22) and then transform the KK basis to the BCJ basis using (2.13). This approach is straightforward, but as shown in this section, the algebraic manipulations are not so trivial. In the second approach, encouraged by results in the previous sections, we will use differential operators. Both approaches will be explored in this paper. In this section, we will take the first approach, while in the next section, we will take the second approach. As emphasized in the Introduction, we are looking for a more efficient method to find the expansion coefficients, which now will be a more complicated rational function. Results obtained in this section will be compared with results found in the next section.

\section{A. Case with one graviton}

We start with the sEYM amplitudes with just a graviton. The expansion in the KK basis is given by

$$
A_{n, 1}^{\mathrm{EYM}}(1,2, \ldots, n ; p)=\sum_{ш}\left(\epsilon_{p} \cdot Y_{p}\right) A_{n+1}^{\mathrm{YM}}(1,\{2, \ldots, n-1\} ш\{p\}, n) .
$$

To expand in the BCJ basis, we use a trick, i.e., the gauge invariance of $p$, so we have

$$
0=\left(k_{p} \cdot Y_{p}\right) A_{n+1}^{\mathrm{YM}}(1,2,\{3, \ldots, n-1\} ш\{p\}, n)+\left(k_{p} \cdot k_{1}\right) A_{n+1}^{\mathrm{YM}}(1, p,\{2, \ldots, n-1\}, n) .
$$

Solving the equation and putting it back, we have

$$
\begin{aligned}
A_{n, 1}^{\mathrm{EYM}}(1,2, \ldots, n ; p) & =\left(\epsilon_{p} \cdot Y_{p}\right) A_{n+1}^{\mathrm{YM}}(1,2,\{3, \ldots, n-1\} w\{p\}, n)-\left(\epsilon_{p} \cdot k_{1}\right) \frac{\left(k_{p} \cdot Y_{p}\right)}{\left(k_{p} \cdot k_{1}\right)} A_{n+1}^{\mathrm{YM}}(1,2,\{3, \ldots, n-1\} w\{p\}, n) \\
& =\frac{\left(\epsilon_{p} \cdot Y_{p}\right)\left(k_{p} \cdot k_{1}\right)-\left(\epsilon_{p} \cdot k_{1}\right)\left(k_{p} \cdot Y_{p}\right)}{\left(k_{p} \cdot k_{1}\right)} A_{n+1}^{\mathrm{YM}}(1,2,\{3, \ldots, n-1\} ш\{p\}, n) .
\end{aligned}
$$

Using the definition of field strength, we arrive

$$
A_{n, 1}^{\mathrm{EYM}}(1,2, \ldots, n ; p)=\frac{k_{1} \cdot f_{p} \cdot Y_{p}}{\left(k_{p} \cdot k_{1}\right)} A_{n+1}^{\mathrm{YM}}(1,2,\{3, \ldots, n-1\} ш\{p\}, n) .
$$


The expansion coefficients are rational functions with pole $\left(k_{p} \cdot k_{1}\right)$, which is crucial to match up the structure of physical poles at both sides. The observation implies that the expansion coefficients in the $\mathrm{BCJ}$ basis will be, in general, the rational functions with proper pole structures.

\section{B. Case with two gravitons}

Using the recursive expansion (2.23), we could write down

$$
\begin{aligned}
A_{n, 2}^{\mathrm{EYM}}(1,2, \ldots, n ; p, q)= & \sum_{\Psi}\left(\epsilon_{p} \cdot Y_{p}\right) A_{n+1,1}^{\mathrm{EYM}}(1,\{2, \ldots, n-1\} ш\{p\}, n ; q) \\
& +\sum_{\Psi}\left(\epsilon_{p} \cdot f_{q} \cdot Y_{q}\right) A_{n+2}^{\mathrm{YM}}(1,\{2, \ldots, n-1\} ш\{q, p\}, n) .
\end{aligned}
$$

The gauge invariance of $p$ leads to the following equation:

$$
\begin{aligned}
0= & \left(k_{p} \cdot Y_{p}\right) A_{n+1,1}^{\mathrm{EYM}}(1,\{2, \ldots, n-1\} ш\{p\}, n ; q)+\left(k_{p} \cdot f_{q} \cdot Y_{q}\right) A_{n+2}^{\mathrm{YM}}(1,\{2, \ldots, n-1\} ш\{q, p\}, n) \\
= & \left(k_{p} \cdot Y_{p}\right) A_{n+1,1}^{\mathrm{EYM}}(1,2,\{3, \ldots, n-1\} ш\{p\}, n ; q)+\left(k_{p} \cdot k_{1}\right) A_{n+1,1}^{\mathrm{EYM}}(1, p,\{2, \ldots, n-1\}, n ; q) \\
& +\left(k_{p} \cdot f_{q} \cdot Y_{q}\right) A_{n+2}^{\mathrm{YM}}(1,\{2, \ldots, n-1\} ш\{q, p\}, n) .
\end{aligned}
$$

Solving the $A_{n+1,1}^{\mathrm{EYM}}(1, p,\{2, \ldots, n-1\}, n ; q)$ and putting it back, we get

$$
\begin{aligned}
A_{n, 2}^{\mathrm{EYM}}(1,2, \ldots, n ; p, q)= & \frac{\left(k_{1} \cdot f_{p} \cdot Y_{p}\right)}{\mathcal{K}_{1 p}} A_{n+1,1}^{\mathrm{EYM}}(1,2,\{3, \ldots, n-1\} ш\{p\}, n ; q) \\
& +\frac{\left(k_{1} \cdot f_{p} \cdot f_{q} \cdot Y_{q}\right)}{\mathcal{K}_{1 p}} A_{n+2}^{\mathrm{YM}}(1,\{2, \ldots, n-1\} ш\{q, p\}, n) .
\end{aligned}
$$

Although we have not reached the expansion in the BCJ basis, the gauge invariance of all gravitons is manifest due to the only appearance of $f_{p}, f_{q}$.

For the first term of (5.6), we use the result (5.4) to reach

$$
T_{1}=\frac{\left(k_{1} \cdot f_{p} \cdot Y_{p}\right)}{\mathcal{K}_{1 p}} \frac{\left(k_{1} \cdot f_{q} \cdot X_{q}\right)}{\mathcal{K}_{1 q}} A_{n+2}^{\mathrm{YM}}(1,2,\{\{3, \ldots, n-1\} ш\{p\}\} ш\{q\}, n),
$$

where it is important to notice that since for $q$, the $p$ is just a gluon, thus we must use the $X_{q}$ instead of the $Y_{q}$. Expanding further to different orderings, we get

$$
\begin{aligned}
T_{1}= & \frac{\left(k_{1} \cdot f_{p} \cdot Y_{p}\right)}{\mathcal{K}_{1 p}} \frac{\left(k_{1} \cdot f_{q} \cdot Y_{q}\right)}{\mathcal{K}_{1 q}} A_{n+2}^{\mathrm{YM}}(1,2,\{3, \ldots, n-1\} ш\{q, p\}, n) \\
& +\frac{\left(k_{1} \cdot f_{p} \cdot Y_{p}\right)}{\mathcal{K}_{1 p}} \frac{\left(k_{1} \cdot f_{q} \cdot\left(Y_{q}+k_{p}\right)\right)}{\mathcal{K}_{1 q}} A_{n+2}^{\mathrm{YM}}(1,2,\{3, \ldots, n-1\} ш\{p, q\}, n) .
\end{aligned}
$$

For the second term in (5.6), we split it to

$$
\begin{aligned}
T_{2}= & \frac{\left(k_{1} \cdot f_{p} \cdot f_{q} \cdot k_{1}\right)}{\mathcal{K}_{1 p}} A_{n+2}^{\mathrm{YM}}(1, q, p,\{2, \ldots, n-1\}, n)+\frac{\left(k_{1} \cdot f_{p} \cdot f_{q} \cdot k_{1}\right)}{\mathcal{K}_{1 p}} A_{n+2}^{\mathrm{YM}}(1, q, 2,\{3, \ldots, n-1\} ш\{p\}, n) \\
& +\frac{\left(k_{1} \cdot f_{p} \cdot f_{q} \cdot Y_{q}\right)}{\mathcal{K}_{1 p}} A_{n+2}^{\mathrm{YM}}(1,2,\{3, \ldots, n-1\} ш\{q, p\}, n) .
\end{aligned}
$$

For the first term of $T_{2}$, we use the result (2.11) to get 


$$
\begin{aligned}
T_{2,1}= & \frac{\left(k_{1} \cdot f_{p} \cdot f_{q} \cdot k_{1}\right)}{\mathcal{K}_{1 p}}\left\{\frac{\left(k_{q} \cdot k_{1}+k_{p} \cdot\left(Y_{p}+k_{q}\right)\right)\left(k_{q} \cdot\left(Y_{q}+k_{p}\right)\right)}{\mathcal{K}_{1 p q} \mathcal{K}_{1 q}} A_{n+1}^{\mathrm{YM}}(1,2,\{3, \ldots, n-1\} ш\{p, q\}, n)\right. \\
& \left.+\frac{\left(k_{q} \cdot\left(Y_{q}-k_{1}\right)\right)\left(k_{p} \cdot\left(Y_{p}+k_{q}\right)\right)}{\mathcal{K}_{1 p q} \mathcal{K}_{1 q}} A_{n+2}^{\mathrm{YM}}(1,2,\{3, \ldots, n-1\} ш\{q, p\}, n)\right\} .
\end{aligned}
$$

For the second term of $T_{2}$, we use (2.10) to get

$$
\begin{aligned}
T_{2,2}= & \frac{\left(k_{1} \cdot f_{p} \cdot f_{q} \cdot k_{1}\right)}{\mathcal{K}_{1 p}} \frac{-\left(k_{q} \cdot X_{q}\right)}{\left(k_{q} \cdot k_{1}\right)} A_{n+2}^{\mathrm{YM}}(1,2,\{\{3, \ldots, n-1\} ш\{p\}\} ш\{q\}, n) \\
= & -\frac{\left(k_{1} \cdot f_{p} \cdot f_{q} \cdot k_{1}\right)}{\mathcal{K}_{1 p}} \frac{\left(k_{q} \cdot Y_{q}\right)}{\left(k_{q} \cdot k_{1}\right)} A_{n+2}^{\mathrm{YM}}(1,2,\{3, \ldots, n-1\} ш\{q, p\}, n) \\
& -\frac{\left(k_{1} \cdot f_{p} \cdot f_{q} \cdot k_{1}\right)}{\mathcal{K}_{1 p}} \frac{\left(k_{q} \cdot\left(Y_{q}+k_{p}\right)\right)}{\left(k_{q} \cdot k_{1}\right)} A_{n+2}^{\mathrm{YM}}(1,2,\{3, \ldots, n-1\} ш\{p, q\}, n) .
\end{aligned}
$$

Putting $T_{2,1}, T_{2,2}$ back, we get

$$
\begin{aligned}
T_{2}= & \frac{\left(k_{1} \cdot f_{p} \cdot f_{q} \cdot k_{1}\right)\left[\left(k_{q} \cdot\left(Y_{q}-k_{1}\right)\right)\left(k_{p} \cdot\left(Y_{p}+k_{q}\right)\right)-\left(k_{q} \cdot Y_{q}\right) \mathcal{K}_{1 p q}\right]}{\mathcal{K}_{1 p q} \mathcal{K}_{1 q} \mathcal{K}_{1 p}} A_{n+2}^{\mathrm{YM}}(1,2,\{3, \ldots, n-1\} ш\{q, p\}, n) \\
& +\frac{\left(k_{1} \cdot f_{p} \cdot f_{q} \cdot k_{1}\right)\left(k_{p} \cdot\left(Y_{p}-k_{1}\right)\right)\left(k_{q} \cdot\left(Y_{q}+k_{p}\right)\right)}{\mathcal{K}_{1 p q} \mathcal{K}_{1 q} \mathcal{K}_{1 p}} A_{n+2}^{\mathrm{YM}}(1,2,\{3, \ldots, n-1\} ш\{p, q\}, n) \\
& +\frac{\left(k_{1} \cdot f_{p} \cdot f_{q} \cdot Y_{q}\right)}{\mathcal{K}_{1 p}} A_{n+2}^{\mathrm{YM}}(1,2,\{3, \ldots, n-1\} w\{q, p\}, n) .
\end{aligned}
$$

Adding $T_{1}, T_{2}$ together, we have

$$
\begin{aligned}
A_{n, 2}^{\mathrm{EYM}}(1,2, \ldots, n ; p, q)= & \sum_{\varpi} C(\{p, q\}, ш) A_{n+2}^{\mathrm{YM}}(1,2,\{3, \ldots, n-1\} ш\{p, q\}, n) \\
& +\sum_{\varpi} C(\{q, p\}, ш) A_{n+2}^{\mathrm{YM}}(1,2,\{3, \ldots, n-1\} ш\{q, p\}, n)
\end{aligned}
$$

with coefficients

$$
\begin{aligned}
C(\{p, q\}, ш)= & \left\{\frac{\left(k_{1} \cdot f_{p} \cdot Y_{p}\right)}{\mathcal{K}_{1 p}} \frac{\left(k_{1} \cdot f_{q} \cdot\left(Y_{q}+k_{p}\right)\right)}{\mathcal{K}_{1 q}}+\frac{\left(k_{1} \cdot f_{p} \cdot f_{q} \cdot k_{1}\right)\left(k_{p} \cdot\left(Y_{p}-k_{1}\right)\right)\left(k_{q} \cdot\left(Y_{q}+k_{p}\right)\right)}{\mathcal{K}_{1 p q} \mathcal{K}_{1 q} \mathcal{K}_{1 p}}\right\} \\
C(\{q, p\}, ш)= & \left\{\frac{\left(k_{1} \cdot f_{p} \cdot Y_{p}\right)}{\mathcal{K}_{1 p}} \frac{\left(k_{1} \cdot f_{q} \cdot Y_{q}\right)}{\mathcal{K}_{1 q}}+\frac{\left(k_{1} \cdot f_{p} \cdot f_{q} \cdot Y_{q}\right)}{\mathcal{K}_{1 p}}\right. \\
& \left.+\frac{\left(k_{1} \cdot f_{p} \cdot f_{q} \cdot k_{1}\right)\left[\left(k_{q} \cdot\left(Y_{q}-k_{1}\right)\right)\left(k_{p} \cdot\left(Y_{p}+k_{q}\right)\right)-\left(k_{q} \cdot Y_{q}\right) \mathcal{K}_{1 p q}\right]}{\mathcal{K}_{1 p q} \mathcal{K}_{1 q} \mathcal{K}_{1 p}}\right\} .
\end{aligned}
$$

The first coefficient can be rewritten as

$$
C(\{p, q\}, \omega)=\left\{\frac{\left(k_{1} \cdot f_{p} \cdot X_{p}\right)}{\mathcal{K}_{1 p}} \frac{\left(k_{1} \cdot f_{q} \cdot X_{q}\right)}{\mathcal{K}_{1 q}}+\frac{\left(k_{1} \cdot f_{p} \cdot f_{q} \cdot k_{1}\right)\left(k_{p} \cdot\left(Y_{p}-k_{1}\right)\right)\left(k_{q} \cdot X_{q}\right)}{\mathcal{K}_{1 p q} \mathcal{K}_{1 q} \mathcal{K}_{1 p}}\right\} .
$$

When comparing the factor $\frac{\left(k_{p} \cdot\left(Y_{p}-k_{1}\right)\right)\left(k_{q} \cdot X_{q}\right)}{\mathcal{K}_{1 p q} \mathcal{K}_{1 p}}$ in the second term of $C(\{p, q\}, w)$ with the second term of $(2.11)$, we see they are the same. It is not a coincidence and we will see its reasoning in the next section. The second coefficient $C(\{q, p\}$, $ш)$ is not related to the first coefficient by permutation at the current form; thus, we need to do some manipulations. It is easy to see that the first two terms can be rewritten as 


$$
\frac{\left(k_{1} \cdot f_{p} \cdot X_{p}\right)}{\mathcal{K}_{1 p}} \frac{\left(k_{1} \cdot f_{q} \cdot Y_{q}\right)}{\mathcal{K}_{1 q}}+\frac{\left(k_{1} \cdot f_{p} \cdot f_{q} \cdot k_{1}\right)\left(Y_{q} \cdot k_{q}\right)}{\mathcal{K}_{1 p} \mathcal{K}_{1 q}}
$$

where we have used the identity (5.14)

$$
\left(B \cdot f_{p} \cdot k_{1}\right)\left(k_{q} \cdot k_{p}\right)=\left(B \cdot f_{p} \cdot k_{q}\right)\left(k_{1} \cdot k_{p}\right)+\left(k_{q} \cdot f_{p} \cdot k_{1}\right)\left(B \cdot k_{p}\right) .
$$

The identity (5.14) can be easily remembered as the following. ${ }^{14}$ The two elements $B, k_{1}$ in $\left(B \cdot f_{p} \cdot k_{1}\right)$ are exchanged with the element $k_{q}$ in $\left(k_{q} \cdot k_{p}\right)$. Putting it back, the second coefficient becomes

$$
C(\{q, p\}, ш)=\frac{\left(k_{1} \cdot f_{p} \cdot X_{p}\right)}{\mathcal{K}_{1 p}} \frac{\left(k_{1} \cdot f_{q} \cdot Y_{q}\right)}{\mathcal{K}_{1 q}}+\frac{\left(k_{1} \cdot f_{p} \cdot f_{q} \cdot k_{1}\right)\left(k_{q} \cdot\left(Y_{q}-k_{1}\right)\right)\left(k_{p} \cdot\left(Y_{p}+k_{q}\right)\right)}{\mathcal{K}_{1 p q} \mathcal{K}_{1 q} \mathcal{K}_{1 p}}
$$

Now the permutation relation with $C(\{p, q\}, w)$ is manifest.

Before ending this subsection, we give some remarks for the above results:

(1) First, the two coefficients (5.13) and (5.15) are related to each other by a permutation as it should be. This relation can also be used as a consistent check of our calculations.

(2) Each coefficient contains two terms. The first term is the product of two factors $\frac{k_{1} f_{i} X_{i}}{\mathcal{K}_{1 i}}$ with $i=p, q$. The same factor has also appeared in (5.4) for the case having just one graviton, and can be interpreted as "turning a graviton to a gluon in the BCJ basis" in a loose sense. The second term has the factor $\left(k_{1} f_{p} f_{q} k_{1}\right)$, which contains the contraction $\epsilon_{p} \cdot \epsilon_{q}$ as a signal of the mutual interaction. Thus, the physical picture of these two terms is clear.

(3) One important observation is that the factor $\left(k_{1} f_{p} f_{q} k_{1}\right)$ appears naturally in $C(\{p, q\}$, $)$, but not in $C(\{q, p\}, ш)$. Only with some manipulations can we transfer the form $\left(k_{1} f_{p} f_{q} Y_{q}\right)$ to $\left(k_{1} f_{p} f_{q} k_{1}\right)$. As we will see for the case with three gravitons, the same pattern will appear again. In other words, the contraction of $\epsilon \cdot \epsilon$ can always be included inside the form $k_{1} f \ldots f k_{1}$. Observations given in the second and third points provide information of the building blocks for expansion in the BCJ basis when using differential operators.

(4) Although it is straightforward, the whole calculation is nontrivial. An indication is that in the middle steps, the BCJ coefficients (2.13) have been used many times; thus, expressions in middle steps are lengthy and complicated. However, when summing them up, cancellations happen and the final result (5.13) is very simple. Similar phenomena have been met many times in the history of scattering amplitudes. Thus, we believe that there should be a more efficient method where cancellations in middle steps are automatically avoided.

\section{The case of three gravitons}

Using the recursive expansion (2.23), the expansion in the KK basis is given by

$$
\begin{aligned}
A_{n, 3}^{\mathrm{EYM}}(1,2, \ldots, n ; p, q, r)= & \left(\epsilon_{p} \cdot Y_{p}\right) A_{n+1,2}^{\mathrm{EYM}}(1,\{2, \ldots, n-1\} ш\{p\}, n ; q, r) \\
& +\left(\epsilon_{p} \cdot f_{q} \cdot Y_{q}\right) A_{n+2,1}^{\mathrm{EYM}}(1,\{2, \ldots, n-1\} ш\{q, p\}, n ; r) \\
& +\left(\epsilon_{p} \cdot f_{r} \cdot Y_{r}\right) A_{n+2,1}^{\mathrm{EYM}}(1,\{2, \ldots, n-1\} ш\{r, p\}, n ; q) \\
& +\left(\epsilon_{p} \cdot f_{q} \cdot f_{r} \cdot Y_{r}\right) A_{n+3}^{\mathrm{YM}}(1,\{2, \ldots, n-1\} ш\{r, q, p\}, n) \\
& +\left(\epsilon_{p} \cdot f_{r} \cdot f_{q} \cdot Y_{q}\right) A_{n+3}^{\mathrm{YM}}(1,\{2, \ldots, n-1\} ш\{q, r, p\}, n) .
\end{aligned}
$$

By the same trick, i.e., the gauge invariance of $p$, we can solve $A_{n+1,2}^{\mathrm{EYM}}(1, p,\{2,3, \ldots, n-1\}, n ; q, r)$. After putting it back to (5.16) and simplifying, we reach

\footnotetext{
${ }^{14}$ The formula is also like the Schouten identity $\langle a b\rangle\langle c d\rangle=\langle a c\rangle\langle b d\rangle+\langle a d\rangle\langle c b\rangle$.
} 


$$
\begin{aligned}
A_{n, 3}^{\mathrm{EYM}}(1,2, \ldots, n ; p, q, r)= & \frac{\left(k_{1} \cdot f_{p} \cdot Y_{p}\right)}{\left(k_{p} \cdot k_{1}\right)} A_{n+1,2}^{\mathrm{EYM}}(1,2,\{3, \ldots, n-1\} ш\{p\}, n ; q, r) \\
& +\frac{\left(k_{1} \cdot f_{p} \cdot f_{q} \cdot Y_{q}\right)}{\left(k_{p} \cdot k_{1}\right)} A_{n+2,1}^{\mathrm{EYM}}(1,\{2, \ldots, n-1\} ш\{q, p\}, n ; r) \\
& +\frac{\left(k_{1} \cdot f_{p} \cdot f_{r} \cdot Y_{r}\right)}{\left(k_{p} \cdot k_{1}\right)} A_{n+2,1}^{\mathrm{EYM}}(1,\{2, \ldots, n-1\} ш\{r, p\}, n ; q) \\
& +\frac{\left(k_{1} \cdot f_{p} \cdot f_{q} \cdot f_{r} \cdot Y_{r}\right)}{\left(k_{p} \cdot k_{1}\right)} A_{n+3}^{\mathrm{YM}}(1,\{2, \ldots, n-1\} \omega\{r, q, p\}, n) \\
& +\frac{\left(k_{1} \cdot f_{p} \cdot f_{r} \cdot f_{q} \cdot Y_{q}\right)}{\left(k_{p} \cdot k_{1}\right)} A_{n+3}^{\mathrm{YM}}(1,\{2, \ldots, n-1\} ш\{q, r, p\}, n),
\end{aligned}
$$

where since only $f$ 's appear, the gauge invariance of all gravitons is manifest. To expand in the BCJ basis, i.e.,

$$
A_{n, 3}^{\mathrm{EYM}}(1,2, \ldots, n ; p, q, r)=\sum_{\rho \in S_{3}} \sum_{ш} C(\rho, ш) A_{n+2}^{\mathrm{YM}}\left(1,2,\{3, \ldots, n-1\} ш\left\{\rho_{1}, \rho_{2}, \rho_{3}\right\}, n\right)
$$

with $\rho=\left\{\rho_{1}, \rho_{2}, \rho_{3}\right\}=\rho\{p, q, r\}$ the permutation of $\{p, q, r\}$, we need to expand each term in (5.17) in that basis. Now we do it term by term.

The first term: For the first term, we can use the result (5.11) directly, only paying attention to the meaning of $Y_{q}, Y_{r}$ since for $q, r$, the $p$ likes a gluon. Thus, we have

$$
\begin{aligned}
T_{1}= & \frac{\left(k_{1} \cdot f_{p} \cdot Y_{p}\right)}{\left(k_{p} \cdot k_{1}\right)} A_{n+3}^{\mathrm{YM}}(1,2,\{\{3, \ldots, n-1\} w\{p\}\} w\{r, q\}, n) \\
& \times\left\{\frac{\left(k_{1} \cdot f_{r} \cdot X_{r}\right)}{\mathcal{K}_{1 r}} \frac{\left(k_{1} \cdot f_{q} \cdot X_{q}\right)}{\mathcal{K}_{1 q}}+\frac{\left(k_{1} \cdot f_{r} \cdot f_{q} \cdot k_{1}\right)\left(k_{r} \cdot\left(X_{r}-k_{1}\right)\right)\left(k_{q} \cdot X_{q}\right)}{\mathcal{K}_{1 q r} \mathcal{K}_{1 q} \mathcal{K}_{1 r}}\right\} \\
& +\frac{\left(k_{1} \cdot f_{p} \cdot Y_{p}\right)}{\left(k_{p} \cdot k_{1}\right)} A_{n+3}^{\mathrm{YM}}(1,2,\{\{3, \ldots, n-1\} w\{p\}\} w\{q, r\}, n) \\
& \times\left\{\frac{\left(k_{1} \cdot f_{r} \cdot X_{r}\right)}{\mathcal{K}_{1 r}} \frac{\left(k_{1} \cdot f_{q} \cdot X_{q}\right)}{\mathcal{K}_{1 q}}+\frac{\left(k_{1} \cdot f_{q} \cdot f_{r} \cdot k_{1}\right)\left(k_{q} \cdot\left(X_{q}-k_{1}\right)\right)\left(k_{r} \cdot X_{r}\right)}{\mathcal{K}_{1 q r} \mathcal{K}_{1 q} \mathcal{K}_{1 r}}\right\} .
\end{aligned}
$$

Now we can find contributions to six coefficients in (5.18). Since we expect they are related to each other by permutations, we will only write down contributions to the coefficient $C(\{p, q, r\})$ for simplicity ${ }^{15}$

$$
\begin{aligned}
C^{T_{1}}(\{p, q, r\})= & \frac{\left(k_{1} \cdot f_{p} \cdot Y_{p}\right)}{\left(k_{p} \cdot k_{1}\right)} \frac{\left(k_{1} \cdot f_{r} \cdot\left(Y_{r}+k_{p}+k_{q}\right)\right)}{\mathcal{K}_{1 r}} \frac{\left(k_{1} \cdot f_{q} \cdot\left(Y_{q}+k_{p}\right)\right)}{\mathcal{K}_{1 q}} \\
& +\frac{\left(k_{1} \cdot f_{p} \cdot Y_{p}\right)}{\left(k_{p} \cdot k_{1}\right)} \frac{\left(k_{1} \cdot f_{q} \cdot f_{r} \cdot k_{1}\right)\left(k_{q} \cdot\left(Y_{q}+k_{p}-k_{1}\right)\right)\left(k_{r} \cdot\left(Y_{r}+k_{p}+k_{q}\right)\right)}{\mathcal{K}_{1 q r} \mathcal{K}_{1 q} \mathcal{K}_{1 r}}
\end{aligned}
$$

The second term: The second term can be split to

$$
\begin{aligned}
T_{2}= & \frac{\left(k_{1} \cdot f_{p} \cdot f_{q} \cdot Y_{q}\right)}{\left(k_{p} \cdot k_{1}\right)} A_{n+2,1}^{\mathrm{EYM}}(1,2,\{3, \ldots, n-1\} ш\{q, p\}, n ; r) \\
& +\frac{\left(k_{1} \cdot f_{p} \cdot f_{q} \cdot k_{1}\right)}{\left(k_{p} \cdot k_{1}\right)} A_{n+2,1}^{\mathrm{EYM}}(1, q,\{2,3, \ldots, n-1\} ш\{p\}, n ; r) .
\end{aligned}
$$

Among these two terms, using the result (5.4), the first term can be expanded to

\footnotetext{
${ }^{15}$ As pointed out in the previous subsection, for a given choice of fiducial graviton in the KK-basis expansion, some orderings will have simpler expressions for coefficients. This is the reason we consider this ordering.
} 


$$
T_{2,1}=\frac{\left(k_{1} \cdot f_{p} \cdot f_{q} \cdot Y_{q}\right)}{\mathcal{K}_{1 p}} \frac{\left(k_{1} \cdot f_{r} \cdot X_{r}\right)}{\mathcal{K}_{1 r}} A_{n+3}^{\mathrm{YM}}(1,2,\{\{3, \ldots, n-1\} ш\{q, p\}\} ш\{r\}, n) .
$$

It is important to notice that this term does not contribute to the coefficient $C(\{p, q, r\})$; thus, we can forget it at this moment. ${ }^{16}$ The second term can be expanded to

$$
\begin{aligned}
T_{2,2}= & \frac{\left(k_{1} \cdot f_{p} \cdot f_{q} \cdot k_{1}\right)}{\left(k_{p} \cdot k_{1}\right)} \frac{\left(k_{1} \cdot f_{r} \cdot X_{r}\right)}{\mathcal{K}_{1 r}} A_{n+3}^{\mathrm{YM}}(1, q,\{2,3, \ldots, n-1\} \omega\{p\} \omega\{r\}, n) \\
= & \frac{\left(k_{1} \cdot f_{p} \cdot f_{q} \cdot k_{1}\right)}{\left(k_{p} \cdot k_{1}\right)} \frac{\left(k_{1} \cdot f_{r} \cdot\left(Y_{r}+k_{q}+k_{p}\right)\right)}{\mathcal{K}_{1 r}} A_{n+3}^{\mathrm{YM}}(1, q,\{2,3, \ldots, n-1\} ш\{p, r\}, n) \\
& +\frac{\left(k_{1} \cdot f_{p} \cdot f_{q} \cdot k_{1}\right)}{\left(k_{p} \cdot k_{1}\right)} \frac{\left(k_{1} \cdot f_{r} \cdot\left(Y_{r}+k_{q}\right)\right)}{\mathcal{K}_{1 r}} A_{n+3}^{\mathrm{YM}}(1, q,\{2,3, \ldots, n-1\} ш\{r, p\}, n),
\end{aligned}
$$

where to distinguish different situations, it is crucial to expand $X_{r}$ to $Y_{r}$. Among these two terms, the first one needs to be expanded further as

$$
\begin{aligned}
T_{2,2}[1]= & \frac{\left(k_{1} \cdot f_{p} \cdot f_{q} \cdot k_{1}\right)}{\mathcal{K}_{1 p}} \frac{\left(k_{1} \cdot f_{r} \cdot\left(Y_{r}+k_{q}+k_{p}\right)\right)}{\mathcal{K}_{1 r}} A_{n+3}^{\mathrm{YM}}(1, q, 2,\{3, \ldots, n-1\} w\{p, r\}, n) \\
& +\frac{\left(k_{1} \cdot f_{p} \cdot f_{q} \cdot k_{1}\right)}{\mathcal{K}_{1 p}} \frac{\left(k_{1} \cdot f_{r} \cdot\left(Y_{r}+k_{q}+k_{p}\right)\right)}{\mathcal{K}_{1 r}} A_{n+3}^{\mathrm{YM}}(1, q, p, 2,\{3, \ldots, n-1\} ш\{r\}, n) \\
& +\frac{\left(k_{1} \cdot f_{p} \cdot f_{q} \cdot k_{1}\right)}{\mathcal{K}_{1 p}} \frac{\left(k_{1} \cdot f_{r} \cdot\left(k_{1}+k_{q}+k_{p}\right)\right)}{\mathcal{K}_{1 r}} A_{n+3}^{\mathrm{YM}}(1, q, p, r,\{2,3, \ldots, n-1\}, n) .
\end{aligned}
$$

Using (2.10), we can read out the contribution to the coefficient $C(\{p, q, r\})$ from the first term as

$$
C^{T_{2,2}[1,1]}(\{p, q, r\})=-\frac{\left(k_{q} \cdot\left(Y_{q}+k_{p}\right)\right)}{\mathcal{K}_{1 q}} \frac{\left(k_{1} \cdot f_{p} \cdot f_{q} \cdot k_{1}\right)}{\mathcal{K}_{1 p}} \frac{\left(k_{1} \cdot f_{r} \cdot\left(Y_{r}+k_{q}+k_{p}\right)\right)}{\mathcal{K}_{1 r}} .
$$

Using (2.11), we can read out the contribution to the coefficient $C(\{p, q, r\})$ from the second term as

$$
C^{T_{2,2}[1,2]}(\{p, q, r\})=\frac{\left(k_{q} \cdot k_{1}+k_{p} \cdot\left(Y_{p}+k_{q}\right)\right)\left(k_{q} \cdot\left(Y_{q}+k_{p}\right)\right)}{\mathcal{K}_{1 p q} \mathcal{K}_{1 q}} \frac{\left(k_{1} \cdot f_{p} \cdot f_{q} \cdot k_{1}\right)}{\mathcal{K}_{1 p}} \frac{\left(k_{1} \cdot f_{r} \cdot\left(Y_{r}+k_{q}+k_{p}\right)\right)}{\mathcal{K}_{1 r}} .
$$

Using (2.21), we can read out the contribution to the coefficient $C_{3}(\{p, q, r\})$ from the third term as ${ }^{17}$

$$
C^{T_{2,2}[1,3]}(\{p, q, r\})=-\frac{k_{q} \cdot X_{q}}{\mathcal{K}_{1 q}} \frac{\left(k_{p} \cdot\left(Y_{p}-k_{1}\right)\right)}{\mathcal{K}_{1 p q}} \frac{k_{r} \cdot X_{r}}{\mathcal{K}_{1 p q r}} \frac{\left(k_{1} \cdot f_{p} \cdot f_{q} \cdot k_{1}\right)}{\mathcal{K}_{1 p}} \frac{\left(k_{1} \cdot f_{r} \cdot\left(k_{1}+k_{q}+k_{p}\right)\right)}{\mathcal{K}_{1 r}} .
$$

Now we sum up the above three coefficients. The sum of the first two is

$$
C^{T_{2,2}[1,1]+T_{2,2}[1,2]}(\{p, q, r\})=\frac{\left(k_{q} \cdot X_{q}\right)}{\mathcal{K}_{1 q}} \frac{\left(k_{1} \cdot f_{p} \cdot f_{q} \cdot k_{1}\right)}{\mathcal{K}_{1 p}} \frac{\left(k_{1} \cdot f_{r} \cdot X_{r}\right)}{\mathcal{K}_{1 r}} \frac{\left(k_{p} \cdot\left(Y_{p}-k_{1}\right)\right)}{\mathcal{K}_{1 p q}}
$$

Adding up the third one we get

\footnotetext{
${ }^{16}$ This is also the reason we consider the coefficient $C(\{p, q, r\})$ for simplicity. Although other coefficients will be similar, in order to lead to the pattern observed from this coefficient, nontrivial algebraic manipulations will be needed.

${ }^{17}$ Now it is clear why in (5.20) we write $X_{r}$ out explicitly, since when we use (2.21) to get (5.23), another $X_{r}$ appears. The meaning of these two $X_{r}$ 's is different and we must make clear distinction.
} 


$$
\begin{aligned}
C^{T_{2,2}[1]}(\{p, q, r\})= & \frac{\left(k_{1} \cdot f_{p} \cdot f_{q} \cdot k_{1}\right)\left(k_{p} \cdot\left(Y_{p}-k_{1}\right)\right)\left(k_{q} \cdot\left(Y_{q}+k_{p}\right)\right)}{\mathcal{K}_{1 p} \mathcal{K}_{1 q} \mathcal{K}_{1 p q}} \times \frac{\left(k_{1} \cdot f_{r} \cdot\left(Y_{r}+k_{p}+k_{q}\right)\right)}{\mathcal{K}_{1 r}} \\
& -\frac{\left(k_{1} \cdot f_{p} \cdot f_{q} \cdot k_{1}\right)\left(k_{p} \cdot\left(Y_{p}-k_{1}\right)\right)\left(k_{q} \cdot\left(Y_{q}+k_{p}\right)\right)}{\mathcal{K}_{1 p} \mathcal{K}_{1 q} \mathcal{K}_{1 p q}} \times \frac{\left(k_{r} \cdot X_{r}\right)}{\mathcal{K}_{1 p q r}} \frac{\left(k_{1} \cdot f_{r} \cdot\left(k_{1}+k_{q}+k_{p}\right)\right)}{\mathcal{K}_{1 r}} .
\end{aligned}
$$

For the second term of $T_{2,2}$, we can expand to another three terms

$$
\begin{aligned}
T_{2,2}[2]= & \frac{\left(k_{1} \cdot f_{p} \cdot f_{q} \cdot k_{1}\right)}{\mathcal{K}_{1 p}} \frac{\left(k_{1} \cdot f_{r} \cdot\left(Y_{r}+k_{q}\right)\right)}{\mathcal{K}_{1 r}} A_{n+3}^{\mathrm{YM}}(1, q, 2,\{3, \ldots, n-1\} ш\{r, p\}, n) \\
& +\frac{\left(k_{1} \cdot f_{p} \cdot f_{q} \cdot k_{1}\right)}{\mathcal{K}_{1 p}} \frac{\left(k_{1} \cdot f_{r} \cdot\left(k_{1}+k_{q}\right)\right)}{\mathcal{K}_{1 r}} A_{n+3}^{\mathrm{YM}}(1, q, r, 2,\{3, \ldots, n-1\} ш\{p\}, n) \\
& +\frac{\left(k_{1} \cdot f_{p} \cdot f_{q} \cdot k_{1}\right)}{\mathcal{K}_{1 p}} \frac{\left(k_{1} \cdot f_{r} \cdot\left(k_{1}+k_{q}\right)\right)}{\mathcal{K}_{1 r}} A_{n+3}^{\mathrm{YM}}(1, q, r, p,\{2,3, \ldots, n-1\}, n) .
\end{aligned}
$$

The first term does not contribute to the coefficient $C(\{p, q, r\})$. Using (2.11) and (2.21), the contributions from the second and third terms are

$$
\begin{aligned}
C^{T_{2,2}[2]}(\{p, q, r\})= & \frac{\left(k_{1} \cdot f_{p} \cdot f_{q} \cdot k_{1}\right)}{\mathcal{K}_{1 p}} \frac{\left(k_{1} \cdot f_{r} \cdot\left(k_{1}+k_{q}\right)\right)}{\mathcal{K}_{1 r}} \frac{\left(k_{q} \cdot\left(Y_{q}+k_{p}-k_{1}\right)\right)\left(k_{r} \cdot\left(Y_{r}+k_{p}+k_{q}\right)\right)}{\mathcal{K}_{1 q r} \mathcal{K}_{1 q}} \\
& -\frac{\left(k_{1} \cdot f_{p} \cdot f_{q} \cdot k_{1}\right)}{\mathcal{K}_{1 p}} \frac{\left(k_{1} \cdot f_{r} \cdot\left(k_{1}+k_{q}\right)\right)}{\mathcal{K}_{1 r}} \frac{\left(k_{q} \cdot\left(X_{q}-k_{1}\right)\right)}{\mathcal{K}_{1 q}} \frac{\left(k_{r} \cdot X_{r}\right)}{\mathcal{K}_{1 q r}} \frac{\left(k_{p} \cdot\left(Y_{p}-k_{1}\right)-\mathcal{K}_{1 p q r}\right)}{\mathcal{K}_{1 p q r}} \\
= & -\frac{\left(k_{1} \cdot f_{p} \cdot f_{q} \cdot k_{1}\right)}{\mathcal{K}_{1 p}} \frac{\left(k_{1} \cdot f_{r} \cdot\left(k_{1}+k_{q}\right)\right)}{\mathcal{K}_{1 r}} \frac{\left(k_{r} \cdot X_{r}\right)}{\mathcal{K}_{1 q r}} \frac{\left(k_{q} \cdot\left(X_{q}-k_{1}\right)\right)}{\mathcal{K}_{1 q}} \frac{\left(k_{p} \cdot\left(Y_{p}-k_{1}\right)\right)}{\mathcal{K}_{1 p q r}} .
\end{aligned}
$$

Putting these together, we get

$$
\begin{aligned}
C^{T_{2,2}}(\{p, q, r\})= & \frac{\left(k_{1} \cdot f_{p} \cdot f_{q} \cdot k_{1}\right)\left(k_{p} \cdot\left(Y_{p}-k_{1}\right)\right)\left(k_{q} \cdot\left(Y_{q}+k_{p}\right)\right)}{\mathcal{K}_{1 p} \mathcal{K}_{1 q} \mathcal{K}_{1 p q}} \frac{\left(k_{1} \cdot f_{r} \cdot\left(Y_{r}+k_{p}+k_{q}\right)\right)}{\mathcal{K}_{1 r}} \\
& -\frac{\left(k_{1} \cdot f_{p} \cdot f_{q} \cdot k_{1}\right)\left(k_{p} \cdot\left(Y_{p}-k_{1}\right)\right)\left(k_{q} \cdot\left(Y_{q}+k_{p}\right)\right)}{\mathcal{K}_{1 p} \mathcal{K}_{1 q} \mathcal{K}_{1 p q}} \frac{\left(k_{r} \cdot X_{r}\right)}{\mathcal{K}_{1 p q r}} \frac{\left(k_{1} \cdot f_{r} \cdot\left(k_{1}+k_{q}+k_{p}\right)\right)}{\mathcal{K}_{1 r}} \\
& -\frac{\left(k_{1} \cdot f_{p} \cdot f_{q} \cdot k_{1}\right)\left(k_{p} \cdot\left(Y_{p}-k_{1}\right)\right)\left(k_{q} \cdot\left(X_{q}-k_{1}\right)\right)}{\mathcal{K}_{1 p} \mathcal{K}_{1 q}} \frac{\left(k_{1} \cdot f_{r} \cdot\left(k_{1}+k_{q}\right)\right)}{\mathcal{K}_{1 r}} \frac{\left(k_{r} \cdot X_{r}\right)}{\mathcal{K}_{1 q r} \mathcal{K}_{1 p q r}} .
\end{aligned}
$$

The third term: For the third term, we expand further as

$$
\begin{aligned}
T_{3}= & \frac{\left(k_{1} \cdot f_{p} \cdot f_{r} \cdot Y_{r}\right)}{\left(k_{p} \cdot k_{1}\right)} A_{n+2,1}^{\mathrm{EYM}}(1,2,\{3, \ldots, n-1\} ш\{r, p\}, n ; q) \\
& +\frac{\left(k_{1} \cdot f_{p} \cdot f_{r} \cdot k_{1}\right)}{\left(k_{p} \cdot k_{1}\right)} A_{n+2,1}^{\mathrm{EYM}}(1, r,\{2, \ldots, n-1\} \uplus\{p\}, n ; q) .
\end{aligned}
$$

Again, the first term does not contribute to the coefficient $C(\{p, q, r\})$. For the second term, using the result of (5.4) we get

$$
\begin{aligned}
T_{3,2}= & \frac{\left(k_{1} \cdot f_{p} \cdot f_{r} \cdot k_{1}\right)}{\left(k_{p} \cdot k_{1}\right)} \frac{\left(k_{1} \cdot f_{q} \cdot X_{q}\right)}{\mathcal{K}_{1 q}} A_{n+3}^{\mathrm{YM}}(1, r,\{2, \ldots, n-1\} ш\{p\} ш\{q\}, n) \\
= & \frac{\left(k_{1} \cdot f_{p} \cdot f_{r} \cdot k_{1}\right)}{\left(k_{p} \cdot k_{1}\right)} \frac{\left(k_{1} \cdot f_{q} \cdot X_{q}\right)}{\mathcal{K}_{1 q}} A_{n+3}^{\mathrm{YM}}(1, r,\{2, \ldots, n-1\} ш\{p, q\}, n) \\
& +\frac{\left(k_{1} \cdot f_{p} \cdot f_{r} \cdot k_{1}\right)}{\left(k_{p} \cdot k_{1}\right)} \frac{\left(k_{1} \cdot f_{q} \cdot X_{q}\right)}{\mathcal{K}_{1 q}} A_{n+3}^{\mathrm{YM}}(1, r,\{2, \ldots, n-1\} ш\{q, p\}, n) .
\end{aligned}
$$


Now we treat these term by term. For the first term, we expand it further as

$$
\begin{aligned}
T_{3,2}[1]= & \frac{\left(k_{1} \cdot f_{p} \cdot f_{r} \cdot k_{1}\right)}{\left(k_{p} \cdot k_{1}\right)} \frac{\left(k_{1} \cdot f_{q} \cdot\left(Y_{q}+k_{r}+k_{p}\right)\right)}{\mathcal{K}_{1 q}} A_{n+3}^{\mathrm{YM}}(1, r, 2,\{3, \ldots, n-1\} w\{p, q\}, n) \\
& +\frac{\left(k_{1} \cdot f_{p} \cdot f_{r} \cdot k_{1}\right)}{\left(k_{p} \cdot k_{1}\right)} \frac{\left(k_{1} \cdot f_{q} \cdot\left(Y_{q}+k_{r}+k_{p}\right)\right)}{\mathcal{K}_{1 q}} A_{n+3}^{\mathrm{YM}}(1, r, p, 2,\{3, \ldots, n-1\} ш\{q\}, n) \\
& +\frac{\left(k_{1} \cdot f_{p} \cdot f_{r} \cdot k_{1}\right)}{\left(k_{p} \cdot k_{1}\right)} \frac{\left(k_{1} \cdot f_{q} \cdot\left(k_{1}+k_{r}+k_{p}\right)\right)}{\mathcal{K}_{1 q}} A_{n+3}^{\mathrm{YM}}(1, r, p, q,\{2, \ldots, n-1\}, n) .
\end{aligned}
$$

Reading out contributions to the coefficient $C(\{p, q, r\})$ we get

$$
\begin{aligned}
C^{T_{3,2}[1]}(\{p, q, r\})= & \frac{\left(k_{1} \cdot f_{p} \cdot f_{r} \cdot k_{1}\right)}{\left(k_{p} \cdot k_{1}\right)} \frac{\left(k_{1} \cdot f_{q} \cdot\left(Y_{q}+k_{r}+k_{p}\right)\right)}{\mathcal{K}_{1 q}} \times \frac{-\left(k_{r} \cdot X_{r}\right)}{\mathcal{K}_{1 r}} \\
& +\frac{\left(k_{1} \cdot f_{p} \cdot f_{r} \cdot k_{1}\right)}{\left(k_{p} \cdot k_{1}\right)} \frac{\left(k_{1} \cdot f_{q} \cdot\left(Y_{q}+k_{r}+k_{p}\right)\right)}{\mathcal{K}_{1 q}} \times \frac{\left(k_{r} \cdot k_{1}+k_{p} \cdot\left(Y_{p}+k_{r}\right)\right)\left(k_{r} \cdot\left(Y_{r}+k_{p}+k_{q}\right)\right)}{\mathcal{K}_{1 p r} \mathcal{K}_{1 r}} \\
& +\frac{\left(k_{1} \cdot f_{p} \cdot f_{r} \cdot k_{1}\right)}{\left(k_{p} \cdot k_{1}\right)} \frac{\left(k_{1} \cdot f_{q} \cdot\left(k_{1}+k_{r}+k_{p}\right)\right)}{\mathcal{K}_{1 q}} \\
& \times \frac{-\left(k_{r} \cdot\left(Y_{r}+k_{p}+k_{q}\right)\right)\left(k_{q} \cdot\left(Y_{q}+k_{r}+k_{p}\right)\right)\left(k_{p} \cdot\left(Y_{p}-k_{1}\right)\right)}{\mathcal{K}_{1 p q r} \mathcal{K}_{1 p r} \mathcal{K}_{1 r}} \\
= & \frac{\left(k_{1} \cdot f_{p} \cdot f_{r} \cdot k_{1}\right)}{\left(k_{p} \cdot k_{1}\right)} \frac{\left(k_{1} \cdot f_{q} \cdot\left(Y_{q}+k_{r}+k_{p}\right)\right)}{\mathcal{K}_{1 q}} \frac{\left(k_{p} \cdot\left(Y_{p}-k_{1}\right)\right)\left(k_{r} \cdot X_{r}\right)}{\mathcal{K}_{1 p r} \mathcal{K}_{1 r}} \\
& -\frac{\left(k_{1} \cdot f_{p} \cdot f_{r} \cdot k_{1}\right)}{\left(k_{p} \cdot k_{1}\right)} \frac{\left(k_{1} \cdot f_{q} \cdot\left(k_{1}+k_{r}+k_{p}\right)\right)}{\mathcal{K}_{1 q}} \frac{\left(k_{r} \cdot X_{r}\right)\left(k_{q} \cdot\left(Y_{q}+k_{r}+k_{p}\right)\right)\left(k_{p} \cdot\left(Y_{p}-k_{1}\right)\right)}{\mathcal{K}_{1 p q r} \mathcal{K}_{1 p r} \mathcal{K}_{1 r}} .
\end{aligned}
$$

For the second term we expand to

$$
\begin{aligned}
T_{3,2}[2]= & \frac{\left(k_{1} \cdot f_{p} \cdot f_{r} \cdot k_{1}\right)}{\left(k_{p} \cdot k_{1}\right)} \frac{\left(k_{1} \cdot f_{q} \cdot\left(Y_{q}+k_{r}\right)\right)}{\mathcal{K}_{1 q}} A_{n+3}^{\mathrm{YM}}(1, r, 2,\{3, \ldots, n-1\} w\{q, p\}, n) \\
& +\frac{\left(k_{1} \cdot f_{p} \cdot f_{r} \cdot k_{1}\right)}{\left(k_{p} \cdot k_{1}\right)} \frac{\left(k_{1} \cdot f_{q} \cdot\left(k_{1}+k_{r}\right)\right)}{\mathcal{K}_{1 q}} A_{n+3}^{\mathrm{YM}}(1, r, q, 2,\{3, \ldots, n-1\} ш\{p\}, n) \\
& +\frac{\left(k_{1} \cdot f_{p} \cdot f_{r} \cdot k_{1}\right)}{\left(k_{p} \cdot k_{1}\right)} \frac{\left(k_{1} \cdot f_{q} \cdot\left(k_{1}+k_{r}\right)\right)}{\mathcal{K}_{1 q}} A_{n+3}^{\mathrm{YM}}(1, r, q, p,\{2, \ldots, n-1\}, n) .
\end{aligned}
$$

Reading out contributions to the coefficient $C(\{p, q, r\})$ we get (the first term does not contribute)

$$
\begin{aligned}
C^{T_{3,2}[2]}(\{p, q, r\})= & \frac{\left(k_{1} \cdot f_{p} \cdot f_{r} \cdot k_{1}\right)}{\left(k_{p} \cdot k_{1}\right)} \frac{\left(k_{1} \cdot f_{q} \cdot\left(k_{1}+k_{r}\right)\right)}{\mathcal{K}_{1 q}} \times \frac{\left(k_{r} \cdot k_{1}+k_{q} \cdot\left(Y_{q}+k_{p}+k_{r}\right)\right)\left(k_{r} \cdot\left(Y_{r}+k_{q}+k_{p}\right)\right)}{\mathcal{K}_{1 q r} \mathcal{K}_{1 r}} \\
& +\frac{\left(k_{1} \cdot f_{p} \cdot f_{r} \cdot k_{1}\right)}{\left(k_{p} \cdot k_{1}\right)} \frac{\left(k_{1} \cdot f_{q} \cdot\left(k_{1}+k_{r}\right)\right)}{\mathcal{K}_{1 q}} \times \frac{-k_{r} \cdot X_{r}}{\mathcal{K}_{1 r}} \times \frac{-k_{q} \cdot\left(X_{q}-k_{1}\right)-\mathcal{K}_{1 q r}}{\mathcal{K}_{1 q r}} \times \frac{-k_{p} \cdot\left(Y_{p}-k_{1}\right)-\mathcal{K}_{1 p q r}}{\mathcal{K}_{1 p q r}} \\
= & -\frac{\left(k_{1} \cdot f_{p} \cdot f_{r} \cdot k_{1}\right)}{\left(k_{p} \cdot k_{1}\right)} \frac{\left(k_{1} \cdot f_{q} \cdot\left(k_{1}+k_{r}\right)\right)}{\mathcal{K}_{1 q}} \frac{\left(k_{r} \cdot X_{r}\right)}{\mathcal{K}_{1 r}} \frac{\left(k_{q} \cdot\left(X_{q}-k_{1}\right)+\mathcal{K}_{1 q r}\right)}{\mathcal{K}_{1 q r}} \frac{\left(k_{p} \cdot\left(Y_{p}-k_{1}\right)\right)}{\mathcal{K}_{1 p q r}}
\end{aligned}
$$

Putting two parts together we get 


$$
\begin{aligned}
C^{T_{3,2}}(\{p, q, r\})= & \frac{\left(k_{1} \cdot f_{p} \cdot f_{r} \cdot k_{1}\right)}{\left(k_{p} \cdot k_{1}\right)} \frac{\left(k_{1} \cdot f_{q} \cdot\left(Y_{q}+k_{r}+k_{p}\right)\right)}{\mathcal{K}_{1 q}} \frac{\left(k_{p} \cdot\left(Y_{p}-k_{1}\right)\right)\left(k_{r} \cdot X_{r}\right)}{\mathcal{K}_{1 p r} \mathcal{K}_{1 r}} \\
& -\frac{\left(k_{1} \cdot f_{p} \cdot f_{r} \cdot k_{1}\right)}{\left(k_{p} \cdot k_{1}\right)} \frac{\left(k_{1} \cdot f_{q} \cdot\left(k_{1}+k_{r}+k_{p}\right)\right)}{\mathcal{K}_{1 q}} \frac{\left(k_{r} \cdot X_{r}\right)\left(k_{q} \cdot\left(Y_{q}+k_{r}+k_{p}\right)\right)\left(k_{p} \cdot\left(Y_{p}-k_{1}\right)\right)}{\mathcal{K}_{1 p q r} \mathcal{K}_{1 p r} \mathcal{K}_{1 r}} \\
& -\frac{\left(k_{1} \cdot f_{p} \cdot f_{r} \cdot k_{1}\right)}{\left(k_{p} \cdot k_{1}\right)} \frac{\left(k_{1} \cdot f_{q} \cdot\left(k_{1}+k_{r}\right)\right)}{\mathcal{K}_{1 q}} \frac{\left(k_{r} \cdot X_{r}\right)}{\mathcal{K}_{1 r}} \frac{\left(k_{q} \cdot\left(X_{q}-k_{1}\right)+\mathcal{K}_{1 q r}\right)}{\mathcal{K}_{1 q r}} \frac{\left(k_{p} \cdot\left(Y_{p}-k_{1}\right)\right)}{\mathcal{K}_{1 p q r}} .
\end{aligned}
$$

The fourth term: The fourth term can be expanded to four terms:

$$
\begin{aligned}
T_{4}= & \frac{\left(k_{1} \cdot f_{p} \cdot f_{q} \cdot f_{r} \cdot Y_{r}\right)}{\left(k_{p} \cdot k_{1}\right)} A_{n+3}^{\mathrm{YM}}(1,2,\{3, \ldots, n-1\} ш\{r, q, p\}, n) \\
& +\frac{\left(k_{1} \cdot f_{p} \cdot f_{q} \cdot f_{r} \cdot k_{1}\right)}{\left(k_{p} \cdot k_{1}\right)} A_{n+3}^{\mathrm{YM}}(1, r, 2,\{3, \ldots, n-1\} ш\{q, p\}, n) \\
& +\frac{\left(k_{1} \cdot f_{p} \cdot f_{q} \cdot f_{r} \cdot k_{1}\right)}{\left(k_{p} \cdot k_{1}\right)} A_{n+3}^{\mathrm{YM}}(1, r, q, 2,\{3, \ldots, n-1\} ш\{p\}, n) \\
& +\frac{\left(k_{1} \cdot f_{p} \cdot f_{q} \cdot f_{r} \cdot k_{1}\right)}{\left(k_{p} \cdot k_{1}\right)} A_{n+3}^{\mathrm{YM}}(1, r, q, p,\{2, \ldots, n-1\}, n) .
\end{aligned}
$$

The first and the second terms do not contribute to the coefficient $C(\{p, q, r\})$. The remaining two terms give

$$
\begin{aligned}
C^{T_{4}}(\{p, q, r\})= & \frac{\left(k_{1} \cdot f_{p} \cdot f_{q} \cdot f_{r} \cdot k_{1}\right)}{\left(k_{p} \cdot k_{1}\right)} \times \frac{\left(k_{r} \cdot k_{1}+k_{q} \cdot\left(Y_{q}+k_{r}+k_{p}\right)\right)\left(k_{r} \cdot\left(Y_{r}+k_{q}+k_{p}\right)\right)}{\mathcal{K}_{1 q r} \mathcal{K}_{1 r}} \\
& +\frac{\left(k_{1} \cdot f_{p} \cdot f_{q} \cdot f_{r} \cdot k_{1}\right)}{\left(k_{p} \cdot k_{1}\right)} \times \frac{-k_{r} \cdot X_{r}}{\mathcal{K}_{1 r}} \times \frac{-k_{q} \cdot\left(X_{q}-k_{1}\right)-\mathcal{K}_{1 q r}}{\mathcal{K}_{1 q r}} \times \frac{-k_{p} \cdot\left(Y_{p}-k_{1}\right)-\mathcal{K}_{1 p q r}}{\mathcal{K}_{1 p q r}} \\
= & -\frac{\left(k_{1} \cdot f_{p} \cdot f_{q} \cdot f_{r} \cdot k_{1}\right)}{\left(k_{p} \cdot k_{1}\right)} \frac{\left(k_{r} \cdot X_{r}\right)}{\mathcal{K}_{1 r}} \frac{\left(k_{q} \cdot\left(X_{q}-k_{1}\right)+\mathcal{K}_{1 q r}\right)}{\mathcal{K}_{1 q r}} \frac{\left(k_{p} \cdot\left(Y_{p}-k_{1}\right)\right)}{\mathcal{K}_{1 p q r}} .
\end{aligned}
$$

The fifth term: We expand it to four terms

$$
\begin{aligned}
T_{5}= & \frac{\left(k_{1} \cdot f_{p} \cdot f_{r} \cdot f_{q} \cdot Y_{q}\right)}{\left(k_{p} \cdot k_{1}\right)} A_{n+3}^{\mathrm{YM}}(1,2,\{3, \ldots, n-1\} ш\{q, r, p\}, n) \\
& +\frac{\left(k_{1} \cdot f_{p} \cdot f_{r} \cdot f_{q} \cdot k_{1}\right)}{\left(k_{p} \cdot k_{1}\right)} A_{n+3}^{\mathrm{YM}}(1, q, 2,\{3, \ldots, n-1\} ш\{r, p\}, n) \\
& +\frac{\left(k_{1} \cdot f_{p} \cdot f_{r} \cdot f_{q} \cdot k_{1}\right)}{\left(k_{p} \cdot k_{1}\right)} A_{n+3}^{\mathrm{YM}}(1, q, r, 2,\{3, \ldots, n-1\} ш\{p\}, n) \\
& +\frac{\left(k_{1} \cdot f_{p} \cdot f_{r} \cdot f_{q} \cdot k_{1}\right)}{\left(k_{p} \cdot k_{1}\right)} A_{n+3}^{\mathrm{YM}}(1, q, r, p,\{2, \ldots, n-1\}, n) .
\end{aligned}
$$

Again, the first and the second terms do not contribute to coefficient $C(\{p, q, r\})$. The remaining two terms give

$$
\begin{aligned}
C^{T_{5}}(\{p, q, r\})= & \frac{\left(k_{1} \cdot f_{p} \cdot f_{r} \cdot f_{q} \cdot k_{1}\right)}{\left(k_{p} \cdot k_{1}\right)} \times \frac{\left(k_{q} \cdot\left(Y_{q}+k_{p}-k_{1}\right)\right)\left(k_{r} \cdot\left(Y_{r}+k_{q}+k_{p}\right)\right)}{\mathcal{K}_{1 q r} \mathcal{K}_{1 q}} \\
& +\frac{\left(k_{1} \cdot f_{p} \cdot f_{r} \cdot f_{q} \cdot k_{1}\right)}{\left(k_{p} \cdot k_{1}\right)} \times \frac{-\left(k_{q} \cdot\left(X_{q}-k_{1}\right)\right)}{\mathcal{K}_{1 q}} \times \frac{-k_{r} \cdot X_{r}}{\mathcal{K}_{1 q r}} \times \frac{-k_{p} \cdot\left(Y_{p}-k_{1}\right)-\mathcal{K}_{1 p q r}}{\mathcal{K}_{1 p q r}} \\
= & -\frac{\left(k_{1} \cdot f_{p} \cdot f_{r} \cdot f_{q} \cdot k_{1}\right)}{\left(k_{p} \cdot k_{1}\right)} \frac{\left(k_{q} \cdot\left(X_{q}-k_{1}\right)\right)}{\mathcal{K}_{1 q}} \frac{\left(k_{r} \cdot X_{r}\right)}{\mathcal{K}_{1 q r}} \frac{\left(k_{p} \cdot\left(Y_{p}-k_{1}\right)\right)}{\mathcal{K}_{1 p q r}}
\end{aligned}
$$


In total: Now we sum up these contributions and simplify them to

$$
\begin{aligned}
& \frac{\left(k_{1} \cdot f_{p} \cdot X_{p}\right)}{\mathcal{K}_{1 p}} \frac{\left(k_{1} \cdot f_{q} \cdot X_{q}\right)}{\mathcal{K}_{1 q}} \frac{\left(k_{1} \cdot f_{r} \cdot X_{r}\right)}{\mathcal{K}_{1 r}}+\frac{\left(k_{1} \cdot f_{p} \cdot X_{p}\right)}{\left(k_{p} \cdot k_{1}\right)} \frac{\left(k_{1} \cdot f_{q} \cdot f_{r} \cdot k_{1}\right)\left(k_{q} \cdot\left(X_{q}-k_{1}\right)\right)\left(k_{r} \cdot X_{r}\right)}{\mathcal{K}_{1 q r} \mathcal{K}_{1 q} \mathcal{K}_{1 r}} \\
& +\frac{\left(k_{1} \cdot f_{p} \cdot f_{q} \cdot k_{1}\right)\left(k_{p} \cdot\left(X_{p}-k_{1}\right)\right)\left(k_{q} \cdot X_{q}\right)}{\mathcal{K}_{1 p} \mathcal{K}_{1 q} \mathcal{K}_{1 p q}} \frac{\left(k_{1} \cdot f_{r} \cdot X_{r}\right)}{\mathcal{K}_{1 r}}+\frac{\left(k_{1} \cdot f_{p} \cdot f_{r} \cdot k_{1}\right)\left(k_{p} \cdot\left(X_{p}-k_{1}\right)\right)\left(k_{r} \cdot X_{r}\right)}{\mathcal{K}_{1 p} \mathcal{K}_{1 p r} \mathcal{K}_{1 r}} \frac{\left(k_{1} \cdot f_{q} \cdot X_{q}\right)}{\mathcal{K}_{1 q}} \\
& \quad-\frac{\left(k_{p} \cdot\left(X_{p}-k_{1}\right)\right)\left(k_{r} \cdot X_{r}\right)}{\mathcal{K}_{1 p} \mathcal{K}_{1 q} \mathcal{K}_{1 r} \mathcal{K}_{1 p q r}}\left\{\frac{\left(k_{1} \cdot f_{p} \cdot f_{q} \cdot k_{1}\right)\left(k_{1} \cdot f_{r} \cdot k_{p}\right)\left(k_{q} \cdot X_{q}\right)}{\mathcal{K}_{1 p q}}+\frac{\left(k_{1} \cdot f_{p} \cdot f_{r} \cdot k_{1}\right)\left(k_{1} \cdot f_{q} \cdot k_{p}\right)\left(k_{q} \cdot\left(X_{q}+k_{r}\right)\right)}{\mathcal{K}_{1 p r}}\right. \\
& \quad+\left(k_{1} \cdot f_{p} \cdot f_{q} \cdot k_{1}\right)\left(k_{1} \cdot f_{r} \cdot k_{q}\right)\left\{\frac{\left(k_{q} \cdot X_{q}\right)}{\mathcal{K}_{1 p q}}+\frac{\left(k_{q} \cdot\left(X_{q}-k_{1}\right)\right)}{\mathcal{K}_{1 q r}}\right\}+\frac{\left(k_{1} \cdot f_{p} \cdot f_{q} \cdot f_{r} \cdot k_{1}\right)\left(k_{1} \cdot k_{q}\right)\left(k_{q} \cdot\left(X_{q}-k_{1}\right)+\mathcal{K}_{1 q r}\right)}{\mathcal{K}_{1 q r}} \\
& \left.\quad+\left(k_{1} \cdot f_{p} \cdot f_{r} \cdot k_{1}\right)\left(k_{1} \cdot f_{q} \cdot k_{r}\right)\left\{\frac{\left(k_{q} \cdot\left(Y_{q}-k_{1}\right)\right)}{\mathcal{K}_{1 p r}}+\frac{\left(k_{q} \cdot\left(X_{q}-k_{1}\right)\right)}{\mathcal{K}_{1 q r}}\right\}+\frac{\left(k_{1} \cdot f_{p} \cdot f_{r} \cdot f_{q} \cdot k_{1}\right)\left(k_{1} \cdot k_{r}\right)\left(k_{q} \cdot\left(X_{q}-k_{1}\right)\right)}{\mathcal{K}_{1 q r}}\right\}
\end{aligned}
$$

We can simplify further, by using

$$
\begin{aligned}
\left(k_{1} \cdot f_{p} \cdot f_{q} \cdot f_{r} \cdot k_{1}\right)\left(k_{1} \cdot k_{q}\right) & =\left(k_{1} \cdot f_{p} \cdot f_{q} \cdot k_{1}\right)\left(k_{q} \cdot f_{r} \cdot k_{1}\right)+\left(k_{1} \cdot f_{p} \cdot k_{q}\right)\left(k_{1} \cdot f_{q} \cdot f_{r} \cdot k_{1}\right) \\
\left(k_{1} \cdot f_{p} \cdot f_{r} \cdot f_{q} \cdot k_{1}\right)\left(k_{1} \cdot k_{r}\right) & =\left(k_{1} \cdot f_{p} \cdot f_{r} \cdot k_{1}\right)\left(k_{r} \cdot f_{q} \cdot k_{1}\right)+\left(k_{1} \cdot f_{p} \cdot k_{r}\right)\left(k_{1} \cdot f_{r} \cdot f_{q} \cdot k_{1}\right)
\end{aligned}
$$

to get

$$
C(\{p, q, r\})=A_{1}+A_{2}+A_{3}
$$

where

$$
\begin{gathered}
A_{1}=\frac{\left(k_{1} \cdot f_{p} \cdot X_{p}\right)}{\mathcal{K}_{1 p}} \frac{\left(k_{1} \cdot f_{q} \cdot X_{q}\right)}{\mathcal{K}_{1 q}} \frac{\left(k_{1} \cdot f_{r} \cdot X_{r}\right)}{\mathcal{K}_{1 r}} \\
A_{2}=\frac{\left(k_{1} \cdot f_{p} \cdot X_{p}\right)}{\left(k_{p} \cdot k_{1}\right)} \times \frac{\left(k_{1} \cdot f_{q} \cdot f_{r} \cdot k_{1}\right)\left(k_{q} \cdot\left(X_{q}-k_{1}\right)\right)\left(k_{r} \cdot X_{r}\right)}{\mathcal{K}_{1 q r} \mathcal{K}_{1 q} \mathcal{K}_{1 r}}+\frac{\left(k_{1} \cdot f_{p} \cdot f_{q} \cdot k_{1}\right)\left(k_{p} \cdot\left(X_{p}-k_{1}\right)\right)\left(k_{q} \cdot X_{q}\right)}{\mathcal{K}_{1 p} \mathcal{K}_{1 q} \mathcal{K}_{1 p q}} \frac{\left(k_{1} \cdot f_{r} \cdot X_{r}\right)}{\mathcal{K}_{1 r}} \\
+\frac{\left(k_{1} \cdot f_{p} \cdot f_{r} \cdot k_{1}\right)\left(k_{p} \cdot\left(X_{p}-k_{1}\right)\right)\left(k_{r} \cdot X_{r}\right)}{\mathcal{K}_{1 p} \mathcal{K}_{1 p r} \mathcal{K}_{1 r}} \frac{\left(k_{1} \cdot f_{q} \cdot X_{q}\right)}{\mathcal{K}_{1 q}} \\
A_{3}=-\frac{\left(k_{p} \cdot\left(X_{p}-k_{1}\right)\right)\left(k_{r} \cdot X_{r}\right)}{\mathcal{K}_{1 p} \mathcal{K}_{1 q} \mathcal{K}_{1 r} \mathcal{K}_{1 p q r}} \times\left\{\left(k_{1} \cdot f_{p} \cdot f_{q} \cdot k_{1}\right)\left(k_{1} \cdot f_{r} \cdot k_{p}\right) \frac{\left(k_{q} \cdot X_{q}\right)}{\mathcal{K}_{1 p q}}+\left(k_{1} \cdot f_{p} \cdot f_{r} \cdot k_{1}\right)\left(k_{1} \cdot f_{q} \cdot k_{p}\right) \frac{\left(k_{q} \cdot\left(X_{q}+k_{r}\right)\right)}{\mathcal{K}_{1 p r}}\right. \\
+\left(k_{1} \cdot f_{p} \cdot f_{q} \cdot k_{1}\right)\left(k_{1} \cdot f_{r} \cdot k_{q}\right) \frac{\left(\left(k_{q} \cdot X_{q}\right)-\mathcal{K}_{1 p q}\right)}{\mathcal{K}_{1 p q}}+\left(k_{1} \cdot f_{q} \cdot f_{r} \cdot k_{1}\right)\left(k_{1} \cdot f_{p} \cdot k_{q}\right) \frac{\left(k_{q} \cdot\left(X_{q}-k_{1}\right)+\mathcal{K}_{1 q r}\right)}{\mathcal{K}_{1 q r}} \\
\left.+\left(k_{1} \cdot f_{p} \cdot f_{r} \cdot k_{1}\right)\left(k_{1} \cdot f_{q} \cdot k_{r}\right) \frac{\left(k_{q} \cdot\left(Y_{q}-k_{1}\right)\right)}{\mathcal{K}_{1 p r}}+\left(k_{1} \cdot f_{q} \cdot f_{r} \cdot k_{1}\right)\left(k_{1} \cdot f_{p} \cdot k_{r}\right) \frac{\left(k_{q} \cdot\left(X_{q}-k_{1}\right)\right)}{\mathcal{K}_{1 q r}}\right\}
\end{gathered}
$$

This is a much nicer expression than the one given by our direct calculation (5.39), since each $A_{i}$ has more manifest physical pattern.

\section{EXPANSION IN THE BCJ BASIS BY DIFFERENTIAL OPERATORS}

In the previous section, we have illustrated how to expand SEYM amplitudes in the BCJ basis of YM amplitudes starting from their expansion in the KK basis. Although the whole procedure is systematical, the algebraic manipulation is not so easy. As we have emphasized, the strategy of using differential operators is itself an independent method; thus, in this section, we will show how to do the calculation. 
Before going to the calculation details, let us give some general considerations. A sEYM amplitude $A_{n, m}^{\mathrm{EYM}}(1,2$, $\left.\ldots, n ;\left\{h_{1}, h_{2}, \ldots, h_{m}\right\}\right)$ with $n \geq 3$ gluons $^{18}$ and $m$ gravitons can be expanded in the BCJ basis of YM amplitudes as follows:

$$
\begin{aligned}
A_{n, m}^{\mathrm{EYM}} & \left(1,2, \ldots, n ;\left\{h_{1}, h_{2}, \ldots, h_{m}\right\}\right) \\
= & \sum_{ш} \sum_{\rho} C(ш, \rho) A_{n+m}^{\mathrm{YM}}(1,2,\{3, \ldots, n-1\} \\
& \left.\times \amalg\left\{\rho_{1}, \ldots, \rho_{m}\right\}, n\right),
\end{aligned}
$$

where the color order of $n$ gluons is kept and $\rho$ represents a permutation of $m$ gravitons, $\rho\left\{h_{1}, h_{2}, \ldots, h_{m}\right\}=$ $\left\{\rho_{1}, \rho_{2}, \ldots, \rho_{m}\right\}$. The coefficients $C(\omega, \rho)$ depend on the permutation $\rho$ and the shuffle $\omega$, because gravitons do not have color order and this can occur in all possible orderings.

By the double copy structure (1.3), only the $\epsilon$ part of graviton polarization tensors $\epsilon^{\mu \nu}=\epsilon^{\mu} \tilde{\epsilon}^{\nu}$ appears in the coefficients $C$, i.e., they are functions of momenta $k_{i}$ 's of all particles and polarization vectors $\epsilon_{i}$ of all gravitons,

$C(\omega, \rho)=C_{ш, \rho}\left(k_{1}, \ldots, k_{n}, k_{h_{1}}, \ldots, k_{h_{m}}, \epsilon_{h_{1}}, \ldots, \epsilon_{h_{m}}\right)$.

A very important fact of the expansion (6.1) is that by the gauge invariance of EYM amplitudes, when $\epsilon_{i}$ is replaced by $k_{i}, A_{n, m}^{\mathrm{EYM}}\left(\epsilon_{i} \rightarrow k_{i}\right)=0$ at the left-hand side, thus $C_{ш, \rho}\left(\epsilon_{i} \rightarrow k_{i}\right)$ must be zero at the right-hand side because each amplitude of the BCJ basis is independent to each other. In other words, these coefficients are also gauge-invariant-like amplitudes. From experiences in the previous section we see that these coefficients are functions of field strength $f^{\mu \nu}$ only and thus are manifestly gauge invariant.

Another general feature is that coefficients are multilinear functions of polarization vectors $\epsilon_{i}$. Thus, Lorentz invariance means coefficients can only be polynomial functions of Lorentz invariant contractions $\left(\epsilon_{i} \cdot \epsilon_{j}\right)$ 's and $\left(\epsilon_{i} \cdot k_{j}\right)$ 's, but can be rational functions of $\left(k_{i} \cdot k_{j}\right)$ 's. We can separate terms according to contraction types sketchily as

$$
\begin{aligned}
C(\amalg, \rho)= & \alpha_{0}(\epsilon \cdot k)^{m}+\alpha_{1}(\epsilon \cdot \epsilon)(\epsilon \cdot k)^{m-2} \\
& +\alpha_{2}(\epsilon \cdot \epsilon)^{2}(\epsilon \cdot k)^{m-4}+\cdots \\
& +\alpha_{\left[\frac{m}{2}\right]}(\epsilon \cdot \epsilon)^{\left[\frac{m}{2}\right]}(\epsilon \cdot k)^{m-2\left[\frac{m}{2}\right]}
\end{aligned}
$$

Above, two general considerations are very important since if we impose such conditions, i.e., the gauge

\footnotetext{
${ }^{18}$ The case $n=2$ is special and we will not discuss it in this paper.
}

invariance and multilinearity of polarization vectors $\epsilon_{i}$, we could derive the expansion of sEYM amplitudes in the BCJ basis naturally as will be shown in this section. Comparing to expansion in the KK basis, building blocks used in this section are much more complicated. In Appendix B, we will give a more systematical discussion about the building blocks for the expansion in the BCJ basis, although complete understanding is still not clear for us.

Now we present several examples to show how to derive the expansion of sEYM amplitudes in the BCJ basis by differential operators.

\section{A. The case of one graviton}

Using the knowledge of building blocks for one polarization vector [see (B6) in Appendix B], we can expand the sEYM amplitude with one graviton as

$$
A_{n, 1}^{\mathrm{EYM}}(1,2, \ldots, n ; p)=\sum_{a=2}^{n-1} \frac{\left(k_{1} f_{p} K_{a}\right)}{\left(k_{1} k_{p}\right)} B_{a} .
$$

Applying $\mathcal{T}_{j p(j+1)}$ (with $2 \leq j \leq n-1$ ) on the above expansion (6.4), the left-hand side gives

$$
\begin{aligned}
\mathcal{L} & =\mathcal{T}_{j p(j+1)} A_{n, 1}^{\mathrm{EYM}}(1,2, \ldots, n ; p) \\
& =A_{n+1}^{\mathrm{YM}}(1,2, \ldots, j, p, j+1, \ldots, n),
\end{aligned}
$$

while the right-hand side gives

$$
\mathcal{R}=\sum_{a=2}^{n-1}\left\{\mathcal{T}_{j p(j+1)} \frac{\left(k_{1} f_{p} K_{a}\right)}{\left(k_{1} k_{p}\right)}\right\} B_{a}=\sum_{i=2}^{n-1} \delta_{j a} B_{a}=B_{j} .
$$

Comparing two sides, we solve

$$
B_{j}=A_{n+1}^{\mathrm{YM}}(1,2, \ldots, j, p, j+1, \ldots, n) .
$$

Putting it back, we get the wanted expansion in the BCJ basis

$$
\begin{aligned}
& A_{n, 1}^{\mathrm{EYM}}(1,2, \ldots, n ; p) \\
& \quad=\sum_{ш} \frac{\left(k_{1} f_{p} Y_{p}\right)}{\left(k_{1} k_{p}\right)} A_{n+1}^{\mathrm{YM}}(1,2,\{3, \ldots, n-1\} ш\{p\}, n),
\end{aligned}
$$

where $K_{a}$ is exactly the $Y_{p}$ defined before. $Y_{p}$ has implicit dependence on the shuffle. We want to emphasize that using insertion operators and gauge invariance for the building blocks only, we have found the expansion of sEYM amplitudes with single graviton in the BCJ basis of YM amplitudes without any other preassumption, such as the KLT relations. 
An interesting observation is that among $(n-1)$ independent insertion operators, we have used only $(n-2)$ of them. Now let us consider the action of $\mathcal{T}_{j p(j+1)}$ with $j=1$ on (6.8):

$$
\begin{aligned}
\mathcal{L} & =\mathcal{T}_{1 p 2} A_{n, 1}^{\mathrm{EYM}}(1,2, \ldots, n ; p)=A_{n+1}^{\mathrm{YM}}(1, p, 2, \ldots, n) \\
\mathcal{R} & =\sum_{a=2}^{n-1}\left\{\mathcal{T}_{1 p 2} \frac{\left(k_{1} f_{p} K_{a}\right)}{\left(k_{1} k_{p}\right)}\right\} A_{n+1}^{\mathrm{YM}}(1,2, \ldots, a, p, a+1, \ldots, n-1, n) \\
& =\frac{1}{\left(k_{p} \cdot k_{1}\right)} \sum_{a=2}^{n-1}\left\{\sum_{t=2}^{a}\left(-k_{1} \cdot k_{p}\right) \delta_{t 2}-\left(k_{p} \cdot k_{t}\right)\right\} A_{n+1}^{\mathrm{YM}}(1,2, \ldots, a, p, a+1, \ldots, n-1, n) \\
& =\sum_{a=2}^{n-1}\left(-k_{p} \cdot K_{a}\right) A_{n+1}^{\mathrm{YM}}(1,2, \ldots, a, p, a+1, \ldots, n-1, n) .
\end{aligned}
$$

Identifying both sides and simplifying, we get

$$
\sum_{ш}\left(k_{p} \cdot X_{p}\right) A_{n+1}^{\mathrm{YM}}(1,\{2, \ldots, n-1\} ш\{p\}, n)=0,
$$

which is nothing, but the fundamental BCJ relation (2.9). The derivation of such an important relation using differential operators shows the deep connection between BCJ relations and the gauge invariance. Result (6.9) can also be understood from another angle: when identifying $\mathcal{L}=\mathcal{R}$, we get the expansion of the left-hand side in the BCJ basis as reviewed in (2.13).

\section{B. The case with two gravitons}

Using the results given in (B13) in Appendix B, we can expand the sEYM amplitude with two gravitons according to the building blocks

$$
\begin{aligned}
A_{n, 2}^{\mathrm{EYM}}(1,2, \ldots, n ; p, q)= & \sum_{a=2}^{n-1} \sum_{b=2}^{n-1} \frac{\left(k_{1} f_{p} K_{a}\right)}{\left(k_{1} k_{p}\right)} \frac{\left(k_{1} f_{q} K_{b}\right)}{\left(k_{1} k_{q}\right)} B_{a b}+\sum_{b=2}^{n-1} \frac{\left(k_{1} \cdot f_{p} \cdot k_{q}\right)}{\left(k_{1} \cdot k_{p}\right)} \frac{\left(k_{1} f_{q} K_{b}\right)}{\left(k_{1} k_{q}\right)} B_{q b} \\
& +\sum_{a=2}^{n-1} \frac{\left(k_{1} \cdot f_{q} \cdot k_{p}\right)}{\left(k_{1} \cdot k_{q}\right)} \frac{\left(k_{1} f_{p} K_{a}\right)}{\left(k_{1} k_{p}\right)} B_{a p}+\frac{\left(k_{1} \cdot f_{p} \cdot f_{q} \cdot k_{1}\right)}{\left(k_{1} \cdot k_{p}\right)\left(k_{1} \cdot k_{q}\right)} D+\frac{\left(k_{1} \cdot f_{p} \cdot k_{q}\right)}{\left(k_{1} \cdot k_{p}\right)} \frac{\left(k_{1} \cdot f_{q} \cdot k_{p}\right)}{\left(k_{1} \cdot k_{q}\right)} E .
\end{aligned}
$$

Now we determine these coefficients one by one:

(i) For E: Since the building block contains the index circle structure $\left(k_{1} \cdot f_{q} \cdot f_{p} \cdot k_{q}\right)$, i.e.,

$$
\left(k_{1} \cdot f_{p} \cdot k_{q}\right)\left(k_{1} \cdot f_{q} \cdot k_{p}\right)=\left(k_{1} \cdot f_{q} \cdot f_{p} \cdot k_{q}\right)\left(k_{1} \cdot k_{p}\right)-\left(k_{1} \cdot f_{p} \cdot f_{q} \cdot k_{1}\right)\left(k_{q} \cdot k_{p}\right)
$$

according to the general argument given in Appendix A, $E=0$.

(ii) For $B_{a b}$ : For this one, we consider the actions of $\mathcal{T}_{j p(j+1)} \mathcal{T}_{m q(m+1)}$ with $j, m=2, \ldots, n-1$. These operators will annihilate the later three terms in (6.11). Using the result

$$
\mathcal{T}_{j p(j+1)} \frac{\left(k_{1} f_{p} K_{a}\right)}{\left(k_{1} k_{p}\right)}= \begin{cases}\delta_{j a}, & j, a \geq 2 \\ -\frac{k_{p} \cdot K_{a}}{k_{p} \cdot k_{1}}, & j=1, a \geq 2\end{cases}
$$

we can solve

$$
B_{a b}= \begin{cases}A_{n+2}^{\mathrm{YM}}(1,2, \ldots, a, p, a+1, \ldots, b, q, b+1, \ldots, n), & a<b \\ A_{n+2}^{\mathrm{YM}}(1,2, \ldots, b, q, b+1, \ldots, a, p, a+1, \ldots, n), & a>b . \\ A_{n+2}^{\mathrm{YM}}(1,2, \ldots, a, p, q, a+1, \ldots, n)+A_{n+2}^{\mathrm{YM}}(1,2, \ldots, a, q, p, a+1, \ldots, n), & a=b\end{cases}
$$


Putting them back, (6.11) becomes

$$
\begin{aligned}
A_{n, 2}^{\mathrm{EYM}}(1,2, \ldots, n ; p, q)= & \sum_{\varpi} \frac{\left(k_{1} f_{p} Y_{p}\right)}{\left(k_{1} k_{p}\right)} \frac{\left(k_{1} f_{q} Y_{q}\right)}{\left(k_{1} k_{q}\right)} A_{n+2}^{\mathrm{YM}}(1,2,\{3, \ldots, n-1\} w\{p\} w\{q\}, n) \\
& +\sum_{b=2}^{n-1} \frac{\left(k_{1} \cdot f_{p} \cdot k_{q}\right)}{\left(k_{1} \cdot k_{p}\right)} \frac{\left(k_{1} f_{q} K_{b}\right)}{\left(k_{1} k_{q}\right)} B_{q b}+\sum_{a=2}^{n-1} \frac{\left(k_{1} \cdot f_{q} \cdot k_{p}\right)}{\left(k_{1} \cdot k_{q}\right)} \frac{\left(k_{1} f_{p} K_{a}\right)}{\left(k_{1} k_{p}\right)} B_{a p}+\frac{\left(k_{1} \cdot f_{p} \cdot f_{q} \cdot k_{1}\right)}{\left(k_{1} \cdot k_{p}\right)\left(k_{1} \cdot k_{q}\right)} D .
\end{aligned}
$$

(iii) For $B_{q b}$ : For this one, let us apply operators $\mathcal{T}_{m q(m+1)}$ with $m=2, \ldots,(n-1)$ first. It will annihilate the third and fourth terms in (6.15) and we get the middle result

$$
\begin{aligned}
& A_{n+1,1}^{\mathrm{EYM}}(1,2, \ldots, m, q, m+1, \ldots, n ; p) \\
& \quad=\sum_{\Psi} \frac{\left(k_{1} f_{p} Y_{p}\right)}{\left(k_{1} k_{p}\right)} A_{n+2}^{\mathrm{YM}}(1,2,\{3, \ldots, m, q, m+1, \ldots, n-1\} ш\{p\}, n)+\frac{\left(k_{1} \cdot f_{p} \cdot k_{q}\right)}{\left(k_{1} \cdot k_{p}\right)} B_{q m} .
\end{aligned}
$$

Next, we apply $\mathcal{T}_{m p q}$ and reach

$$
A_{n+2}^{\mathrm{YM}}(1,2, \ldots, m, p, q, m+1, \ldots, n)=\sum_{ш} A_{n+2}^{\mathrm{YM}}(1,2, \ldots, m,\{q, m+1, \ldots, n-1\} ш\{p\}, n)-B_{q m} .
$$

Thus, we can solve

$$
\begin{aligned}
B_{q m} & =\sum_{\varpi} A_{n+2}^{\mathrm{YM}}(1,2, \ldots, m,\{q, m+1, \ldots, n-1\} ш\{p\}, n)-A_{n+2}^{\mathrm{YM}}(1,2, \ldots, m, p, q, m+1, \ldots, n) \\
& =\sum_{\varpi} A_{n+2}^{\mathrm{YM}}(1,2, \ldots, m, q,\{m+1, \ldots, n-1\} \varpi\{p\}, n) .
\end{aligned}
$$

(iv) For $B_{a p}$ : For this one, let us apply operators $\mathcal{T}_{m p(m+1)}$ with $m=2, \ldots,(n-1)$ first. It will annihilate the second and fourth terms in (6.15) and we get the middle result

$$
\begin{aligned}
& A_{n+1,1}^{\mathrm{EYM}}(1,2, \ldots, m, p, m+1, \ldots, n ; q) \\
& \quad=\sum_{\varpi} \frac{\left(k_{1} f_{q} Y_{q}\right)}{\left(k_{1} k_{q}\right)} A_{n+2}^{\mathrm{YM}}(1,2,\{3, \ldots, m, p, m+1, \ldots, n-1\} ш\{q\}, n)+\frac{\left(k_{1} \cdot f_{q} \cdot k_{p}\right)}{\left(k_{1} \cdot k_{q}\right)} B_{m p} .
\end{aligned}
$$

Next, we apply $\mathcal{T}_{m q p}$ and reach

$$
A_{n+2}^{\mathrm{YM}}(1,2, \ldots, m, q, p, m+1, \ldots, n)=\sum_{山} A_{n+2}^{\mathrm{YM}}(1,2, \ldots, m,\{p, m+1, \ldots, n-1\} ш\{q\}, n)-B_{m p} .
$$

Thus, we can solve

$$
\begin{aligned}
B_{m p} & =\sum_{\varpi} A_{n+2}^{\mathrm{YM}}(1,2, \ldots, m,\{p, m+1, \ldots, n-1\} ш\{q\}, n)-A_{n+2}^{\mathrm{YM}}(1,2, \ldots, m, q, p, m+1, \ldots, n) \\
& =\sum_{\varpi} A_{n+2}^{\mathrm{YM}}(1,2, \ldots, m, p,\{m+1, \ldots, n-1\} \amalg\{q\}, n) .
\end{aligned}
$$


Now putting (6.21) and (6.18) back to (6.15), we get

$$
\begin{aligned}
A_{n, 2}^{\mathrm{EYM}}(1,2, \ldots, n ; p, q)= & \sum_{ш} \frac{\left(k_{1} f_{p} Y_{p}\right)}{\left(k_{1} k_{p}\right)} \frac{\left(k_{1} f_{q} Y_{q}\right)}{\left(k_{1} k_{q}\right)} A_{n+2}^{\mathrm{YM}}(1,2,\{3, \ldots, n-1\} ш\{p\} ш\{q\}, n) \\
& +\sum_{ш} \frac{\left(k_{1} \cdot f_{p} \cdot k_{q}\right)}{\left(k_{1} \cdot k_{p}\right)} \frac{\left(k_{1} f_{q} Y_{q}\right)}{\left(k_{1} k_{q}\right)} A_{n+2}^{\mathrm{YM}}(1,2,\{3, \ldots, n-1\} ш\{q, p\}, n) \\
& +\sum_{ш} \frac{\left(k_{1} \cdot f_{q} \cdot k_{p}\right)}{\left(k_{1} \cdot k_{q}\right)} \frac{\left(k_{1} f_{p} Y_{p}\right)}{\left(k_{1} k_{p}\right)} A_{n+2}^{\mathrm{YM}}(1,2,\{3, \ldots, n-1\} ш\{p, q\}, n)+\frac{\left(k_{1} \cdot f_{p} \cdot f_{q} \cdot k_{1}\right)}{\left(k_{1} \cdot k_{p}\right)\left(k_{1} \cdot k_{q}\right)} D .
\end{aligned}
$$

(v) For the D: For the last one, we need the action keeping the last term. One of such a choice is $\mathcal{T}_{1 q p} \mathcal{T}_{1 p 2}$. Acting on (6.22), we get

$$
\begin{aligned}
\mathcal{L}= & A_{n+2}^{\mathrm{YM}}(1, q, p, 2, \ldots, n) \\
\mathcal{R}= & \sum_{\omega} \frac{\left(-k_{p} \cdot Y_{p}\right)}{\left(k_{1} k_{p}\right)} \frac{\left(-k_{q} \cdot\left(Y_{q}-k_{1}\right)\right)}{\left(k_{1} k_{q}\right)} A_{n+2}^{\mathrm{YM}}(1,2,\{3, \ldots, n-1\} ш\{p\} \omega\{q\}, n) \\
& +\sum_{\omega} \frac{\left(-k_{p} \cdot k_{q}\right)}{\left(k_{1} \cdot k_{p}\right)} \frac{\left(-k_{q} \cdot\left(Y_{q}-k_{1}\right)\right)}{\left(k_{1} k_{q}\right)} A_{n+2}^{\mathrm{YM}}(1,2,\{3, \ldots, n-1\} ш\{q, p\}, n) \\
& +\sum_{\omega} \frac{-\left(\left(k_{p}+k_{1}\right) \cdot k_{q}\right)}{\left(k_{1} \cdot k_{q}\right)} \frac{\left(-k_{p} Y_{p}\right)}{\left(k_{1} k_{p}\right)} A_{n+2}^{\mathrm{YM}}(1,2,\{3, \ldots, n-1\} \omega\{p, q\}, n)-\frac{k_{q} \cdot\left(k_{p}+k_{1}\right)}{\left(k_{1} \cdot k_{p}\right)\left(k_{1} \cdot k_{q}\right)} D
\end{aligned}
$$

where we have used (6.13). From it, we can solve that

$$
\begin{aligned}
\left(\left(k_{p}+k_{1}\right) \cdot k_{q}\right) D= & \sum_{ш}\left(k_{p} \cdot X_{p}\right)\left(k_{q} \cdot\left(Y_{q}-k_{1}\right)\right) A_{n+2}^{\mathrm{YM}}(1,2,\{3, \ldots, n-1\} ш\{q, p\}, n) \\
& +\sum_{ш}\left(k_{q} \cdot\left(k_{p}+Y_{q}\right)\right)\left(k_{p} Y_{p}\right) A_{n+2}^{\mathrm{YM}}(1,2,\{3, \ldots, n-1\} ш\{p, q\}, n) \\
& -\left(k_{1} \cdot k_{p}\right)\left(k_{1} \cdot k_{q}\right) A_{n+2}^{\mathrm{YM}}(1, q, p, 2, \ldots, n) .
\end{aligned}
$$

Now using (2.11) and with some algebraic simplifications, we find

$$
\begin{aligned}
D= & \frac{\left(K_{q} \cdot X_{q}\right)\left(k_{p} \cdot\left(Y_{p}-k_{1}\right)\right)}{\mathcal{K}_{1 p q}} A_{n+1}^{\mathrm{YM}}(1,2,\{3, \ldots, n-1\} ш\{p, q\}, n) \\
& +\frac{\left(K_{p} \cdot X_{p}\right)\left(k_{q} \cdot\left(Y_{q}-k_{1}\right)\right)}{\mathcal{K}_{1 p q}} A_{n+2}^{\mathrm{YM}}(1,2,\{3, \ldots, n-1\} ш\{q, p\}, n) .
\end{aligned}
$$

Putting back (6.25) to (6.22), we finally get

$$
\begin{aligned}
A_{n, 2}^{\mathrm{EYM}}(1,2, \ldots, n ; p, q)= & \sum_{ш} \frac{\left(k_{1} f_{p} Y_{p}\right)}{\left(k_{1} k_{p}\right)} \frac{\left(k_{1} f_{q} Y_{q}\right)}{\left(k_{1} k_{q}\right)} A_{n+2}^{\mathrm{YM}}(1,2,\{3, \ldots, n-1\} ш\{p\} ш\{q\}, n) \\
& +\sum_{ш} \frac{\left(k_{1} \cdot f_{p} \cdot k_{q}\right)}{\left(k_{1} \cdot k_{p}\right)} \frac{\left(k_{1} f_{q} Y_{q}\right)}{\left(k_{1} k_{q}\right)} A_{n+2}^{\mathrm{YM}}(1,2,\{3, \ldots, n-1\} ш\{q, p\}, n) \\
& +\sum_{w} \frac{\left(k_{1} \cdot f_{q} \cdot k_{p}\right)}{\left(k_{1} \cdot k_{q}\right)} \frac{\left(k_{1} f_{p} Y_{p}\right)}{\left(k_{1} k_{p}\right)} A_{n+2}^{\mathrm{YM}}(1,2,\{3, \ldots, n-1\} ш\{p, q\}, n) \\
& +\frac{\left(k_{1} \cdot f_{p} \cdot f_{q} \cdot k_{1}\right)}{\left(k_{1} \cdot k_{p}\right)\left(k_{1} \cdot k_{q}\right)}\left\{\frac{\left(K_{q} \cdot X_{q}\right)\left(k_{p} \cdot\left(Y_{p}-k_{1}\right)\right)}{\mathcal{K}_{1 p q}} A_{n+1}^{\mathrm{YM}}(1,2,\{3, \ldots, n-1\} ш\{p, q\}, n)\right. \\
& \left.+\frac{\left(K_{p} \cdot X_{p}\right)\left(k_{q} \cdot\left(Y_{q}-k_{1}\right)\right)}{\mathcal{K}_{1 p q}} A_{n+2}^{\mathrm{YM}}(1,2,\{3, \ldots, n-1\} ш\{q, p\}, n)\right\},
\end{aligned}
$$


which is nothing but the result (5.11). We want to emphasize again that using the differential operators on the gauge invariant building blocks, we reach the expansion in the BCJ basis naturally without any further preassumption. In the derivation, the symmetry between $p, q$ is automatically kept.

Before ending this part, let us give another remark. To solve $D$ we have used the differential operator $\mathcal{T}_{1 q p} \mathcal{T}_{1 p 2}$ and the result (2.11). However, we can use another operator $\mathcal{T}_{1 q 2} \mathcal{T}_{q p 2}$ to find $D$. If we act it on both sides of (6.22), we will get the same $\mathcal{L}$ and different $\mathcal{R}$ in (6.23). Using these two different expressions of $\mathcal{R}$, we can solve $D$. Putting the $D$ back, we get the $\mathcal{L}$, which is nothing but the expression (2.11). In other words, we have derived the expansion of YM amplitudes to its $\mathrm{BCJ}$ basis for this special case, just like the derivation of the fundamental BCJ relations in the previous subsection (6.10). From the new angle, the BCJ relations are consistent conditions of these differential operators. It is similar to the observation that consistent conditions for different KLT relations [17,39,40,45-48] will imply these BCJ relations.

\section{Another derivation}

The starting point of the previous derivation is the expansion (6.11) of $A_{n, 2}^{\mathrm{EYM}}$ using building blocks of two gravitons. Now we consider another derivation, which has the recursive structure like the expansion in the KK basis. We can first view $A_{n, 2}^{\text {EYM }}$ as the polynomial functions of $\epsilon_{p}$ and write its manifestly gauge invariant expansion by building blocks of $\epsilon_{p}$ as

$$
A_{n, 2}^{\mathrm{EYM}}(1, \ldots, n ; p, q)=\sum_{a=2}^{n-1} \frac{\left(k_{1} f_{p} K_{a}\right)}{\left(k_{1} k_{p}\right)} B_{a}+\frac{\left(k_{1} f_{p} k_{q}\right)}{\left(k_{1} k_{p}\right)} B_{q}+\frac{\left(k_{1} f_{p} f_{q} k_{1}\right)}{\left(k_{1} k_{p}\right)\left(k_{1} k_{q}\right)} E_{p q}
$$

with $B_{a}$ and $B_{q}$ being polynomials of $\epsilon_{q}$. Now we use differential operators to determine these unknown variables one by one:

(1) First, we use insertion operators $\mathcal{T}_{i p(i+1)}$ with $2 \leq i \leq n-1$ to determine $B_{a}$. After applying these operators, we get

$$
A_{n+1,1}^{\mathrm{EYM}}(1, \ldots, i, p, i+1, n ; q)=\sum_{a=2}^{n-1} \mathcal{T}_{i p(i+1)} \frac{\left(k_{1} f_{p} K_{a}\right)}{\left(k_{1} k_{p}\right)} B_{a}=B_{i} .
$$

(2) For the remaining two variables, there are no differential operators involving only graviton $p$ to select them one by one, and we need to expand it further according to $\epsilon_{q}$ as

$$
B_{q}=\sum_{b=2}^{n-1} \frac{\left(k_{1} f_{q} K_{b}\right)}{\left(k_{1} k_{q}\right)} B_{q b}+\frac{\left(k_{1} f_{q} k_{p}\right)}{\left(k_{1} k_{q}\right)} B_{q p}
$$

Now

$$
\begin{aligned}
A_{n, 2}^{\mathrm{EYM}}(1, \ldots, n ; p, q)= & \sum_{\omega} \frac{\left(k_{1} f_{p} Y_{p}\right)}{\left(k_{1} k_{p}\right)} A_{n+1,1}^{\mathrm{EYM}}(1,2,\{3, \ldots, n-1\} ш\{p\}, n ; q) \\
& +\sum_{b=2}^{n-1} \frac{\left(k_{1} f_{p} k_{q}\right)}{\left(k_{1} k_{p}\right)} \frac{\left(k_{1} f_{q} K_{b}\right)}{\left(k_{1} k_{q}\right)} B_{q b}+\frac{\left(k_{1} f_{p} k_{q}\right)}{\left(k_{1} k_{p}\right)} \frac{\left(k_{1} f_{q} k_{p}\right)}{\left(k_{1} k_{q}\right)} B_{q p}+\frac{\left(k_{1} f_{p} f_{q} k_{1}\right)}{\left(k_{1} k_{p}\right)\left(k_{1} k_{q}\right)} E_{p q} .
\end{aligned}
$$

Because of the index circle structure, we have immediately $B_{q p}=0$.

(3) Now we apply insertion operators $\mathcal{T}_{i q(i+1)} \mathcal{T}_{q p(i+1)}$ to the above equation to determine $B_{q b}$. We get

$$
A_{n+2}^{\mathrm{YM}}(1, \ldots, i, q, p, i+1, \ldots, n)=-\sum_{ш} A_{n+2}(1, \ldots, i, q, i+1,\{i+2, \ldots, n-1\} ш\{p\}, n)+B_{q b} .
$$

From it, we can solve

$$
B_{q b}=\sum_{\Psi} A_{n+2}(1, \ldots, i, q,\{i+1, \ldots, n-1\} ш\{p\}, n) .
$$


Thus, we can write

$$
\begin{aligned}
A_{n, 2}^{\mathrm{EYM}}(1, \ldots, n ; p, q)= & \sum_{\omega} \frac{\left(k_{1} f_{p} Y_{p}\right)}{\left(k_{1} k_{p}\right)} A_{n+1,1}^{\mathrm{EYM}}(1,2,\{3, \ldots, n-1\} ш\{p\}, n ; q) \\
& +\sum_{\omega} \frac{\left(k_{1} f_{p} k_{q}\right)}{\left(k_{1} k_{p}\right)} \frac{\left(k_{1} f_{q} Y_{q}\right)}{\left(k_{1} k_{q}\right)} A_{n+2}(1,2,\{3, \ldots, n-1\} \omega\{q, p\}, n)+\frac{\left(k_{1} f_{p} f_{q} k_{1}\right)}{\left(k_{1} k_{p}\right)\left(k_{1} k_{q}\right)} E_{p q} .
\end{aligned}
$$

(4) The determination of $E_{p q}$ will be exactly the same as the determination of $D$ in (6.25) and we will not repeat the calculation.

Assembling all pieces together, we get

$$
\begin{aligned}
A_{n, 2}^{\mathrm{EYM}}(1, \ldots, n ; p, q)= & \sum_{\omega} \frac{\left(k_{1} f_{p} X_{p}\right)}{\left(k_{1} k_{p}\right)} \frac{\left(k_{1} f_{q} X_{q}\right)}{\left(k_{1} k_{q}\right)} A_{n+2}(1,2,\{3, \ldots, n-1\} ш\{p\} ш\{q\}, n) \\
& +\frac{\left(k_{1} f_{p} f_{q} k_{1}\right)}{\left(k_{1} k_{p}\right)\left(k_{1} k_{q}\right)} \frac{\left[k_{p}\left(Y_{p}-k_{1}\right)\right]\left(k_{q} X_{q}\right)}{\mathcal{K}_{1 p q}} A_{n+2}^{\mathrm{YM}}(1,2,\{3, \ldots, n-1\} ш\{p, q\}, n) \\
& +\frac{\left(k_{1} f_{p} f_{q} k_{1}\right)}{\left(k_{1} k_{p}\right)\left(k_{1} k_{q}\right)} \frac{\left[k_{q}\left(Y_{q}-k_{1}\right)\right]\left(k_{p} X_{p}\right)}{\mathcal{K}_{1 p q}} A_{n+2}^{\mathrm{YM}}(1,2,\{3, \ldots, n-1\} \omega\{q, p\}, n) .
\end{aligned}
$$

\section{The case with three gravitons}

In this subsection, we consider a little more complicated case, i.e., the sEYM amplitudes with three gravitons. We will use the recursive structure to express the expansion.

First, we write down the expansion according to the manifestly gauge invariant building blocks of $\epsilon_{p}$ as

$A_{n, 3}^{\mathrm{EYM}}(1,2, \ldots, n ; p, q, r)=\sum_{a=2}^{n-1} \frac{\left(k_{1} f_{p} K_{a}\right)}{\left(k_{1} k_{p}\right)} B_{a}+\frac{\left(k_{1} f_{p} k_{q}\right)}{\left(k_{1} k_{p}\right)} B_{p}+\frac{\left(k_{1} f_{p} k_{r}\right)}{\left(k_{1} k_{p}\right)} B_{r}+\frac{\left(k_{1} f_{p} f_{q} k_{1}\right)}{\left(k_{1} k_{p}\right)\left(k_{1} k_{q}\right)} D_{p q}+\frac{\left(k_{1} f_{p} f_{r} k_{1}\right)}{\left(k_{1} k_{p}\right)\left(k_{1} k_{r}\right)} D_{p r}$

with $B$ 's being polynomials of $\epsilon_{q}$ and $\epsilon_{r}, D_{p q}$ of $\epsilon_{r}$ and $D_{p r}$ of $\epsilon_{q}$. Now we solve these coefficients step by step:

(1) It is clear that applying insertion operators $\mathcal{T}_{i p(i+1)}$ with $i=2, \ldots, n-1$ directly, we can solve

$$
\mathcal{T}_{i p(i+1)} A_{n, 3}^{\mathrm{EYM}}(1,2, \ldots, n ; p, q, r)=A_{n+1,2}^{\mathrm{EYM}}(1, \ldots, i, p, i+1, \ldots, n ; q, r)=\sum_{a=2}^{n-1} \mathcal{T}_{i p(i+1)} \frac{\left(k_{1} f_{p} K_{a}\right)}{\left(k_{1} k_{p}\right)} B_{a}=B_{i}
$$

(2) To determine $B_{p}$, we need to expand it further as

$$
\frac{\left(k_{1} f_{p} k_{q}\right)}{\left(k_{1} k_{p}\right)} B_{q}=\frac{\left(k_{1} f_{p} k_{q}\right)}{\left(k_{1} k_{p}\right)}\left\{\sum_{b=2}^{n-1} \frac{\left(k_{1} f_{q} K_{b}\right)}{\left(k_{1} k_{q}\right)} B_{q b}+\frac{\left(k_{1} f_{q} k_{p}\right)}{\left(k_{1} k_{q}\right)} B_{q p}+\frac{\left(k_{1} f_{q} k_{r}\right)}{\left(k_{1} k_{q}\right)} B_{q r}+\frac{\left(k_{1} f_{q} f_{r} k_{1}\right)}{\left(k_{1} k_{q}\right)\left(k_{1} k_{r}\right)} D_{q q r}\right\}
$$

Because of the index circle structure, $B_{q p}=0$. Next we compute coefficients $B_{q b}$ and $B_{q r}$, and leave $D_{q q r}$ to later. Applying the insertion operator $\mathcal{T}_{i q(i+1)} \mathcal{T}_{q p(i+1)}$, we have

$$
A_{n+2,1}^{\mathrm{EYM}}(1, \ldots, i, q, p, i+1, \ldots, n ; r)=-\sum_{ш} A_{n+2,1}(1, \ldots, i, q, i+1,\{i+2, \ldots, n-1\} ш\{p\}, n ; r)+B_{q i},
$$

from which we solve 


$$
B_{q i}=\sum_{\varpi} A_{n+2,1}(1, \ldots, i, q,\{i+1, \ldots, n-1\} ш\{p\}, n ; r) .
$$

To determine $B_{q r}$, we need to expand it further

$$
\frac{\left(k_{1} f_{p} k_{q}\right)}{\left(k_{1} k_{p}\right)} \frac{\left(k_{1} f_{q} k_{r}\right)}{\left(k_{1} k_{q}\right)} B_{q r}=\frac{\left(k_{1} f_{p} k_{q}\right)}{\left(k_{1} k_{p}\right)} \frac{\left(k_{1} f_{q} k_{r}\right)}{\left(k_{1} k_{q}\right)}\left\{\sum_{c=2}^{n-1} \frac{\left(k_{1} f_{r} K_{c}\right)}{\left(k_{1} k_{r}\right)} B_{q r c}+\frac{\left(k_{1} f_{r} k_{p}\right)}{\left(k_{1} k_{r}\right)} B_{q r p}+\frac{\left(k_{1} f_{r} k_{q}\right)}{\left(k_{1} k_{r}\right)} B_{q r q}\right\} .
$$

Again because of the index circle structure, $B_{q r p}=B_{q r q}=0$. We apply the insertion operator $\mathcal{T}_{i q(i+1)} \mathcal{T}_{r q(i+1)} \mathcal{T}_{q p(i+1)}$ to get

$$
\begin{aligned}
A_{n+3}(1, \ldots, i, r, q, p, i+1, \ldots, n)= & -\sum_{\varpi} A_{n+3}(1, \ldots, i, r, q, i+1,\{i+2, \ldots, n-1\} ш\{p\}, n) \\
& -\sum_{\varpi} A_{n+3}(1, \ldots, i, r, i+1,\{i+2, \ldots, n-1\} \amalg\{q, p\}, n)+B_{q r i},
\end{aligned}
$$

so $B_{q r c}$ is solved to be

$$
B_{q r i}=\sum_{\uplus} A_{n+3}(1, \ldots, i, r,\{i+1, \ldots, n-1\} ш\{q, p\}, n) .
$$

(3) As for the coefficient $B_{r}$, because of the symmetry between the $q$ and $r$, we can take the exact same steps as before and get similar results

$$
\begin{aligned}
\frac{\left(k_{1} f_{p} k_{r}\right)}{\left(k_{1} k_{p}\right)} B_{r}= & \sum_{\Psi} \frac{\left(k_{1} f_{p} k_{r}\right)}{\left(k_{1} k_{p}\right)} \frac{\left(k_{1} f_{r} Y_{r}\right)}{\left(k_{1} k_{r}\right)} A_{n+2,1}(1,2,\{3, \ldots, n-1\} \omega\{r, p\}, n ; q) \\
& +\sum_{\Psi} \frac{\left(k_{1} f_{p} k_{r}\right)}{\left(k_{1} k_{p}\right)} \frac{\left(k_{1} f_{r} k_{q}\right)}{\left(k_{1} k_{r}\right)} \frac{\left(k_{1} f_{q} Y_{q}\right)}{\left(k_{1} k_{r}\right)} A_{n+3}(1,2,\{3, \ldots, n-1\} ш\{q, r, p\}, n) \\
& +\frac{\left(k_{1} f_{p} k_{r}\right)}{\left(k_{1} k_{p}\right)} \frac{\left(k_{1} f_{r} f_{q} k_{1}\right)}{\left(k_{1} k_{q}\right)\left(k_{1} k_{r}\right)} D_{r q r} .
\end{aligned}
$$

(4) Up to now, we have

$$
\begin{aligned}
A_{n, 3}^{\mathrm{EYM}}(1,2, \ldots, n ; p, q, r)= & \sum_{\Psi} \frac{\left(k_{1} f_{p} Y_{p}\right)}{\left(k_{1} k_{p}\right)} A_{n+1,2}^{\mathrm{EYM}}(1,2,\{3, \ldots, n-1\} ш\{p\}, n ; q, r) \\
& +\sum_{\Psi} \frac{\left(k_{1} f_{p} k_{q}\right)}{\left(k_{1} k_{p}\right)} \frac{\left(k_{1} f_{q} Y_{q}\right)}{\left(k_{1} k_{q}\right)} A_{n+2,1}(1,2,\{3, \ldots, n-1\} ш\{q, p\}, n ; r) \\
& +\sum_{\Psi} \frac{\left(k_{1} f_{p} k_{q}\right)}{\left(k_{1} k_{p}\right)} \frac{\left(k_{1} f_{q} k_{r}\right)}{\left(k_{1} k_{q}\right)} \frac{\left(k_{1} f_{r} Y_{r}\right)}{\left(k_{1} k_{r}\right)} A_{n+3}(1,2,\{3, \ldots, n-1\} ш\{r, q, p\}, n) \\
& +\sum_{\Psi} \frac{\left(k_{1} f_{p} k_{r}\right)}{\left(k_{1} k_{p}\right)} \frac{\left(k_{1} f_{r} Y_{r}\right)}{\left(k_{1} k_{r}\right)} A_{n+2,1}(1,2,\{3, \ldots, n-1\} ш\{r, p\}, n ; q) \\
& +\sum_{\Psi} \frac{\left(k_{1} f_{p} k_{r}\right)}{\left(k_{1} k_{p}\right)} \frac{\left(k_{1} f_{r} k_{q}\right)}{\left(k_{1} k_{r}\right)} \frac{\left(k_{1} f_{q} Y_{q}\right)}{\left(k_{1} k_{r}\right)} A_{n+3}(1,2,\{3, \ldots, n-1\} ш\{q, r, p\}, n) \\
& +\frac{\left(k_{1} f_{p} k_{r}\right)}{\left(k_{1} k_{p}\right)} \frac{\left(k_{1} f_{r} f_{q} k_{1}\right)}{\left(k_{1} k_{q}\right)\left(k_{1} k_{r}\right)} D_{r q r}+\frac{\left(k_{1} f_{p} k_{q}\right)}{\left(k_{1} k_{p}\right)} \frac{\left(k_{1} f_{q} f_{r} k_{1}\right)}{\left(k_{1} k_{q}\right)\left(k_{1} k_{r}\right)} D_{q q r} \\
& +\frac{\left(k_{1} f_{p} f_{q} k_{1}\right)}{\left(k_{1} k_{p}\right)\left(k_{1} k_{q}\right)} D_{p q}+\frac{\left(k_{1} f_{p} f_{r} k_{1}\right)}{\left(k_{1} k_{p}\right)\left(k_{1} k_{r}\right)} D_{p r} .
\end{aligned}
$$


(5) Now we consider these left $D$ 's. First we expand $D_{p q}$ as

$$
\frac{\left(k_{1} f_{p} f_{q} k_{1}\right)}{\left(k_{1} k_{p}\right)\left(k_{1} k_{q}\right)} D_{p q}=\frac{\left(k_{1} f_{p} f_{q} k_{1}\right)}{\left(k_{1} k_{p}\right)\left(k_{1} k_{q}\right)}\left\{\sum_{c=2}^{n-1} \frac{\left(k_{1} f_{r} K_{c}\right)}{\left(k_{1} k_{r}\right)} D_{p q c}+\frac{\left(k_{1} f_{r} k_{p}\right)}{\left(k_{1} k_{r}\right)} D_{p q p}+\frac{\left(k_{1} f_{r} k_{q}\right)}{\left(k_{1} k_{r}\right)} D_{p q q}\right\} .
$$

Now, applying insertion operators $\mathcal{T}_{i r(i+1)} \mathcal{T}_{1 q 2} \mathcal{T}_{q p 2}$ with $2 \leq i \leq n-1$ we get

$$
\begin{aligned}
A_{n+3}(1, q, p, 2, \ldots, i, r, i+1, \ldots, n)= & -\sum_{\Psi} A_{n+3}(1, q, 2,\{3, \ldots, i, r, i+1, \ldots, n-1\} ш\{p\}, n) \\
& -\sum_{\Psi} \frac{\left(k_{q} Y_{q}\right)}{\left(k_{1} k_{q}\right)} A_{n+3}(1,2,\{3, \ldots, i, r, i+1, \ldots, n-1\} ш\{q, p\}, n) \\
& -\sum_{\Psi} \frac{\left(k_{r} Y_{r}\right)}{\left(k_{1} k_{r}\right)} A_{n+3}(1,2, \ldots, i, r,\{i+1, \ldots, n-1\} ш\{q, p\}, n)+\frac{1}{\left(k_{1} k_{q}\right)} D_{p q i} .
\end{aligned}
$$

Solving it in the BCJ basis of YM amplitudes, we find

$$
\begin{aligned}
D_{p q i}= & \frac{\left[k_{q}\left(X_{q}-k_{1}\right)\right]\left(k_{p} X_{p}\right)}{\mathcal{K}_{1 p q}} A_{n+3}(1,2,\{3, \ldots, i, r, i+1, \ldots, n-1\} ш\{q, p\}, n) \\
& +\frac{\left[k_{p}\left(X_{p}-k_{1}\right)\right]\left(k_{q} X_{q}\right)}{\mathcal{K}_{1 p q}} A_{n+3}(1,2,\{3, \ldots, i, r, i+1, \ldots, n-1\} ш\{p, q\}, n) .
\end{aligned}
$$

Similarly by the symmetry between $q$ and $r$, after expanding $D_{p r}$ and applying $\mathcal{T}_{i q(i+1)} \mathcal{T}_{1 r 2} \mathcal{T}_{r p 2}$, we can write

$$
\begin{aligned}
\frac{\left(k_{1} f_{p} f_{r} k_{1}\right)}{\left(k_{1} k_{p}\right)\left(k_{1} k_{r}\right)} D_{p r}= & \sum_{\varpi} \frac{\left(k_{1} f_{p} f_{r} k_{1}\right)}{\left(k_{1} k_{p}\right)\left(k_{1} k_{r}\right)} \frac{\left(k_{1} f_{q} Y_{q}\right)}{\left(k_{1} k_{q}\right)}\left\{\frac{\left[k_{r}\left(X_{r}-k_{1}\right)\right]\left(k_{p} X_{p}\right)}{\mathcal{K}_{1 p r}} A_{n+3}(1,2,\{3, \ldots, n-1\} ш\{q\} ш\{r, p\}, n)\right. \\
& \left.+\frac{\left[k_{p}\left(X_{p}-k_{1}\right)\right]\left(k_{r} X_{r}\right)}{\mathcal{K}_{1 p r}} A_{n+3}(1,2,\{3, \ldots, n-1\} \omega\{q\} ш\{p, r\}, n)\right\} \\
& +\frac{\left(k_{1} f_{p} f_{r} k_{1}\right)}{\left(k_{1} k_{p}\right)\left(k_{1} k_{r}\right)} \frac{\left(k_{1} f_{q} k_{p}\right)}{\left(k_{1} k_{q}\right)} D_{p r p}+\frac{\left(k_{1} f_{p} f_{r} k_{1}\right)}{\left(k_{1} k_{p}\right)\left(k_{1} k_{r}\right)} \frac{\left(k_{1} f_{q} k_{r}\right)}{\left(k_{1} k_{q}\right)} D_{p r r} .
\end{aligned}
$$

Up to now the expansion of $A_{n, 3}^{\mathrm{EYM}}$ is given by

$$
\begin{aligned}
& A_{n, 3}^{\mathrm{EYM}}(1,2, \ldots, n ; p, q, r) \\
& =\sum_{\Psi} \frac{\left(k_{1} f_{p} Y_{p}\right)}{\left(k_{1} k_{p}\right)} A_{n+1,2}^{\mathrm{EYM}}(1,2,\{3, \ldots, n-1\} ш\{p\}, n ; q, r) \\
& +\sum_{\Psi} \frac{\left(k_{1} f_{p} k_{q}\right)}{\left(k_{1} k_{p}\right)} \frac{\left(k_{1} f_{q} Y_{q}\right)}{\left(k_{1} k_{q}\right)} A_{n+2,1}(1,2,\{3, \ldots, n-1\} ш\{q, p\}, n ; r) \\
& +\sum_{ш} \frac{\left(k_{1} f_{p} k_{q}\right)}{\left(k_{1} k_{p}\right)} \frac{\left(k_{1} f_{q} k_{r}\right)}{\left(k_{1} k_{q}\right)} \frac{\left(k_{1} f_{r} Y_{r}\right)}{\left(k_{1} k_{r}\right)} A_{n+3}(1,2,\{3, \ldots, n-1\} ш\{r, q, p\}, n) \\
& +\sum_{\amalg} \frac{\left(k_{1} f_{p} k_{r}\right)}{\left(k_{1} k_{p}\right)} \frac{\left(k_{1} f_{r} Y_{r}\right)}{\left(k_{1} k_{r}\right)} A_{n+2,1}(1,2,\{3, \ldots, n-1\} \amalg\{r, p\}, n ; q) \\
& +\sum_{\varpi} \frac{\left(k_{1} f_{p} k_{r}\right)}{\left(k_{1} k_{p}\right)} \frac{\left(k_{1} f_{r} k_{q}\right)}{\left(k_{1} k_{r}\right)} \frac{\left(k_{1} f_{q} Y_{q}\right)}{\left(k_{1} k_{r}\right)} A_{n+3}(1,2,\{3, \ldots, n-1\} ш\{q, r, p\}, n)
\end{aligned}
$$




$$
\begin{aligned}
& +\sum_{\amalg} \frac{\left(k_{1} f_{p} f_{q} k_{1}\right)}{\left(k_{1} k_{p}\right)\left(k_{1} k_{q}\right)} \frac{\left(k_{1} f_{r} Y_{r}\right)}{\left(k_{1} k_{r}\right)}\left\{\frac{\left[k_{q}\left(X_{q}-k_{1}\right)\right]\left(k_{p} X_{p}\right)}{\mathcal{K}_{1 p q}} A_{n+3}(1,2,\{3, \ldots, n-1\} ш\{r\} ш\{q, p\}, n)\right. \\
& \left.+\frac{\left[k_{p}\left(X_{p}-k_{1}\right)\right]\left(k_{q} X_{q}\right)}{\mathcal{K}_{1 p q}} A_{n+3}(1,2,\{3, \ldots, n-1\} ш\{r\} \omega\{p, q\}, n)\right\} \\
& +\sum_{\amalg} \frac{\left(k_{1} f_{p} f_{r} k_{1}\right)}{\left(k_{1} k_{p}\right)\left(k_{1} k_{r}\right)} \frac{\left(k_{1} f_{q} Y_{q}\right)}{\left(k_{1} k_{q}\right)}\left\{\frac{\left[k_{r}\left(X_{r}-k_{1}\right)\right]\left(k_{p} X_{p}\right)}{\mathcal{K}_{1 p r}} A_{n+3}(1,2,\{3, \ldots, n-1\} ш\{q\} \amalg\{r, p\}, n)\right. \\
& \left.+\frac{\left[k_{p}\left(X_{p}-k_{1}\right)\right]\left(k_{r} X_{r}\right)}{\mathcal{K}_{1 p r}} A_{n+3}(1,2,\{3, \ldots, n-1\} ш\{q\} ш\{p, r\}, n)\right\} \\
& +\frac{\left(k_{1} f_{p} k_{r}\right)}{\left(k_{1} k_{p}\right)} \frac{\left(k_{1} f_{r} f_{q} k_{1}\right)}{\left(k_{1} k_{q}\right)\left(k_{1} k_{r}\right)} D_{r q r}+\frac{\left(k_{1} f_{p} k_{q}\right)}{\left(k_{1} k_{p}\right)} \frac{\left(k_{1} f_{q} f_{r} k_{1}\right)}{\left(k_{1} k_{q}\right)\left(k_{1} k_{r}\right)} D_{q q r}+\frac{\left(k_{1} f_{p} f_{q} k_{1}\right)}{\left(k_{1} k_{p}\right)\left(k_{1} k_{q}\right)} \frac{\left(k_{1} f_{r} k_{p}\right)}{\left(k_{1} k_{r}\right)} D_{p q p} \\
& +\frac{\left(k_{1} f_{p} f_{q} k_{1}\right)}{\left(k_{1} k_{p}\right)\left(k_{1} k_{q}\right)} \frac{\left(k_{1} f_{r} k_{q}\right)}{\left(k_{1} k_{r}\right)} D_{p q q}+\frac{\left(k_{1} f_{p} f_{r} k_{1}\right)}{\left(k_{1} k_{p}\right)\left(k_{1} k_{r}\right)} \frac{\left(k_{1} f_{q} k_{p}\right)}{\left(k_{1} k_{q}\right)} D_{p r p}+\frac{\left(k_{1} f_{p} f_{r} k_{1}\right)}{\left(k_{1} k_{p}\right)\left(k_{1} k_{r}\right)} \frac{\left(k_{1} f_{q} k_{r}\right)}{\left(k_{1} k_{q}\right)} D_{p r r} .
\end{aligned}
$$

(6) Finally, we need to determine the six left $D$ 's. We rewrite these six terms as

$$
\begin{aligned}
C_{1}= & a_{1} \frac{\left(k_{1} f_{p} k_{q}\right)}{\mathcal{K}_{1 p}} \frac{\left(k_{1} f_{q} f_{r} k_{1}\right)}{\mathcal{K}_{1 q} \mathcal{K}_{1 r}}+a_{2} \frac{\left(k_{1} f_{p} k_{r}\right)}{\mathcal{K}_{1 p}} \frac{\left(k_{1} f_{r} f_{q} k_{1}\right)}{\mathcal{K}_{1 q} \mathcal{K}_{1 r}}+a_{3} \frac{\left(k_{1} f_{q} k_{p}\right)}{\mathcal{K}_{1 q}} \frac{\left(k_{1} f_{p} f_{r} k_{1}\right)}{\mathcal{K}_{1 p} \mathcal{K}_{1 r}} \\
& +a_{4} \frac{\left(k_{1} f_{q} k_{r}\right)}{\mathcal{K}_{1 q}} \frac{\left(k_{1} f_{r} f_{p} k_{1}\right)}{\mathcal{K}_{1 p} \mathcal{K}_{1 r}}+a_{5} \frac{\left(k_{1} f_{r} k_{p}\right)}{\mathcal{K}_{1 r}} \frac{\left(k_{1} f_{p} f_{q} k_{1}\right)}{\mathcal{K}_{1 p} \mathcal{K}_{1 q}}+a_{6} \frac{\left(k_{1} f_{r} k_{q}\right)}{\mathcal{K}_{1 r}} \frac{\left(k_{1} f_{q} f_{p} k_{1}\right)}{\mathcal{K}_{1 p} \mathcal{K}_{1 q}} \\
= & \sum_{\rho} a(\rho) \frac{\left(k_{1} f_{\rho_{3}} k_{\rho_{2}}\right)}{\mathcal{K}_{1 \rho_{3}}} \frac{\left(k_{1} f_{\rho_{2}} f_{\rho_{1}} k_{1}\right)}{\mathcal{K}_{1 \rho_{2}} \mathcal{K}_{1 \rho_{1}}}
\end{aligned}
$$

where $\rho$ is a permutation of $\{p, q, r\}$, and the other known terms in the expansion of $A_{n, 3}^{\mathrm{EYM}}$ are denoted by $C_{0}$. To determine them, we need to use the operators $\mathcal{T}_{1\{\rho\} 2} \equiv \mathcal{T}_{1 \rho_{1} \rho_{2} \rho_{3} 2}=\mathcal{T}_{\rho_{2} \rho_{3} 2} \mathcal{T}_{\rho_{1} \rho_{2} 2} \mathcal{T}_{1 \rho_{1} 2}$, whose effect is to insert $\{p, q, r\}$ between 1 and 2 consecutively with the ordering $\left(\rho_{1}, \rho_{2}, \rho_{3}\right)$. Applying $\mathcal{T}_{1\left\{\rho^{\prime}\right\} 2}$ to (6.39), the left-hand side gives

$$
\begin{aligned}
\mathcal{T}_{1\left\{\rho^{\prime}\right\}} A_{n, 3}^{\mathrm{EYM}}(1,2, \ldots, n ; p, q, r) & =A_{n+3}^{\mathrm{YM}}\left(1, \rho_{1}^{\prime}, \rho_{2}^{\prime}, \rho_{3}^{\prime}, 2, \ldots, n\right) \\
& =\sum_{\rho} \sum_{\varpi} \mathcal{C}^{\left(\rho^{\prime}\right)}(\rho, ш) A_{n+3}^{\mathrm{YM}}\left(1,2,\{3, \ldots, n-1\} ш\left\{\rho_{1}, \rho_{2}, \rho_{3}\right\}, n\right),
\end{aligned}
$$

where the expansions of general color ordered YM amplitudes to their BCJ basis have been used [see (2.21)]. The right-hand side gives

$$
\mathcal{T}_{1\left\{\rho^{\prime}\right\}_{2}}\left(C_{0}+C_{1}\right)=\mathcal{T}_{1\left\{\rho^{\prime}\right\} 2} C_{0}+\mathcal{T}_{1\left\{\rho^{\prime}\right\} 2} \sum_{\rho} a(\rho) \frac{\left(k_{1} f_{\rho_{3}} k_{\rho_{2}}\right)}{\mathcal{K}_{1 \rho_{3}}} \frac{\left(k_{1} f_{\rho_{2}} f_{\rho_{1}} k_{1}\right)}{\mathcal{K}_{1 \rho_{2}} \mathcal{K}_{1 \rho_{1}}}=\mathcal{T}_{1\left\{\rho^{\prime}\right\} 2} C_{0}+\frac{a\left(\rho^{\prime}\right)}{\left(k_{1} k_{\rho_{1}}\right)},
$$

where we have used the result $\mathcal{T}_{1\left\{\rho^{\prime}\right\} 2} \frac{\left(k_{1} \rho_{\rho_{3}} k_{\rho_{2}}\right.}{\mathcal{K}_{1 \rho_{3}}} \frac{\left(k_{1} f_{\rho_{2}} f_{\rho_{1}} k_{1}\right)}{\mathcal{K}_{1 \rho_{2}} \mathcal{K}_{1 \rho_{1}}}=\delta_{\rho, \rho^{\prime}} \frac{1}{\left(k_{1} k_{\rho_{1}}\right.}$. Comparing two sides we get

$$
a\left(\rho^{\prime}\right)=-\left(k_{1} k_{\rho_{1}}\right) \mathcal{T}_{1\left\{\rho^{\prime}\right\} 2} C_{0}+\left(k_{1} k_{\rho_{1}}\right) \sum_{\rho} \sum_{\Psi} \mathcal{C}^{\left(\rho^{\prime}\right)}(\rho, ш) A_{n+3}^{\mathrm{YM}}\left(1,2,\{3, \ldots, n-1\} ш\left\{\rho_{1}, \rho_{2}, \rho_{3}\right\}, n\right) .
$$

Having the general formula for $a\left(\rho^{\prime}\right)$ 's, we can calculate their expressions explicitly. To do so, first we rewrite $C_{0}$ as

$$
C_{0}=\sum_{\rho} \sum_{ш} C_{0}(\rho, ш) A_{n+3}^{\mathrm{YM}}\left(1,2,\{3, \ldots, n-1\} ш\left\{\rho_{1}, \rho_{2}, \rho_{3}\right\}, n\right),
$$


with

$$
\begin{aligned}
C_{0}(\rho, w)= & \frac{\left(k_{1} f_{\rho_{1}} X_{\rho_{1}}\right)}{\mathcal{K}_{1 \rho_{1}}} \frac{\left(k_{1} f_{\rho_{2}} X_{\rho_{2}}\right)}{\mathcal{K}_{1 \rho_{2}}} \frac{\left(k_{1} f_{\rho_{3}} X_{\rho_{3}}\right)}{\mathcal{K}_{1 \rho_{3}}}+\frac{\left[k_{\rho_{2}}\left(X_{\rho_{2}}-k_{1}\right)\right]\left(k_{\rho_{3}} X_{\rho_{3}}\right)}{\mathcal{K}_{1 \rho_{2} \rho_{3}}} \frac{\left(k_{1} f_{\rho_{1}} Y_{\rho_{1}}\right)}{\mathcal{K}_{1 \rho_{1}}} \frac{\left(k_{1} f_{\rho_{2}} f_{\rho_{3}} k_{1}\right)}{\mathcal{K}_{1 \rho_{2}} \mathcal{K}_{1 \rho_{3}}} \\
& +\frac{\left[k_{\rho_{1}}\left(X_{\rho_{1}}-k_{1}\right)\right]\left(k_{\rho_{3}} X_{\rho_{3}}\right)}{\mathcal{K}_{1 \rho_{1} \rho_{3}}} \frac{\left(k_{1} f_{\rho_{2}} Y_{\rho_{2}}\right)}{\mathcal{K}_{1 \rho_{2}}} \frac{\left(k_{1} f_{\rho_{1}} f_{\rho_{3}} k_{1}\right)}{\mathcal{K}_{1 \rho_{1}} \mathcal{K}_{1 \rho_{3}}}+\frac{\left[k_{\rho_{1}}\left(X_{\rho_{1}}-k_{1}\right)\right]\left(k_{\rho_{2}} X_{\rho_{2}}\right)}{\mathcal{K}_{1 \rho_{1} \rho_{2}}} \frac{\left(k_{1} f_{\rho_{3}} Y_{\rho_{3}}\right)}{\mathcal{K}_{1 \rho_{3}}} \frac{\left(k_{1} f_{\rho_{1}} f_{\rho_{2}} k_{1}\right)}{\mathcal{K}_{1 \rho_{1}} \mathcal{K}_{1 \rho_{2}}},
\end{aligned}
$$

then $a\left(\rho^{\prime}\right)$ becomes

$$
\begin{aligned}
a\left(\rho^{\prime}\right) & =\sum_{\rho} \sum_{\Psi}\left\{-\left(k_{1} k_{\rho_{1}}\right) \mathcal{T}_{1\left\{\rho^{\prime}\right\} 2} C_{0}(\rho, ш)+\left(k_{1} k_{\rho_{1}}\right) \mathcal{C}^{\left(\rho^{\prime}\right)}(\rho, ш)\right\} A_{n+3}^{\mathrm{YM}}\left(1,2,\{3, \ldots, n-1\} ш\left\{\rho_{1}, \rho_{2}, \rho_{3}\right\}, n\right) \\
& =\sum_{\rho} \sum_{\Psi} a\left(\rho^{\prime}, \rho\right) A_{n+3}^{\mathrm{YM}}\left(1,2,\{3, \ldots, n-1\} \amalg\left\{\rho_{1}, \rho_{2}, \rho_{3}\right\}, n\right),
\end{aligned}
$$

with

$$
a\left(\rho^{\prime}, \rho\right)=-\left(k_{1} k_{\rho_{1}}\right) \mathcal{T}_{1\left\{\rho^{\prime}\right\} 2} C_{0}(\rho, ш)+\left(k_{1} k_{\rho_{1}}\right) \mathcal{C}^{\left(\rho^{\prime}\right)}(\rho, ш) .
$$

After substituting $a\left(\rho^{\prime}, \rho\right)$ into (6.39), we get the final expansion of $A_{n, 3}^{\mathrm{EYM}}$ amplitudes in the BCJ basis

$$
A_{n, 3}^{\mathrm{EYM}}=\sum_{\rho} \sum_{\Psi} C(\rho, ш) A_{n+3}^{\mathrm{YM}}\left(1,2,\{3, \ldots, n-1\} ш\left\{\rho_{1}, \rho_{2}, \rho_{3}\right\}, n\right)
$$

with

$$
C(\rho, ш)=C_{0}(\rho, ш)+\sum_{\rho^{\prime}} a\left(\rho^{\prime}, \rho\right)
$$

Although (6.46) is the final result, to compare with (5.41) given in the previous section, we give the explicit expression of coefficients with the ordering $\rho=(p, q, r)$. Because $C_{0}(\rho, \omega)$ has been given, we only need to calculate $a\left(\rho^{\prime}, \rho\right)$. We have left the calculation details in Appendix $C$ and just quote the result of $C(\{p q r\}, \omega)$

$$
\begin{aligned}
& C(\{p q r\}, ш)= C_{0}(\{p q r\}, w)+C_{1}(\{p q r\}, w) \\
&= \frac{\left(k_{1} f_{p} X_{p}\right)}{\mathcal{K}_{1 p}} \frac{\left(k_{1} f_{q} X_{q}\right)}{\mathcal{K}_{1 q}} \frac{\left(k_{1} f_{r} X_{r}\right)}{\mathcal{K}_{1 r}}+\frac{\left[k_{q}\left(X_{q}-k_{1}\right)\right]\left(k_{r} X_{r}\right)}{\mathcal{K}_{1 q r}} \frac{\left(k_{1} f_{p} X_{p}\right)}{\mathcal{K}_{1 p}} \frac{\left(k_{1} f_{q} f_{r} k_{1}\right)}{\mathcal{K}_{1 q} \mathcal{K}_{1 r}} \\
&+\frac{\left[k_{p}\left(X_{p}-k_{1}\right)\right]\left(k_{r} X_{r}\right)}{\mathcal{K}_{1 p r}} \frac{\left(k_{1} f_{q} X_{q}\right)}{\mathcal{K}_{1 q}} \frac{\left(k_{1} f_{p} f_{r} k_{1}\right)}{\mathcal{K}_{1 p} \mathcal{K}_{1 r}}+\frac{\left[k_{p}\left(X_{p}-k_{1}\right)\right]\left(k_{q} X_{q}\right)}{\mathcal{K}_{1 p q}} \frac{\left(k_{1} f_{r} X_{r}\right)}{\mathcal{K}_{1 r}} \frac{\left(k_{1} f_{p} f_{q} k_{1}\right)}{\mathcal{K}_{1 p} \mathcal{K}_{1 q}} \\
&-\frac{\left(k_{r} X_{r}\right)\left[k_{q}\left(X_{q}-k_{1}\right)+\mathcal{K}_{1 q r}\right]\left[k_{p}\left(Y_{p}-k_{1}\right)\right]}{\mathcal{K}_{1 q r} \mathcal{K}_{1 p q r}} \frac{\left(k_{1} f_{p} k_{q}\right)}{\mathcal{K}_{1 p}} \frac{\left(k_{1} f_{q} f_{r} k_{1}\right)}{\mathcal{K}_{1 q} \mathcal{K}_{1 r}}-\frac{\left[k_{q}\left(X_{q}-k_{1}\right)\right]\left(k_{r} X_{r}\right)\left[k_{p}\left(Y_{p}-k_{1}\right)\right]}{\mathcal{K}_{1 q r} \mathcal{K}_{1 p q r}} \\
& \times \frac{\left(k_{1} f_{p} k_{r}\right)}{\mathcal{K}_{1 p}} \frac{\left(k_{1} f_{r} f_{q} k_{1}\right)}{\mathcal{K}_{1 q} \mathcal{K}_{1 r}}-\frac{\left(k_{r} X_{r}\right)\left[k_{p}\left(Y_{p}-k_{1}\right)\right]\left[k_{q}\left(X_{q}+k_{r}\right)\right]}{\mathcal{K}_{1 p r} \mathcal{K}_{1 p q r}} \frac{\left(k_{1} f_{q} k_{p}\right)}{\mathcal{K}_{1 q}} \frac{\left(k_{1} f_{p} f_{r} k_{1}\right)}{\mathcal{K}_{1 p} \mathcal{K}_{1 r}} \\
&-\frac{\left[k_{p}\left(Y_{p}-k_{1}\right)\right]\left(k_{r} X_{r}\right)\left[k_{1}\left(Y_{q}-k_{1}\right)\right]}{\mathcal{K}_{1 p r} \mathcal{K}_{1 p q r}} \frac{\left(k_{1} f_{q} k_{r}\right)}{\mathcal{K}_{1 q}} \frac{\left(k_{1} f_{r} f_{p} k_{1}\right)}{\mathcal{K}_{1 p} \mathcal{K}_{1 r}}-\frac{\left(k_{q} X_{q}\right)\left[k_{p}\left(Y_{p}-k_{1}\right)\right]\left(k_{r} X_{r}\right)}{\mathcal{K}_{1 p q} \mathcal{K}_{1 p q r}} \\
& \times \frac{\left(k_{1} f_{r} k_{p}\right)}{\mathcal{K}_{1 r}} \frac{\left(k_{1} f_{p} f_{q} k_{1}\right)}{\mathcal{K}_{1 p} \mathcal{K}_{1 q}}-\frac{\left[k_{p}\left(Y_{p}-k_{1}\right)\right]\left[\left(k_{q} X_{q}\right)-\mathcal{K}_{1 p q}\right]\left(k_{r} X_{r}\right)}{\mathcal{K}_{1 p q} \mathcal{K}_{1 p q r}} \frac{\left(k_{1} f_{r} k_{q}\right)\left(k_{1} f_{q} f_{p} k_{1}\right)}{\mathcal{K}_{1 r}} \\
& \mathcal{K}_{1 p} \mathcal{K}_{1 q}
\end{aligned}
$$

which is the same as the one given in the last section.

Similar to the previous two subsections, we see that to reach $A_{n+3}^{\mathrm{YM}}\left(1, \rho_{1}, \rho_{2}, \rho_{3}, 2, \ldots, n\right)$ with the fixed ordering $\rho$, there are five different insertion operators, i.e., 


$$
\begin{array}{ll}
\mathcal{T}_{1 \rho_{1} 2} \mathcal{T}_{\rho_{1} \rho_{3} 2} \mathcal{T}_{\rho_{1} \rho_{2} \rho_{3}}, & \mathcal{T}_{1 \rho_{1} 2} \mathcal{T}_{\rho_{1} \rho_{2} 2} \mathcal{T}_{\rho_{2} \rho_{3} 2} \\
\mathcal{T}_{1 \rho_{2} 2} \mathcal{T}_{1 \rho_{1} \rho_{2}} \mathcal{T}_{\rho_{2} \rho_{3} 2} & \\
\mathcal{T}_{1 \rho_{3} 2} \mathcal{T}_{1 \rho_{1} \rho_{3}} \mathcal{T}_{\rho_{1} \rho_{2} \rho_{3}}, & \mathcal{T}_{1 \rho_{3} 2} \mathcal{T}_{1 \rho_{2} \rho_{3}} \mathcal{T}_{1 \rho_{1} \rho_{2}}
\end{array}
$$

which can be used to act on the left-hand side of (6.39). With six orderings of $\rho$, we will get 29 equations after identifying the resulted right-hand sides of (6.39). Although we have not explicitly checked, we believe using them we can solve these six unknown $D$ coefficients; thus, we find the BCJ expansions (2.13) as the consistent conditions for differential operators.

\section{CONCLUSION AND DISCUSSION}

The double copy and the related KLT relation imply that amplitudes of one theory can be expanded in terms of the amplitudes of another theory as in (1.5). Although this is, in principle, a simple fact, directly using the KLT form (1.4) to find expansion coefficients is a very nontrivial task despite many efforts devoted to finding alternative ways.

In this paper, we have proposed a new method to efficiently find expansion coefficients by using the differential operators introduced in [34]. Moreover, we have actually achieved more than our initial goal. Using the proper building blocks, we have shown that the differential operators, together with the web connections established in [34], naturally lead to the expansion of the sEYM amplitude in the KK basis or BCJ basis without any extra assumptions. Furthermore, with the use of these building blocks, we have translated the problem into a set of linear equations, thus greatly reducing its complexity.

For the expansion in the KK basis, the expansion coefficients are polynomial and the natural building blocks are $\epsilon \cdot \epsilon$ and $\epsilon \cdot k$. With this simplification, the recursive expansion of sEYM amplitudes to YM amplitudes (2.22) has been reproduced by the new method. As a further demonstration of the efficacy of the new techniques, we have also discussed the expansion of gravity theory and Born-Infeld theory in the KK basis.

For the expansion in the $\mathrm{BCJ}$ basis, which has not been dealt with much in previous works, finding the expansion coefficients is a much more difficult task. The technical challenge lies in that a proper understanding of the building blocks, for arbitrary number of gravitons, is still missing. As explained above, the building blocks $\epsilon \cdot \epsilon$ and $\epsilon \cdot k$ in the KK-basis expansion are too large. Even if we constrain ourselves to the gauge invariant combinations, such as $\left(k_{1} f X\right)$ and $(k f \ldots f k)$, they are still too large. As we have argued, the index cycle structure should not appear. Furthermore, as given in (5.40), not all allowed gauge invariant building blocks are independent of each other. Thus, a proper understanding of these building blocks becomes one of the most important problems.

Both expansions are worth studying. For the expansion in the KK basis, since all physical poles are included in the basis, expansion coefficients can be arranged to be polynomials. Thus, such an expansion is more suitable when considering the analytical structure of amplitudes. However, in this manner, the manifest gauge invariance of gravitons is lost. On the other hand, for the expansion in the BCJ basis, the gauge invariance of all gravitons is manifest. The price to pay is that some physical poles are moved to the coefficients.

Because of the difficulty related to gauge invariant building blocks, as pointed out before, we could not give the complete solution for the expansion of sEYM amplitudes in the BCJ basis of YM amplitudes at this moment. Thus, a clear understanding of the building blocks will be an important future problem. Our examples of the previous section, especially the recursive construction, may provide us with some guidance in this issue. Our results here show that differential operators introduced in [34] provide a much wider set of applications [such as the three generalized relations presented in (2.31), (2.37), and (2.38)] and have a deeper meaning [such as the relation between expansion coefficients and BCJ expansion coefficients given in (6.44)] than previously thought. Therefore, further studies are needed in order to exploit their full potential, such as, for instance, their applications to soft and collinear limits.

\section{ACKNOWLEDGMENTS}

This work is supported by Qiu-Shi Funding and Chinese Natural Science Foundation (NSF) funding under Grants No. 11575156, No. 11935013, and No. 11805163, as well as NSF funding of Jiangsu Province under Grant No. BK20180897.

\section{APPENDIX A: TERMS WITH INDEX CIRCLE STRUCTURE}

When we try to derive the recursive expansion of sEYM amplitudes, we encounter some terms like $\left(\epsilon_{h_{1}} \cdot f_{h_{j}} \cdot f_{h_{j_{2}}} \cdot k_{h_{1}}\right) B_{h_{j} h_{j_{2}} h_{1}}$ and $\left(\epsilon_{h_{1}} \cdot f_{h_{j}} \cdot f_{h_{j_{2}}} \cdot k_{h_{j}}\right) B_{h_{j} h_{j_{2}} h_{j}}$, where $f=k \epsilon-\epsilon k$. If we expand $f$ in these corresponding building blocks completely, such as $\left(\epsilon_{h_{1}} \cdot f_{h_{j}} \cdot f_{h_{j_{2}}} \cdot k_{h_{1}}\right)$ and $\left(\epsilon_{h_{1}} \cdot f_{h_{j}} \cdot f_{h_{j_{2}}} \cdot k_{h_{j}}\right)$, we will get terms containing factors like $\left(\epsilon_{i_{1}} \cdot k_{i_{2}}\right)\left(\epsilon_{i_{2}} \cdot k_{i_{3}}\right) \cdots\left(\epsilon_{i_{s}} \cdot k_{i_{1}}\right)$, where all $i_{t}$ are gravitons. We will call such a structure the "index cycle structure." In this appendix we will show that all building blocks with the index cycle structure will not appear in the expansion.

To prove the above claim, we need to consider some special combinations of insertion operators, such as the $\mathcal{T}_{a h_{i_{1}} h_{i_{2}}} \mathcal{T}_{a h_{i_{2}} h_{i_{3}}} \cdots \mathcal{T}_{a h_{i_{s}} h_{i_{1}}}$, where $a$ represents a gluon and $h_{i}$ 's represent gravitons. Acting on both sides of the expansion of the sEYM amplitude $A_{n, m}^{\mathrm{EYM}}$, on the right-hand side, only the $\left(\epsilon_{h_{i_{1}}} f_{h_{i_{2}}} \cdots f_{h_{i_{s}}} k_{h_{i_{1}}}\right) B_{h_{i_{1}} \cdots h_{i_{s}} h_{i_{1}}}$ term is not annihilated, while at the left-hand side we have 


$$
\mathcal{T}_{a h_{i_{1}} h_{i_{2}}} \mathcal{T}_{a h_{i_{2}} h_{i_{3}}} \cdots \mathcal{T}_{a h_{i_{s}} h_{i_{1}}} A_{n, m}^{\mathrm{EYM}}=\int d \mu \mathrm{PT}(1,2, \ldots, n)\left(\mathcal{T}_{a h_{i_{1}} h_{i_{2}}} \mathcal{T}_{a h_{i_{2}} h_{i_{3}}} \cdots \mathcal{T}_{a h_{i_{s}} h_{i_{1}}} \mathrm{Pf}_{H_{m}}\right) \mathrm{Pf}^{\prime} \Psi
$$

Thus, to fix unknown $B_{h_{i_{1}} \cdots h_{i_{s}} h_{i_{1}}}$ we just need to work out the effect of these operators on $\operatorname{Pf} \Psi_{H_{m}}$.

According to $[43,44], \mathrm{Pf} \Psi_{H_{m}}$ can be expanded as the sum of permutations like

$$
\operatorname{Pf} \Psi_{H_{m}}=\sum_{\substack{1 \leq i_{1} \leq i_{2} \leq \cdots i_{m} \leq n \\ i_{1}+i_{2}+\cdots+i_{m}=n}}(-1)^{n-m} P_{i_{1} i_{2} \cdots i_{m}}
$$

where the sum is organized by the unique cycle decomposition of these permutations. When the length of a cycle is one,

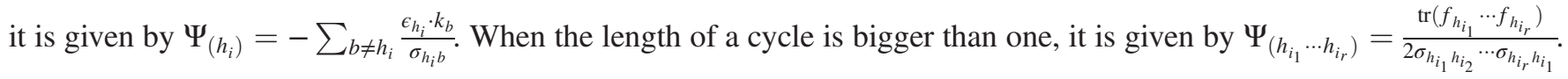
For example,

$$
\operatorname{Pf} \Psi_{3}=P_{111}-P_{12}+P_{3}=\Psi_{(1)} \Psi_{(2)} \Psi_{(3)}-\Psi_{(1)} \Psi_{(23)}-\Psi_{(2)} \Psi_{(13)}-\Psi_{(3)} \Psi_{(12)}+\Psi_{(123)}+\Psi_{(132)}
$$

Having the expression of $\mathrm{Pf}_{H_{m}}$, we present two simple examples to show why (A1) is zero at the first, and then a rigorous proof follows. Let us start with the simplest case, i.e., the one with only two gravitons. Without loss of generality, let us consider the operator $\mathcal{T}_{a h_{1} h_{2}} \mathcal{T}_{a h_{2} h_{1}}$. Putting it to (A1), we have

$$
\begin{aligned}
\mathcal{T}_{a h_{1} h_{2}} \mathcal{T}_{a h_{2} h_{1}} \operatorname{Pf}_{H_{m}} & =\mathcal{T}_{a h_{1} h_{2}} \mathcal{T}_{a h_{2} h_{1}} \sum_{\substack{1 \leq i_{1} \leq i_{2} \leq \cdots \leq i_{m} \leq n \\
i_{1}+i_{2}+\cdots+i_{m}=n}}(-1)^{n-m} P_{i_{1} i_{2} \cdots i_{m}} \\
& =\mathcal{T}_{a h_{1} h_{2}} \mathcal{T}_{a h_{2} h_{1}}\left\{\Psi_{(1)} \Psi_{(2)} \Psi_{H_{m-2}}-\Psi_{(12)} \Psi_{H_{m-2}}+\Psi_{(1)} \Psi_{(2 \cdots)}(\cdots)+\Psi_{(2)} \Psi_{(1 \cdots)}(\cdots)+\Psi_{(1 \ldots 2 \ldots)}(\cdots)\right\} \\
& =\mathcal{T}_{a h_{1} h_{2}} \mathcal{T}_{a h_{2} h_{1}}\left\{\Psi_{(1)} \Psi_{(2)}-\Psi_{(12)}\right\} \operatorname{Pf} \Psi_{H_{m-2}} .
\end{aligned}
$$

In (A4), $\Psi_{(2 \cdots)}$ represents any cycle containing $h_{2}$ and gravitons other than $h_{1}$, and a similar understanding for $\Psi_{(1 \cdots)}$ and $\Psi_{(1 \ldots 2 \ldots)}$. All of them are annihilated by at least one of the insertion operators. Thus, among all terms in the sum of Pf $\Psi$, only the first two give nonzero contributions. Carrying it out explicitly, we get

$\mathcal{T}_{a h_{1} h_{2}} \mathcal{T}_{a h_{2} h_{1}} \operatorname{Pf}_{H_{m}}=\mathcal{T}_{a h_{1} h_{2}} \mathcal{T}_{a h_{2} h_{1}}\left\{\Psi_{(1)} \Psi_{(2)}-\Psi_{(12)}\right\} \operatorname{Pf} \Psi_{H_{m-2}}=\left\{\frac{\sigma_{h_{2} a}}{\sigma_{h_{1} a} \sigma_{h_{1} h_{2}}} \frac{\sigma_{h_{1} a}}{\sigma_{h_{2} h_{1}} \sigma_{h_{2} a}}-\frac{1}{\sigma_{h_{1} h_{2}} \sigma_{h_{2} h_{1}}}\right\} \operatorname{Pf} \Psi_{H_{m-2}}=0$

The next simple case is the one with three gravitons, for example, the string $\left(h_{1}, h_{2}, h_{3}\right)$. By a similar reason, the action of $\mathcal{T}_{a h_{1} h_{2}} \mathcal{T}_{a h_{2} h_{3}} \mathcal{T}_{a h_{3} h_{1}}$ is

$$
\begin{aligned}
\mathcal{T}_{a h_{1} h_{2}} \mathcal{T}_{a h_{2} h_{3}} \mathcal{T}_{a h_{3} h_{1}} \mathrm{Pf}_{H_{m}}= & \mathcal{T}_{a h_{1} h_{2}} \mathcal{T}_{a h_{2} h_{3}} \mathcal{T}_{a h_{3} h_{1}}\left\{\Psi_{\left(h_{1}\right)} \Psi_{\left(h_{2}\right)} \Psi_{\left(h_{3}\right)}-\Psi_{\left(h_{1}\right)} \Psi_{\left(h_{2} h_{3}\right)}-\Psi_{\left(h_{2}\right)} \Psi_{\left(h_{1} h_{3}\right)}-\Psi_{\left(h_{3}\right)} \Psi_{\left(h_{1} h_{2}\right)}\right. \\
& \left.+\Psi_{\left(h_{1} h_{2} h_{3}\right)}+\Psi_{\left(h_{1} h_{3} h_{2}\right)}\right\} \operatorname{Pf} \Psi_{H_{m-3}},
\end{aligned}
$$

with $\operatorname{Pf} \Psi_{H_{m-2}}$ the Pfaffian of other gravitons. Carrying it out explicitly, first we note that

$$
\mathcal{T}_{a h_{1} h_{2}} \Psi_{\left(h_{1}\right)} \mathcal{T}_{a h_{2} h_{3}} \mathcal{T}_{a h_{3} h_{1}} \Psi_{\left(h_{2} h_{3}\right)}=\frac{\sigma_{h_{2} a}}{\sigma_{h_{1} h_{2}} \sigma_{h_{1} a}} \mathcal{T}_{a h_{2} h_{3}}\left\{\mathcal{T}_{a h_{3} h_{1}} \frac{\left[\left(\epsilon_{h_{2}} f_{h_{3}} k_{h_{2}}\right)-\left(k_{h_{2}} f_{h_{3}} \epsilon_{h_{2}}\right)\right]}{2 \sigma_{h_{2} h_{3}} \sigma_{h_{3} h_{2}}}\right\}=0,
$$

because $\Psi_{\left(h_{2} h_{3}\right)}$ does not contain $\left(\epsilon_{h_{3}} k_{h_{1}}\right)$ and $\left(\epsilon_{h_{3}} k_{a}\right)$; thus, $\mathcal{T}_{a h_{3} h_{1}} \Psi_{\left(h_{2} h_{3}\right)}=0$. Similarly, we have

$$
\mathcal{T}_{a h_{2} h_{3}} \Psi_{\left(h_{2}\right)} \mathcal{T}_{a h_{1} h_{2}} \mathcal{T}_{a h_{3} h_{1}} \Psi_{\left(h_{1} h_{3}\right)}=0, \quad \mathcal{T}_{a h_{3} h_{1}} \Psi_{\left(h_{3}\right)} \mathcal{T}_{a h_{1} h_{2}} \mathcal{T}_{a h_{2} h_{3}} \Psi_{\left(h_{1} h_{2}\right)}=0
$$

For the remaining three terms, we have 


$$
\begin{aligned}
\mathcal{T}_{a h_{1} h_{2}} \mathcal{T}_{a h_{2} h_{3}} \mathcal{T}_{a h_{3} h_{1}} \mathrm{Pf}_{H_{m}} & =\left\{\frac{\sigma_{h_{2} a}}{\sigma_{h_{1} h_{2}} \sigma_{h_{1} a}} \frac{\sigma_{h_{3} a}}{\sigma_{h_{2} h_{3}} \sigma_{h_{2} a}} \frac{\sigma_{h_{1} a}}{\sigma_{h_{3} h_{1}} \sigma_{h_{3} a}}-\frac{1}{2 \sigma_{h_{1} h_{2}} \sigma_{h_{2} h_{3}} \sigma_{h_{3} h_{1}}}+\frac{1}{2 \sigma_{h_{1} h_{3}} \sigma_{h_{3} h_{2}} \sigma_{h_{2} h_{1}}}\right\} \operatorname{Pf} \Psi_{H_{m-3}} \\
& =\left\{\frac{1}{\sigma_{h_{1} h_{2}} \sigma_{h_{2} h_{3}} \sigma_{h_{3} h_{1}}}-\frac{1}{2 \sigma_{h_{1} h_{2}} \sigma_{h_{2} h_{3}} \sigma_{h_{3} h_{1}}}+\frac{1}{2 \sigma_{h_{1} h_{3}} \sigma_{h_{3} h_{2}} \sigma_{h_{2} h_{1}}}\right\} \mathrm{Pf}_{H_{m-3}}=0 .
\end{aligned}
$$

From the previous two simple examples, we can see how to generalize to strings of $s$ gravitons, for example, the $\left(h_{1}, h_{2}, \ldots, h_{s}\right)$. With the action of insertion operators, we have

$$
\begin{aligned}
\mathcal{T}_{a h_{1} h_{2}} \mathcal{T}_{a h_{2} h_{3}} \cdots \mathcal{T}_{a h_{s} h_{1}} \operatorname{Pf}_{H_{m}} & =\mathcal{T}_{a h_{1} h_{2}} \mathcal{T}_{a h_{2} h_{3}} \cdots \mathcal{T}_{a h_{s} h_{1}} \sum_{\substack{1 \leq i_{1} \leq i_{2} \leq \cdots \leq i_{m} \leq n \\
i_{1}+i_{2}+\cdots+i_{m}=n}}(-1)^{n-m} P_{i_{1} i_{2} \cdots i_{m}} \\
& =\mathcal{T}_{a h_{1} h_{2}} \mathcal{T}_{a h_{2} h_{3}} \cdots \mathcal{T}_{a h_{s} h_{1}}\left\{\sum_{\substack{1 \leq j_{1} \leq j_{2} \leq \cdots \leq j_{l} \leq s \\
j_{1}+j_{2}+\cdots+j_{l}=s}}(-1)^{s-1} P_{j_{1} j_{2} \cdots j_{l}}\right\} \operatorname{Pf} \Psi_{H_{m-s}},
\end{aligned}
$$

where $\operatorname{Pf} \Psi_{H_{m-s}}$ is the Pfaffian of other gravitons. Because of the particular circle order $\left(h_{1}, h_{2}, \ldots, h_{s}\right)$, the action of insertion operator $\mathcal{T}_{a h_{j} h_{j+1}}$ is not zero when and only when there is the factor $\epsilon_{h_{j}} \cdot k_{a}$ or $\epsilon_{h_{j}} \cdot k_{h_{j+1}}$. Thus, only terms, which are either the multiplication of $s$ 's length one cycles or the length $s$ cycles having the same or reversing order, will contribute. With the simplification, we have

$$
\begin{aligned}
\mathcal{T}_{a h_{1} h_{2}} \mathcal{T}_{a h_{2} h_{3}} \cdots \mathcal{T}_{a h_{s} h_{1}} \operatorname{Pf}_{H_{m}}= & \mathcal{T}_{a h_{1} h_{2}} \mathcal{T}_{a h_{2} h_{3}} \cdots \mathcal{T}_{a h_{s} h_{1}}\left\{\Psi_{h_{1}} \Psi_{h_{2}} \cdots \Psi_{h_{s}}+(-1)^{s-1} \Psi_{\left(h_{1} h_{2} \cdots h_{s}\right)}+(-1)^{s-1} \Psi_{\left(h_{s} h_{s-1} \cdots h_{1}\right)}\right\} \Psi_{H_{m-s}} \\
= & \left\{\frac{\sigma_{h_{2} a}}{\sigma_{h_{1} a} \sigma_{h_{1} h_{2}}} \frac{\sigma_{h_{3} a}}{\sigma_{h_{2} a} \sigma_{h_{2} h_{3}}} \cdots \frac{\sigma_{h_{1} a}}{\sigma_{h_{s} a} \sigma_{h_{s} h_{1}}}+(-1)^{s-1}(-1)^{s} \frac{1}{2 \sigma_{h_{1} h_{2}} \sigma_{h_{2} h_{3}} \cdots \sigma_{h_{s} h_{1}}}\right. \\
& \left.+(-1)^{s-1} \frac{1}{2 \sigma_{h_{s} h_{s-1}} \sigma_{h_{s-1} h_{s-2}} \cdots \sigma_{h_{1} h_{s}}}\right\} \Psi_{H_{m-s}} \\
= & \left\{\frac{1}{\sigma_{h_{1} h_{2}} \sigma_{h_{2} h_{3}} \cdots \sigma_{h_{s} h_{1}}}-\frac{1}{\sigma_{h_{1} h_{2}} \sigma_{h_{2} h_{3}} \cdots \sigma_{h_{s} h_{1}}}\right\} \Psi_{H_{m-s}} \\
= & 0 .
\end{aligned}
$$

So finally we have proved that any term with the building block having an index circle structure will vanish.

\section{APPENDIX B: GENERAL DISCUSSION OF MANIFESTLY GAUGE INVARIANT FUNCTIONS}

In this appendix, we make some general discussions of manifestly gauge invariant functions, which are important for the construction of building blocks when expanding in the BCJ basis. For amplitudes involving gauge particles, they must satisfy some basic requirements: Lorentz invariance, on-shell condition, momentum conservation, transversality, ${ }^{19}$ gauge invariance, etc. With those requirements, properties of amplitudes have been thoroughly discussed in [49-53]. Especially, in [51], starting from the fact that amplitudes are multilinear functions of $\epsilon$ 's, the authors are able to write down a set of linear equations coming from gauge invariance condition. After solving them, they can derive some important consequence. Going further in [52] the authors prove that using only gauge invariance, locality,

\footnotetext{
${ }^{19}$ Here, we follow the name from [49]; it means $\epsilon_{i} \cdot k_{i}=0$.
}

and minimal power counting, one can determine amplitudes uniquely.

Since gauge invariance is so important and a strong constraint, it is better to construct functions which are manifestly gauge invariant by sacrificing other properties [53]. By Feynman rules, amplitudes are multilinear functions of polarization vectors. The gauge invariant condition means that when replacing $\epsilon_{i}^{\mu}$ by $k_{i}^{\mu}$ for each $i$, amplitudes vanish. A simple and naive combination satisfying the above condition is $\left(\epsilon_{i}^{\mu}-k_{i}^{\mu}\right)$, which is wrong since the dimension of two terms does not match. Correcting with the dimension, one leads to the form $\left(\epsilon_{i}^{\mu} k_{j}^{\nu}-\epsilon_{r}^{\nu} k_{i}^{\mu}\right)$. Imposing the gauge invariant condition for each $i$, we end up with the combination $\epsilon_{i}^{\mu} k_{i}^{\nu}-k_{i}^{\mu} \epsilon_{i}^{\nu}$, which is nothing, but the familiar field strength $f_{i}^{\mu \nu}=k_{i}^{\mu} \epsilon_{i}^{\nu}-\epsilon_{i}^{\mu} k_{i}^{\nu}$. The above argument seems to indicate that any manifestly gauge invariant function could be rewritten as the function of $f^{\mu \nu}$. Although we could not give complete proof about this statement, for some 
special cases, such as the sEYM amplitudes studied in this paper, we will show it now.

Actually, we can go further. Lorentz invariance requires that $f^{\mu \nu}$ must be contracted by a momentum or another $f^{\mu \nu}$; thus, eventually we will reach two types of contractions: Type I with the form $\left(k_{a} \cdot f_{b} \cdots f_{c} \cdot k_{d}\right)$ and type II with the form $\operatorname{tr}\left(f_{a} \cdot f_{b} \cdots f_{c} \cdot f_{d}\right) \equiv\left(f_{a \nu}^{\mu} f_{b \rho}^{\nu} \cdots f_{c \beta}^{\alpha} f_{d \mu}^{\beta}\right)$. Lorentz and gauge invariant building blocks should be functions of both types, but in this paper we only encounter the type-I contractions, ${ }^{20}$ so we just discuss some properties of them.

For the type-I contraction, we use the number of $f$ 's appearing in the term to characterize them (called the $f$ degree); thus, we have $d_{f}=m-2$, where $d_{f}$ is the $f$ degree while $m$ is the mass dimension. Some examples are $\left(k_{a} f_{b} k_{c}\right)$ of degree one and $\left(k_{i} f_{j} f_{l} k_{s}\right)$ of degree two. Two properties of type-I contractions can be easily proved. The first is

$$
\left(k_{i_{1}} f_{i_{2}} \cdots f_{i_{m-1}} k_{i_{m}}\right)=(-1)^{m-2}\left(k_{i_{m}} f_{i_{m-1}} \cdots f_{i_{2}} k_{i_{1}}\right) .
$$

The second is

$$
\begin{aligned}
& \left(k_{i_{1}} f_{i_{2}} \cdots f_{i_{a-1}} f_{i_{a}} f_{i_{a+1}} \cdots f_{i_{m-1}} k_{i_{m}}\right)\left(k_{j} k_{i_{a}}\right) \\
& =\left(k_{i_{1}} f_{i_{2}} \cdots f_{i_{a-1}} k_{i_{a}}\right)\left(k_{j} f_{i_{a}} f_{i_{a+1}} \cdots f_{i_{m-1}} k_{i_{m}}\right) \\
& \quad+\left(k_{i_{1}} f_{i_{2}} \cdots f_{i_{a-1}} f_{i_{a}} k_{j}\right)\left(k_{i_{a}} f_{i_{a+1}} \cdots f_{i_{m-1}} k_{i_{m}}\right),
\end{aligned}
$$

which is just the identity (5.14). The formula (B2) tells us that any type-I contraction with higher degrees can always be decomposed to the sum of terms as the product of type-I contractions with lower degrees. This decomposition terminates at type-I contractions of degree one and degree two, which will be called fundamental. Relation (B2) gives the first indication of why the building blocks for the BCJ basis are much more complicated.

Having the above general discussions, let us move to the case related to sEYM amplitudes.

\section{Having only one polarization vector}

Now we consider the simplest example, i.e., a general function of $(n+1)$ momenta and only one polarization vector. As the linear functions of $\epsilon$, the functions can be generally written as

$$
F=F\left(k_{1}, \ldots, k_{n}, k_{p}, \epsilon_{p}\right)=\sum_{i=1}^{n-1} \alpha_{i}\left(\epsilon_{p} \cdot k_{i}\right),
$$

where $\alpha_{i}$ 's are unknown functions of $\left(k_{j} \cdot k_{l}\right)$. Here we have used momentum conservation to eliminate the momentum $k_{n}$, so all remaining $\left(\epsilon_{p} \cdot k_{i}\right)$ 's are independent. The gauge invariant condition leads to

\footnotetext{
${ }^{20}$ In [53], the four-point amplitude of gauge theory has been rewritten to a form using the type-II contractions.
}

$$
F\left(\epsilon_{p} \rightarrow k_{p}\right)=\sum_{i=1}^{n-1} \alpha_{i}\left(k_{p} \cdot k_{i}\right)=0
$$

Solving

$$
\alpha_{1}=-\sum_{i=2}^{n-1} \alpha_{i} \frac{\left(k_{p} \cdot k_{i}\right)}{\left(k_{p} \cdot k_{1}\right)},
$$

from the equation and putting it back, we get

$$
\begin{aligned}
F & =\sum_{i=2}^{n-1} \alpha_{i}\left(\epsilon_{p} \cdot k_{i}\right)-\left(\epsilon_{p} \cdot k_{1}\right)\left[-\sum_{i=2}^{n-1} \alpha_{i} \frac{\left(k_{p} \cdot k_{i}\right)}{\left(k_{p} \cdot k_{1}\right)}\right] \\
& =\sum_{i=2}^{n-1} \alpha_{i} \frac{\left(k_{1} f_{p} k_{i}\right)}{\mathcal{K}_{1 p}}
\end{aligned}
$$

with $\left(k_{1} f_{p} k_{i}\right)=k_{1 \mu}\left(k_{p}^{\mu} \epsilon_{p}^{\nu}-\epsilon_{p}^{\mu} k_{p}^{\nu}\right) k_{i \nu}$ and $\mathcal{K}_{1 p}=\left(k_{p} \cdot k_{1}\right)$. Thus, we have shown the appearance of type-I contractions in this case. ${ }^{21}$ Result (B6) tells us that the basis of gauge invariant building blocks of the single polarization vector is given by $\left(k_{1} f_{p} k_{i}\right)$ (or $\left.\frac{\left(k_{1} f_{p} k_{i}\right)}{\mathcal{K}_{1 p}}\right)$ with $i=2, \ldots, n-1$. An equivalent, but more convenient basis, can be taken as $\left(k_{1} f_{p} K_{i}\right) \quad\left(\right.$ or $\left.\frac{\left(k_{1} f_{p} K_{i}\right)}{\mathcal{K}_{1 p}}\right)$ with $i=2, \ldots, n-1$ and $K_{i}=\sum_{t=1}^{i} k_{t}$.

\section{Having two polarization vectors}

Now we consider the case with $(n+2)$ momenta and two polarization vectors. The general form of the function $F$ is

$$
\begin{aligned}
F & =F\left(k_{1}, \ldots, k_{n}, k_{p}, k_{q}, \epsilon_{p}, \epsilon_{q}\right) \\
& =\alpha\left(\epsilon_{p} \cdot \epsilon_{q}\right)+\sum_{i=1}^{n-1, q} \sum_{j=1}^{n-1, p} \beta_{i j}\left(\epsilon_{p} \cdot k_{i}\right)\left(\epsilon_{q} \cdot k_{j}\right),
\end{aligned}
$$

where we have used the momentum conservation to eliminate the contractions $\left(\epsilon_{p} \cdot k_{n}\right)$ and $\left(\epsilon_{q} \cdot k_{n}\right)$. The gauge invariant condition of $p$ leads to

$$
\begin{aligned}
F\left(\epsilon_{p} \rightarrow k_{p}\right)= & \alpha\left(k_{p} \cdot \epsilon_{q}\right)+\sum_{i=1}^{n-1, q} \sum_{j=1}^{n-1, p} \beta_{i j}\left(k_{p} \cdot k_{i}\right)\left(\epsilon_{q} \cdot k_{j}\right) \\
= & \left(\epsilon_{q} \cdot k_{p}\right)\left[\alpha+\sum_{i=1}^{n-1, q} \beta_{i p}\left(k_{p} \cdot k_{i}\right)\right] \\
& +\sum_{j=1}^{n-1}\left[\sum_{i=1}^{n-1, q} \beta_{i j}\left(k_{p} \cdot k_{i}\right)\right]\left(\epsilon_{q} \cdot k_{j}\right) \\
= & 0 .
\end{aligned}
$$

\footnotetext{
${ }^{21}$ We should note that the number of momenta should be greater than 3 , since when $n+1=3$, the function will vanish because of the special kinematics of three particles.
} 
By the independence of $\left(\epsilon_{q} \cdot k_{p}\right)$ and $\left(\epsilon_{q} \cdot k_{j}\right)$ 's, we get the first set of equations

$$
\left\{\begin{array}{l}
\alpha+\sum_{i=1}^{n-1, q} \beta_{i p}\left(k_{p} \cdot k_{i}\right)=0 \\
\sum_{i=1}^{n-1, q} \beta_{i j}\left(k_{p} \cdot k_{i}\right)=0
\end{array} \text { for } j=1,2, \ldots, n-1 .\right.
$$

Similarly the gauge invariant condition of $q$ gives us another set of equations

$$
\left\{\begin{array}{l}
\alpha+\sum_{j=1}^{n-1, p} \beta_{q j}\left(k_{q} \cdot k_{j}\right)=0 \\
\sum_{j=1}^{n-1, p} \beta_{i j}\left(k_{q} \cdot k_{j}\right)=0
\end{array} \quad \text { for } i=1,2, \ldots, n-1 .\right.
$$

Now we solve these equations. Using the first set of equations we get

$$
\begin{aligned}
& \beta_{1 p}=-\alpha \frac{1}{\left(k_{p} \cdot k_{1}\right)}-\sum_{i=2}^{n-1, q} \beta_{i p} \frac{\left(k_{p} \cdot k_{i}\right)}{\left(k_{p} \cdot k_{1}\right)} \\
& \beta_{1 j}=-\sum_{i=2}^{n-1, q} \beta_{i j} \frac{\left(k_{p} \cdot k_{i}\right)}{\left(k_{p} \cdot k_{1}\right)} \quad \text { for } j=1,2, \ldots, n-1,
\end{aligned}
$$

while using the second set of equations we get

$$
\begin{aligned}
& \beta_{q 1}=-\alpha \frac{1}{\left(k_{1} \cdot k_{q}\right)}-\sum_{j=2}^{n-1, p} \beta_{q j} \frac{\left(k_{q} \cdot k_{j}\right)}{\left(k_{1} \cdot k_{q}\right)} \\
& \beta_{i 1}=-\sum_{j=2}^{n-1, p} \beta_{i j} \frac{\left(k_{q} \cdot k_{j}\right)}{\left(k_{1} \cdot k_{q}\right)} \quad \text { for } i=1,2, \ldots, n-1 .
\end{aligned}
$$

Putting these results back and doing some algebraic manipulations, we reach

$$
F=-\alpha \frac{\left(k_{1} \cdot f_{p} \cdot f_{q} \cdot k_{1}\right)}{\left(k_{1} \cdot k_{p}\right)\left(k_{1} \cdot k_{q}\right)}+\sum_{i=2}^{n-1, q} \sum_{j=2}^{n-1, p} \beta_{i j} \frac{\left(k_{1} \cdot f_{p} \cdot k_{i}\right)}{\left(k_{1} \cdot k_{p}\right)} \frac{\left(k_{1} \cdot f_{q} \cdot k_{j}\right)}{\left(k_{1} \cdot k_{q}\right)}
$$

which is manifestly gauge invariant with only the field strength appearing at the cost of introducing poles $\left(k_{1} \cdot k_{p}\right)$ and $\left(k_{1} \cdot k_{q}\right){ }^{22}$ The above result is applicable when $(n+2)>4$. For the special case $n=2$, the $F$ is

$$
F=-\alpha \frac{\left(k_{1} \cdot f_{p} \cdot f_{q} \cdot k_{1}\right)}{\left(k_{1} \cdot k_{p}\right)\left(k_{1} \cdot k_{q}\right)}+\beta_{q p} \frac{\left(k_{1} \cdot f_{p} \cdot k_{q}\right)}{\left(k_{1} \cdot k_{p}\right)} \frac{\left(k_{1} \cdot f_{q} \cdot k_{p}\right)}{\left(k_{1} \cdot k_{q}\right)} .
$$

Result (B11) shows that with two polarization vectors, the basis of gauge invariant building blocks contains the following three types:

$$
\begin{array}{ll}
\text { type-I: } & \frac{\left(k_{1} \cdot f_{p} \cdot k_{i}\right)}{\left(k_{1} \cdot k_{p}\right)} \frac{\left(k_{1} \cdot f_{q} \cdot k_{j}\right)}{\left(k_{1} \cdot k_{q}\right)}, \quad i, j=2, \ldots, n-2 \\
\text { type-II: } & \frac{\left(k_{1} \cdot f_{p} \cdot k_{q}\right)}{\left(k_{1} \cdot k_{p}\right)} \frac{\left(k_{1} \cdot f_{q} \cdot k_{j}\right)}{\left(k_{1} \cdot k_{q}\right)}, \\
\text { type-III: } & \frac{\left(k_{1} \cdot f_{p} \cdot f_{q} \cdot k_{1}\right)}{\left(k_{1} \cdot k_{p}\right)\left(k_{1} \cdot k_{q}\right)}
\end{array}
$$

We can also replace $k_{i} \rightarrow K_{i}$ to get the equivalent basis.

\section{Having three polarization vectors}

Now we consider the case with three polarization vectors. The general form of $F$ can be written as

$$
F=\alpha^{i j l}\left(\epsilon_{n+1} \cdot k_{i}\right)\left(\epsilon_{n+2} \cdot k_{j}\right)\left(\epsilon_{n+3} \cdot k_{l}\right)+\beta_{1}^{i}\left(\epsilon_{n+1} \cdot k_{i}\right)\left(\epsilon_{n+2} \cdot \epsilon_{n+3}\right)+\beta_{2}^{j}\left(\epsilon_{n+2} \cdot k_{j}\right)\left(\epsilon_{n+1} \cdot \epsilon_{n+3}\right)+\beta_{3}^{l}\left(\epsilon_{n+3} \cdot k_{l}\right)\left(\epsilon_{n+1} \cdot \epsilon_{n+2}\right)
$$

where the summations of $i, j, l$ are implicit for simplicity, and their ranges are $i \in\{1,2, \ldots, n-1, n+2, n+3\}$, $j \in\{1,2, \ldots, n-1, n+1, n+3\}$, and $l \in\{1,2, \ldots, n-1, n+1, n+2\}$. Again we have used the momentum conservation to eliminate $k_{n}$.

\footnotetext{
${ }^{22}$ There is a choice when solving $\beta$ 's. Different choices lead to different poles.
} 
Using the gauge invariant conditions for $\epsilon_{n+1}, \epsilon_{n+2}$, and $\epsilon_{n+3}$, we can get three sets of linear equations. The first set is

$$
\begin{aligned}
& \sum_{i \neq n, n+1} \beta_{1}^{i}\left(k_{n+1} \cdot k_{i}\right)=0, \\
& \sum_{i \neq n, n+1} \alpha^{i(n+1)(n+1)}\left(k_{n+1} \cdot k_{i}\right)+\beta_{2}^{n+1}+\beta_{3}^{n+1}=0, \\
& \sum_{i \neq n, n+1} \alpha^{i j(n+1)}\left(k_{n+1} \cdot k_{i}\right)+\beta_{2}^{j}=0, \quad \text { for } j \neq n, n+1, n+2, \\
& \sum_{i \neq n, n+1} \alpha^{i(n+1) l}\left(k_{n+1} \cdot k_{i}\right)+\beta_{3}^{l}=0, \quad \text { for } l \neq n, n+1, n+3, \\
& \sum_{i \neq n, n+1} \alpha^{i j l}\left(k_{n+1} \cdot k_{i}\right)=0, \quad \text { for } j \neq n, n+1, n+2 \text { and } l \neq n, n+1, n+3 .
\end{aligned}
$$

The second set is

$$
\begin{aligned}
& \sum_{j \neq n, n+2} \beta_{2}^{j}\left(k_{n+2} \cdot k_{j}\right)=0, \\
& \sum_{j \neq n, n+2} \alpha^{(n+2) j(n+2)}\left(k_{n+2} \cdot k_{j}\right)+\beta_{1}^{n+2}+\beta_{3}^{n+2}=0, \\
& \sum_{j \neq n, n+2} \alpha^{i j(n+2)}\left(k_{n+2} \cdot k_{j}\right)+\beta_{1}^{i}=0, \quad \text { for } i \neq n, n+1, n+2, \\
& \sum_{j \neq n, n+2} \alpha^{(n+2) j l}\left(k_{n+2} \cdot k_{j}\right)+\beta_{3}^{l}=0, \quad \text { for } l \neq n, n+2, n+3, \\
& \sum_{j \neq n, n+2} \alpha^{i j l}\left(k_{n+2} \cdot k_{j}\right)=0, \quad \text { for } i \neq n, n+1, n+2 \quad \text { and } \quad l \neq n, n+2, n+3 .
\end{aligned}
$$

The third set is

$$
\begin{aligned}
& \sum_{l \neq n, n+3} \beta_{3}^{l}\left(k_{n+3} \cdot k_{l}\right)=0, \\
& \sum_{l \neq n, n+3} \alpha^{(n+3)(n+3) l}\left(k_{n+3} \cdot k_{l}\right)+\beta_{1}^{n+3}+\beta_{2}^{n+3}=0, \\
& \sum_{l \neq n, n+3} \alpha^{i(n+3) l}\left(k_{n+3} \cdot k_{l}\right)+\beta_{1}^{i}=0, \quad \text { for } i \neq n, n+1, n+3, \\
& \sum_{l \neq n, n+3} \alpha^{(n+3) j l}\left(k_{n+3} \cdot k_{l}\right)+\beta_{2}^{j}=0, \quad \text { for } j \neq n, n+2, n+3, \\
& \sum_{l \neq n, n+3} \alpha^{i j l}\left(k_{n+3} \cdot k_{l}\right)=0, \quad \text { for } i \neq n, n+1, n+3 \text { and } j \neq n, n+2, n+3 .
\end{aligned}
$$

For simplicity, in the sum we just indicate the unallowed indexes, for example, $i \neq n, n+1$ means the sum range is $i=1,2, \ldots, n-1, n+2, n+3$. From these three sets of equations we can solve some $\beta$ variables and then put them back to get the manifestly gauge invariant form of $F$ as

$$
\begin{aligned}
F= & \sum_{i \neq 1, n, n+1} \sum_{j \neq 1, n, n+2} \sum_{l \neq 1, n, n+3} \alpha^{i j l} \frac{\left(k_{1} \cdot f_{n+1} \cdot k_{i}\right)}{\left(k_{1} \cdot k_{n+1}\right)} \frac{\left(k_{1} \cdot f_{n+2} \cdot k_{j}\right)}{\left(k_{1} \cdot k_{n+2}\right)} \frac{\left(k_{1} \cdot f_{n+3} \cdot k_{l}\right)}{\left(k_{1} \cdot k_{n+3}\right)}-\sum_{i \neq 1, n, n+1} \beta_{1}^{i} \frac{\left(k_{1} \cdot f_{n+1} \cdot k_{i}\right)}{\left(k_{1} \cdot k_{n+1}\right)} \frac{\left(k_{1} \cdot f_{n+2} \cdot f_{n+3} \cdot k_{1}\right)}{\left(k_{1} \cdot k_{n+2}\right)\left(k_{1} \cdot k_{n+3}\right)} \\
& -\sum_{j \neq 1, n, n+2} \beta_{2}^{j} \frac{\left(k_{1} \cdot f_{n+2} \cdot k_{j}\right)}{\left(k_{1} \cdot k_{n+2}\right)} \frac{\left(k_{1} \cdot f_{n+1} \cdot f_{n+3} \cdot k_{1}\right)}{\left(k_{1} \cdot k_{n+1}\right)\left(k_{1} \cdot k_{n+3}\right)}-\sum_{l \neq 1, n, n+3} \beta_{3}^{l} \frac{\left(k_{1} \cdot f_{n+3} \cdot k_{l}\right)}{\left(k_{1} \cdot k_{n+3}\right)} \frac{\left(k_{1} \cdot f_{n+1} \cdot f_{n+1} \cdot k_{1}\right)}{\left(k_{1} \cdot k_{n+1}\right)\left(k_{1} \cdot k_{n+2}\right)} .
\end{aligned}
$$

As before, the special role of $k_{1}$ comes from our particular choice when solving equations. The above form is applicable when $n+3>4$. When $n+3=4, F$ is simplified to 


$$
F=\alpha^{i j l}\left(\epsilon_{2} \cdot k_{i}\right)\left(\epsilon_{3} \cdot k_{j}\right)\left(\epsilon_{4} \cdot k_{l}\right)+\beta_{1}^{i}\left(\epsilon_{2} \cdot k_{i}\right)\left(\epsilon_{3} \cdot \epsilon_{4}\right)+\beta_{2}^{j}\left(\epsilon_{3} \cdot k_{j}\right)\left(\epsilon_{2} \cdot \epsilon_{4}\right)+\beta_{3}^{l}\left(\epsilon_{4} \cdot k_{l}\right)\left(\epsilon_{2} \cdot \epsilon_{3}\right)
$$

with $i=3,4, j=2,4$, and $l=2,3$. Using gauge invariant conditions to get three sets of equations and solving them, we find

$$
\begin{aligned}
F= & \alpha^{342} \frac{\left(k_{4} \cdot f_{2} \cdot k_{3}\right)}{\left(k_{4} \cdot k_{2}\right)} \frac{\left(k_{2} \cdot f_{3} \cdot k_{4}\right)}{\left(k_{2} \cdot k_{3}\right)} \frac{\left(k_{3} \cdot f_{4} \cdot k_{2}\right)}{\left(k_{3} \cdot k_{4}\right)}-\beta_{1}^{3} \frac{\left(k_{4} \cdot f_{2} \cdot k_{3}\right)}{\left(k_{4} \cdot k_{2}\right)} \frac{\left(k_{2} \cdot f_{3} \cdot f_{4} \cdot k_{3}\right)}{\left(k_{2} \cdot k_{3}\right)\left(k_{3} \cdot k_{4}\right)} \\
& -\beta_{2}^{4} \frac{\left(k_{2} \cdot f_{3} \cdot k_{4}\right)}{\left(k_{2} \cdot k_{3}\right)} \frac{\left(k_{4} \cdot f_{2} \cdot f_{4} \cdot k_{3}\right)}{\left(k_{4} \cdot k_{2}\right)\left(k_{3} \cdot k_{4}\right)}-\beta_{3}^{2} \frac{\left(k_{3} \cdot f_{4} \cdot k_{2}\right)}{\left(k_{3} \cdot k_{4}\right)} \frac{\left(k_{4} \cdot f_{2} \cdot f_{3} \cdot k_{2}\right)}{\left(k_{4} \cdot k_{2}\right)\left(k_{3} \cdot k_{2}\right)} .
\end{aligned}
$$

Like the case in the previous subsection, result (B18) gives us also the basis of gauge invariant building blocks for three polarization vectors. Since it is straightforward, we will not write down them explicitly.

Although in this paper we will only use results up to three polarization vectors, the above calculations show that the manifestly gauge invariant form of $F$ does have some patterns, and we conjecture that these patterns will also appear even with more than three polarization vectors. But there must be some new things happen when the number of momenta is equal to that of polarization vectors. For example, when there are only four gluons involved, the manifestly gauge invariant form of $F$ has been given in [53] consisting of the type-II contractions, while the type-I contractions do not appear. We will discuss these in a future work.

\section{APPENDIX C: SOME CALCULATION DETAILS USING DIFFERENTIAL OPERATORS}

In this appendix, we present the explicit calculations for unknown variables $a\left(\rho^{\prime}, \rho\right)$ 's appearing in $C(\{p q r\}, ш)(6.46)$. Since coefficients are related by permutations, we could fix the ordering $\rho$ to be $\rho=\{p, q, r\}$ and use different orderings $\rho^{\prime}$ 's to denote different $a$ 's:

(1) $a(\{p, q, r\})$ : We have

$$
\begin{aligned}
a(\{p, q, r\})= & \mathcal{K}_{1 p}\left[\mathcal{C}^{(p q r)}(\{p, q, r\}, \omega)-\mathcal{T}_{1 p q r 2} C_{0}(\{p, q, r\}, ш)\right] \\
= & \mathcal{K}_{1 p}\left[\mathcal{C}^{(p q r)}(\{p, q, r\}, ш)-\mathcal{T}_{1 p 2} \frac{\left(k_{1} f_{p} X_{p}\right)}{\mathcal{K}_{1 p}} \mathcal{T}_{p q 2} \frac{\left(k_{1} f_{q} X_{q}\right)}{\mathcal{K}_{1 q}} \mathcal{T}_{q r 2} \frac{\left(k_{1} f_{r} X_{r}\right)}{\mathcal{K}_{1 r}}\right. \\
& \left.-\frac{\left[k_{p}\left(Y_{p}-k_{1}\right)\right]\left(k_{q} X_{q}\right)}{\mathcal{K}_{1 p q}} \mathcal{T}_{q r 2} \frac{\left(k_{1} f_{r} X_{r}\right)}{\mathcal{K}_{1 r}} \mathcal{T}_{p q 2} \mathcal{T}_{1 p 2} \frac{\left(k_{1} f_{q} f_{p} k_{1}\right)}{\mathcal{K}_{1 q} \mathcal{K}_{1 p}}\right] \\
= & \mathcal{K}_{1 p} \mathcal{C}^{(p q r)}(\{p, q, r\}, ш)=-\frac{\left[k_{p}\left(Y_{p}-k_{1}\right)\right]\left[\left(k_{q} X_{q}\right)-\mathcal{K}_{1 p q}\right]\left(k_{r} X_{r}\right)}{\mathcal{K}_{1 p q} \mathcal{K}_{1 p q r}} .
\end{aligned}
$$

(2) $a(\{p, r, q\}):$ We have

$$
\begin{aligned}
a(\{p, r, q\})= & \mathcal{K}_{1 p}\left[\mathcal{C}^{(p r q)}(\{p, q, r\}, ш)-\mathcal{T}_{1 p r q 2} C_{0}(\{p, q, r\}, ш)\right] \\
= & \mathcal{K}_{1 p}\left[\mathcal{C}^{(p r q)}(\{p, q, r\}, ш)-\mathcal{T}_{1 p 2} \frac{\left(k_{1} f_{p} X_{p}\right)}{\mathcal{K}_{1 p}} \mathcal{T}_{p r 2} \frac{\left(k_{1} f_{r} X_{r}\right)}{\mathcal{K}_{1 r}} \mathcal{T}_{r q 2} \frac{\left(k_{1} f_{q} X_{q}\right)}{\mathcal{K}_{1 q}}\right. \\
& \left.-\frac{\left[k_{p}\left(Y_{p}-k_{1}\right)\right]\left(k_{r} X_{r}\right)}{\mathcal{K}_{1 p r}} \mathcal{T}_{r q 2} \frac{\left(k_{1} f_{q} X_{q}\right)}{\mathcal{K}_{1 q}} \mathcal{T}_{p r 2} \mathcal{T}_{1 p 2} \frac{\left(k_{1} f_{r} f_{p} k_{1}\right)}{\mathcal{K}_{1 r} \mathcal{K}_{1 p}}\right] \\
= & \mathcal{K}_{1 p}\left[\mathcal{C}^{(p r q)}(\{p, q, r\}, w)-\frac{\left[k_{p}\left(Y_{p}-k_{1}\right)\right]\left(k_{r} X_{r}\right)}{\mathcal{K}_{1 p} \mathcal{K}_{1 p r}}\right] \\
= & \mathcal{K}_{1 p}\left[-\frac{\left[k_{p}\left(Y_{p}-k_{1}\right)\right]\left(k_{r} X_{r}\right)\left[k_{1}\left(Y_{q}-k_{1}\right)+\mathcal{K}_{1 p q r}\right]}{\mathcal{K}_{1 p} \mathcal{K}_{1 p r} \mathcal{K}_{1 p q r}}+\frac{\left[k_{p}\left(Y_{p}-k_{1}\right)\right]\left(k_{r} X_{r}\right)}{\mathcal{K}_{1 p} \mathcal{K}_{1 p r}}\right] \\
= & -\frac{\left[k_{p}\left(Y_{p}-k_{1}\right)\right]\left(k_{r} X_{r}\right)\left[k_{1}\left(Y_{q}-k_{1}\right)\right]}{\mathcal{K}_{1 p r} \mathcal{K}_{1 p q r}} .
\end{aligned}
$$


(3) $a(\{q, p, r\})$ : We have

$$
\begin{aligned}
a(\{q, p, r\})= & \mathcal{K}_{1 q}\left[\mathcal{C}^{(q p r)}(\{p, q, r\}, ш)-\mathcal{T}_{1 q p r 2} C_{0}(\{p, q, r\}, ш)\right] \\
= & \mathcal{K}_{1 q}\left[\mathcal{C}^{(q p r)}(\{p, q, r\}, ш)-\mathcal{T}_{p r 2} \frac{\left(k_{1} f_{r} X_{r}\right)}{\mathcal{K}_{1 r}} \mathcal{T}_{q p 2} \frac{\left(k_{1} f_{p} Y_{p}\right)}{\mathcal{K}_{1 p}} \mathcal{T}_{1 q 2} \frac{\left(k_{1} f_{q} X_{q}\right)}{\mathcal{K}_{1 q}}\right. \\
& \left.-\mathcal{T}_{p r 2} \frac{\left(k_{1} f_{r} X_{r}\right)}{\mathcal{K}_{1 r}} \mathcal{T}_{q p 2} \mathcal{T}_{1 q 2} \frac{\left(k_{1} f_{p} f_{q} k_{1}\right)}{\mathcal{K}_{1 p} \mathcal{K}_{1 q}} \frac{\left[k_{p}\left(Y_{p}-k_{1}\right)\right]\left(k_{q} X_{q}\right)}{\mathcal{K}_{1 p q}}\right] \\
= & \mathcal{K}_{1 q} \mathcal{C}^{(q p r)}(\{p, q, r\}, ш)=-\frac{\left(k_{q} X_{q}\right)\left[k_{p}\left(Y_{p}-k_{1}\right)\right]\left(k_{r} X_{r}\right)}{\mathcal{K}_{1 p q} \mathcal{K}_{1 p q r}} .
\end{aligned}
$$

(4) $a(\{q, r, p\}):$ We have

$$
\begin{aligned}
a(\{q, r, p\}) & =\mathcal{K}_{1 q}\left[\mathcal{C}^{(q r p)}(\{p, q, r\}, ш)-\mathcal{T}_{1 q r p 2} C_{0}(\{p, q, r\}, w)\right] \\
& =\mathcal{K}_{1 q}\left[-\frac{\left[k_{q}\left(X_{q}-k_{1}\right)\right]\left(k_{r} X_{r}\right)\left[k_{p}\left(Y_{p}-k_{1}\right)+\mathcal{K}_{1 p q r}\right]}{\mathcal{K}_{1 q} \mathcal{K}_{1 q r} \mathcal{K}_{1 p q r}}+\frac{\left[k_{q}\left(X_{q}-k_{1}\right)\right]\left(k_{r} X_{r}\right)}{\mathcal{K}_{1 q} \mathcal{K}_{1 q r}}\right] \\
& =-\frac{\left[k_{q}\left(X_{q}-k_{1}\right)\right]\left(k_{r} X_{r}\right)\left[k_{p}\left(Y_{p}-k_{1}\right)\right]}{\mathcal{K}_{1 q r} \mathcal{K}_{1 p q r}} .
\end{aligned}
$$

(5) $a(\{r, q, p\}):$ We have

$$
\begin{aligned}
a(\{r, q, p\}) & =\mathcal{K}_{1 r}\left[\mathcal{C}^{(r q p)}(\{p, q, r\}, w)-\mathcal{T}_{1 r q p 2} C_{0}(\{p, q, r\}, w)\right] \\
& =\mathcal{K}_{1 r}\left[-\frac{\left(k_{r} X_{r}\right)\left[k_{q}\left(X_{q}-k_{1}\right)+\mathcal{K}_{1 q r}\right]\left[k_{p}\left(Y_{p}-k_{1}\right)+\mathcal{K}_{1 p q r}\right]}{\mathcal{K}_{1 r} \mathcal{K}_{1 q r} \mathcal{K}_{1 p q r}}+\frac{\left(k_{r} X_{r}\right)\left[k_{q}\left(X_{q}-k_{1}\right)+\mathcal{K}_{1 q r}\right]}{\mathcal{K}_{1 r} \mathcal{K}_{1 q r}}\right] \\
& =-\frac{\left(k_{r} X_{r}\right)\left[k_{q}\left(X_{q}-k_{1}\right)+\mathcal{K}_{1 q r}\right]\left[k_{p}\left(Y_{p}-k_{1}\right)\right]}{\mathcal{K}_{1 q r} \mathcal{K}_{1 p q r}} .
\end{aligned}
$$

(6) $a(\{r, p, q\}):$ We have

$$
\begin{aligned}
a(\{r, p, q\}) & =\mathcal{K}_{1 r}\left[\mathcal{C}^{(r p q)}(\{p, q, r\}, ш)-\mathcal{T}_{1 r p q 2} C_{0}(\{p, q, r\}, ш)\right] \\
& =\mathcal{K}_{1 r} \mathcal{C}^{(r p q)}(\{p, q, r\}, ш)=-\frac{\left(k_{r} X_{r}\right)\left[k_{p}\left(Y_{p}-k_{1}\right)\right]\left[k_{q}\left(X_{q}+k_{r}\right)\right]}{\mathcal{K}_{1 p r} \mathcal{K}_{1 p q r}}
\end{aligned}
$$

In all the above calculations, we have used the results (2.21), which are the expansion of color ordered YM amplitudes to its BCJ basis.

[1] E. Witten, Commun. Math. Phys. 252, 189 (2004).

[2] F. Cachazo, S. He, and E. Y. Yuan, Phys. Rev. D 90, 065001 (2014).

[3] F. Cachazo, S. He, and E. Y. Yuan, Phys. Rev. Lett. 113, 171601 (2014).

[4] F. Cachazo, S. He, and E. Y. Yuan, J. High Energy Phys. 07 (2014) 033.

[5] F. Cachazo, S. He, and E. Y. Yuan, J. High Energy Phys. 01 (2015) 121.

[6] F. Cachazo, S. He, and E. Y. Yuan, J. High Energy Phys. 07 (2015) 149.
[7] S. Weinberg, Phys. Rev. 140, B516 (1965).

[8] F. Cachazo and A. Strominger, arXiv:1404.4091.

[9] E. Casali, J. High Energy Phys. 08 (2014) 077.

[10] B. U. W. Schwab and A. Volovich, Phys. Rev. Lett. 113, 101601 (2014).

[11] N. Afkhami-Jeddi, arXiv:1405.3533.

[12] M. Zlotnikov, J. High Energy Phys. 10 (2014) 148.

[13] C. Kalousios and F. Rojas, J. High Energy Phys. 01 (2015) 107.

[14] F. Cachazo, S. He, and E. Y. Yuan, Phys. Rev. D 92, 065030 (2015). 
[15] Z. Bern, J. J. M. Carrasco, and H. Johansson, Phys. Rev. D 78, 085011 (2008).

[16] Z. Bern, J. J. M. Carrasco, and H. Johansson, Phys. Rev. Lett. 105, 061602 (2010).

[17] H. Kawai, D. C. Lewellen, and S. H. H. Tye, Nucl. Phys. B269, 1 (1986).

[18] Z. Bern, J. J. Carrasco, M. Chiodaroli, H. Johansson, and R. Roiban, arXiv:1909.01358.

[19] C. H. Fu, Y. J. Du, R. Huang, and B. Feng, J. High Energy Phys. 09 (2017) 021.

[20] B. Feng, S. He, R. Huang, and Y. Jia, J. High Energy Phys. 10 (2010) 109.

[21] S. Stieberger and T. R. Taylor, Nucl. Phys. B913, 151 (2016).

[22] O. Schlotterer, J. High Energy Phys. 11 (2016) 074.

[23] M. Chiodaroli, M. Gunaydin, H. Johansson, and R. Roiban, J. High Energy Phys. 07 (2017) 002.

[24] V. Del Duca, L. J. Dixon, and F. Maltoni, Nucl. Phys. B571, 51 (2000).

[25] D. Nandan, J. Plefka, O. Schlotterer, and C. Wen, J. High Energy Phys. 10 (2016) 070.

[26] L. de la Cruz, A. Kniss, and S. Weinzierl, Phys. Lett. B 767, 86 (2017).

[27] C. Cardona, B. Feng, H. Gomez, and R. Huang, J. High Energy Phys. 09 (2016) 133.

[28] N. E. J. Bjerrum-Bohr, J. L. Bourjaily, P. H. Damgaard, and B. Feng, Nucl. Phys. B913, 964 (2016).

[29] N. E. J. Bjerrum-Bohr, J. L. Bourjaily, P. H. Damgaard, and B. Feng, J. High Energy Phys. 09 (2016) 094.

[30] R. Huang, Y. J. Du, and B. Feng, J. High Energy Phys. 06 (2017) 133.

[31] F. Teng and B. Feng, J. High Energy Phys. 05 (2017) 075.

[32] Y. J. Du and F. Teng, J. High Energy Phys. 04 (2017) 033.

[33] Y. J. Du, B. Feng, and F. Teng, J. High Energy Phys. 12 (2017) 038.
[34] C. Cheung, C. H. Shen, and C. Wen, J. High Energy Phys. 02 (2018) 095.

[35] K. Zhou and B. Feng, J. High Energy Phys. 09 (2018) 160.

[36] M. Bollmann and L. Ferro, J. High Energy Phys. 01 (2019) 180.

[37] B. Feng, R. Huang, and Y. Jia, Phys. Lett. B 695, 350 (2011).

[38] Y. X. Chen, Y. J. Du, and B. Feng, J. High Energy Phys. 02 (2011) 112.

[39] N. E. J. Bjerrum-Bohr, P. H. Damgaard, and P. Vanhove, Phys. Rev. Lett. 103, 161602 (2009).

[40] S. Stieberger, arXiv:0907.2211.

[41] R. Kleiss and H. Kuijf, Nucl. Phys. B312, 616 (1989).

[42] L. Hou and Y. J. Du, J. High Energy Phys. 05 (2019) 012.

[43] C. S. Lam and Y.P. Yao, Phys. Rev. D 93, 105008 (2016).

[44] S. He and Y. Zhang, J. High Energy Phys. 02 (2017) 019.

[45] Z. Bern, L. J. Dixon, D. C. Dunbar, M. Perelstein, and J. S. Rozowsky, Nucl. Phys. B530, 401 (1998).

[46] N. E. J. Bjerrum-Bohr, P. H. Damgaard, B. Feng, and T. Sondergaard, Phys. Rev. D 82, 107702 (2010).

[47] N. E. J. Bjerrum-Bohr, P. H. Damgaard, B. Feng, and T. Sondergaard, Phys. Lett. B 691, 268 (2010).

[48] N. E. J. Bjerrum-Bohr, P. H. Damgaard, B. Feng, and T. Sondergaard, J. High Energy Phys. 09 (2010) 067.

[49] R. H. Boels and H. Luo, J. High Energy Phys. 05 (2018) 063.

[50] L. A. Barreiro and R. Medina, Nucl. Phys. B886, 870 (2014).

[51] R. H. Boels and R. Medina, Phys. Rev. Lett. 118, 061602 (2017).

[52] N. Arkani-Hamed, L. Rodina, and J. Trnka, Phys. Rev. Lett. 120, 231602 (2018).

[53] Z. Bern, A. Edison, D. Kosower, and J. Parra-Martinez, Phys. Rev. D 96, 066004 (2017). 